Illinois State University

ISU ReD: Research and eData

Theses and Dissertations

$1-30-2018$

\title{
Young Adults with Visual Impairments and Driver's Education: Journeys of Self-Efficacy, Identity, and Transition to Adulthood
}

Molly Pasley

Illinois State University, mollyclesen@gmail.com

Follow this and additional works at: https://ir.library.illinoisstate.edu/etd

Part of the Disability Studies Commons, Special Education Administration Commons, and the Special Education and Teaching Commons

\section{Recommended Citation}

Pasley, Molly, "Young Adults with Visual Impairments and Driver's Education: Journeys of Self-Efficacy, Identity, and Transition to Adulthood" (2018). Theses and Dissertations. 1032.

https://ir.library.illinoisstate.edu/etd/1032

This Dissertation is brought to you for free and open access by ISU ReD: Research and eData. It has been accepted for inclusion in Theses and Dissertations by an authorized administrator of ISU ReD: Research and eData. For more information, please contact ISUReD@ilstu.edu. 


\title{
YOUNG ADULTS WITH VISUAL IMPAIRMENTS AND DRIVER'S EDUCATION: JOURNEYS OF SELF-EFFICACY, IDENTITY, AND TRANSITION TO ADULTHOOD
}

\author{
Molly Pasley
}

\section{Pages}

In this qualitative study, young adults with visual impairments aged 18-27 and family members were studied to determine the effects of state-mandated, high school driver's education on independent travel, self-efficacy, and transition to adulthood. A young person's ability to travel independently can determine whether he or she transitions successfully from one life stage to another. Kim's (2015) concept of “unbecoming human” and HeavyRunner \& Marshall's (2003) concept of resilience form the theoretical frame for data analysis. The author finds that in spite of curricular and pedagogical intervention, regardless of technological advances, and despite low-vision/blind youth's accomplishments and determination to succeed, young people with low vision/blindness nevertheless continue to be challenged in the realms of transition to adulthood, securing employment, and independent living due to persistent, societal-level discrimination and perceived incapability. These persistent, unwarranted forms of discrimination profoundly affect young adults' perceptions of self-efficacy and more often than not close doors to their success. The author posits societal-level intervention such as that proposed by Kim is required to affect meaningful, equitable change designed to dismantle the current, systemic discrimination plaguing youth and young adults with visual impairments. KEYWORDS: visual impairments; nondriving youth; independent travel; driver's education; transition; resilience 
YOUNG ADULTS WITH VISUAL IMPAIRMENTS AND DRIVER'S EDUCATION:

JOURNEYS OF SELF-EFFICACY, IDENTITY, AND TRANSITION TO ADULTHOOD

\author{
MOLLY PASLEY
}

A Dissertation Submitted in Partial

Fulfillment of the Requirements for the Degree of

DOCTOR OF EDUCATION

Department of Special Education

ILLINOIS STATE UNIVERSITY 
Copyright 2019 Molly Pasley 
YOUNG ADULTS WITH VISUAL IMPAIRMENTS AND DRIVER'S EDUCATION:

JOURNEYS OF SELF-EFFICACY, IDENTITY, AND TRANSITION TO ADULTHOOD

MOLLY PASLEY

COMMITTEE MEMBERS:

Olaya Landa-Vialard, Co-Chair

Stacy Otto, Co-Chair

April Mustian

Debbie Shelden 


\section{ACKNOWLEDGMENTS}

I first want to thank my husband for being there during this long journey. I would then like to thank my dissertation co-chairs for their guidance, support, and directness. Their positivity and kindness kept me moving. I also want to thank the remaining two members of the dissertation committee for their candid feedback. All critiques helped to make this a richer final product.

M.A.P. 


\section{CONTENTS}

\section{Page}

ACKNOWLEDGMENTS

CONTENTS

TABLES

CHAPTER I: INTRODUCTION 1

Statement of the Problem 1

$\begin{array}{ll}\text { Statement of Purpose } & 2\end{array}$

$\begin{array}{ll}\text { Research Question } & 3\end{array}$

Research Context $\quad 3$

Definition of Identity 3

Identity construction for people with disabilities $\quad 4$

Quality of Life, Transition, and Factors Influencing Independence $\quad 7$

$\begin{array}{ll}\text { Position of Study in the Literature } & 8\end{array}$

CHAPTER II: REVIEW OF THE LITERATURE 10

The Evolution of Education for People with Low Vision/Blindness 11

Educational Settings $\quad 11$

$\begin{array}{ll}\text { Residential schools } & 12\end{array}$

$\begin{array}{ll}\text { Inclusion in public schools } & 14\end{array}$

Rehabilitation centers for the visually impaired $\quad 15$

Quality of Life 16

Quality of Life for Different Disability Groups 17

Quality of Life for People with Visual Impairments 18 
Operational Definition of Quality of Life

$\begin{array}{ll}\text { Secondary Transition } & 20\end{array}$

$\begin{array}{ll}\text { Transition Definition and History } & 20\end{array}$

$\begin{array}{ll}\text { Independence } & 23\end{array}$

Transition Experiences of Individuals with Visual Impairments 27

Expanded Core Curriculum (as a vehicle for secondary transition for those with VI) 30

Orientation and mobility $\quad 30$

Self-determination 33

Independence of Travel 36

$\begin{array}{ll}\text { Factors Affecting Independence } & 36\end{array}$

$\begin{array}{ll}\text { Disability characteristics } & 36\end{array}$

$\begin{array}{ll}\text { Learned helplessness } & 38\end{array}$

$\begin{array}{ll}\text { Culture } & 40\end{array}$

$\begin{array}{ll}\text { Family } & 42\end{array}$

$\begin{array}{ll}\text { Driver's Education } & 44\end{array}$

$\begin{array}{ll}\text { Drivers and Nondrivers with Disabilities } & 44\end{array}$

Effect of Nondriving on Quality of Life 45

$\begin{array}{ll}\text { CHAPTER III: METHODOLOGY } & 48\end{array}$

$\begin{array}{ll}\text { Theoretical Framework } & 48\end{array}$

$\begin{array}{lr}\text { Biases and Subjectivities } & 49\end{array}$

$\begin{array}{ll}\text { Population } & 52\end{array}$

$\begin{array}{ll}\text { Methodology } & 54\end{array}$

$\begin{array}{ll}\text { Methods } & 56\end{array}$ 
$\begin{array}{ll}\text { Methods of Analysis } & 58\end{array}$

$\begin{array}{ll}\text { Theoretical Framework for Data Analysis } & 58\end{array}$

Theoretical Concepts Defined $\quad 59$

$\begin{array}{ll}\text { History of resilience theory } & 61\end{array}$

$\begin{array}{ll}\text { Definition of resilience } & 62\end{array}$

$\begin{array}{ll}\text { Unbecoming human } & 64\end{array}$

Methodological Roots and Formative-Analysis-Based Design Changes 67

$\begin{array}{ll}\text { CHAPTER IV: DATA ANALYSIS } & 77\end{array}$

$\begin{array}{ll}\text { Participant Descriptions } & 77\end{array}$

$\begin{array}{ll}\text { Irene } & 77\end{array}$

$\begin{array}{lr}\text { Maverick } & 80\end{array}$

$\begin{array}{ll}\text { Nolan } & 83\end{array}$

$\begin{array}{ll}\text { Devon } & 85\end{array}$

$\begin{array}{ll}\text { Amelia } & 87\end{array}$

$\begin{array}{ll}\text { Tommy } & 91\end{array}$

$\begin{array}{ll}\text { Wolfgang } & 94\end{array}$

Societal Perception of Humanity of People with Visual Impairments 97

Societal Perception of Humanity and Identity Construction 105

Societal Perception of Humanity and Driver's Education 115

Societal Perception of Humanity Based on Nondriving $\quad 120$

Overcoming Societal Perception of Humanity to Construct Healthy Identities 124

CHAPTER V: FINDINGS, IMPLICATIONS, \& RECOMMENDATIONS 132

Findings and Implications 133 
Perceptions of Incapacity

$\begin{array}{ll}\text { Driving as a Metaphor for Ableism } & 135\end{array}$

$\begin{array}{ll}\text { School Pushout } & 136\end{array}$

$\begin{array}{ll}\text { Recommendations for Practice } & 139\end{array}$

$\begin{array}{ll}\text { TVIs and COMS in PK-12 Schools } & 140\end{array}$

Researchers of People with Visual Impairments $\quad 142$

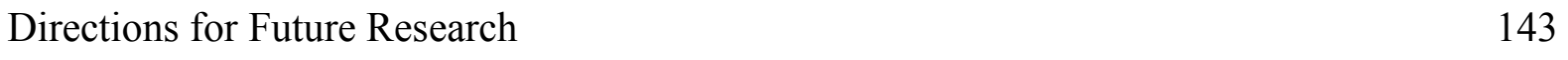

$\begin{array}{ll}\text { For Practitioners } & 143\end{array}$

$\begin{array}{ll}\text { Application of Social Theory } & 144\end{array}$

$\begin{array}{ll}\text { REFERENCES } & 147\end{array}$

APPENDIX A: LEVEL ONE SOLICITATION EMAIL (TO DIRECTORS OF

UNIVERSITY/COMMUNITY COLLEGE DISABILITY SERVICES) 160

$\begin{array}{ll}\text { APPENDIX B: PARTICIPANT RECRUITMENT EMAIL } & 161\end{array}$

APPENDIX C: PHONE SCRIPT WITH INCLUSIONARY QUESTIONS TO DETERMINE

$\begin{array}{lc}\text { PARTICIPANT ELIGIBILITY } & 163\end{array}$

APPENDIX D: FAMILY MEMBER RECRUITMENT SCRIPT 165

$\begin{array}{ll}\text { APPENDIX E: FAMILY MEMBER RECRUITMENT EMAIL } & 168\end{array}$

APPENDIX F: CONSENT LETTER FOR ADULT PARTICIPANTS WITH VISUAL

$\begin{array}{lr}\text { IMPAIRMENTS } & 170\end{array}$

APPENDIX G: CONSENT LETTER FOR FAMILY MEMBER OF PERSON WITH VISUAL

$\begin{array}{ll}\text { IMPAIRMENTS } & 173\end{array}$

$\begin{array}{ll}\text { APPENDIX H: PARTICIPANT INTERVIEW PROTOCOL } & 176\end{array}$

APPENDIX I: FAMILY MEMBER INTERVIEW PROTOCOL 178 


\section{TABLES}

Table

Page

1. Erikson's Stages of Psychosocial Development 


\section{CHAPTER I: INTRODUCTION}

A person's ability to travel independently can determine whether he or she transitions successfully from one life stage to another. One critically important aspect of independent travel is coming to understand how vehicles move in the built environment. For youth without visual disabilities, this information is addressed in depth in driver's education courses. In the case of adolescents with visual impairments, the acquisition of this information raises a number of complicated questions. Should students who will never be eligible to drive be required to take part in a class the result of which they will never achieve the same level of independence, freedom, and responsibility as their sighted peers? Is the material in the course and the manner in which it is taught meaningful enough to supersede potentially negative feelings experienced by partaking in such a class?

\section{Statement of the Problem}

In an age of "helicopter parenting," it can be challenging for youth with visual impairments to establish and realize independence, negatively affecting identity construction. The helicopter parenting phenomena is even more enhanced for children with visual impairments. By the nature of their disability, people with visual impairments are dependent upon others to access the sighted world. As a result, it is even more critical for those with low vision/blindness (LV/B) to acquire the skills to demonstrate their independence if they are to develop a positive quality of life. A reduction in independence has the potential for lasting effects on the ability of people with LV/B to acquire and demonstrate the skills necessary to travel independently and obtain gainful employment, significantly affecting transition from school to adulthood. 


\section{Statement of Purpose}

There are certain expectations of how a person exhibits independence. One of the ways in which people without disabilities demonstrate their independence is through acquisition of a driver's license. The issue of driver's education for people with visual impairments was brought to my attention as a second-year teacher of students with visual impairments (TVI) working for a rural, special-education cooperative. In one of the member high schools, I worked with a student with low vision who was required to participate in driver's education in order to graduate. This particular student felt strongly he should not have to take driver's education because he did not meet his state's vision requirement to obtain a driver's license. After consulting with administration, it was determined that he would not take driver's education but would replace the credit earned by taking a different class. As a budding mobility specialist and newly graduated TVI, this experience led me to research available alternatives to driver's education for students with visual impairments. During this search, I was able to locate two curricula designed to assist people with visual impairments learn to utilize various forms of transportation: Finding Wheels (Rosenblum \& Corn, 2000) and Going Places (Rosenblum, 2004). The former is designed specifically for instructing adolescents with visual impairments while the latter is written to address the unique needs of adults who lose vision after years of driving. Access to these curricula, however, is based on an instructor's awareness of their existence. Due to lack of awareness of Finding Wheels, in particular, it is not widely used by COMS working with adolescents with visual impairments (Sacks \& Rosenblum, 2006; personal communication, Feb $15,2018)$. 


\section{Research Question}

While the perceptions of driving and nondriving have been researched, currently there is no available literature that examines the perceived value of driver's education to nondrivers with visual impairments from any age group. Specifically, I pose the question:

What are the perceptions of the impact of driver's education on nondriving young adults with visual impairments?

The original research question above evolved during data collection, organization and analysis and I detail and justify the study's evolution in Chapter 3.

\section{Research Context}

Development of a healthy identity is important in the transition from student to adult. Identity is tied to self-efficacy, desires for independence, and quality of life. Promoting construction of positive identities through education is one of the goals of professionals who work with people with visual impairments. In the following, I define identity and how identities are constructed by people with disabilities. I then showcase the ways in which identity is constructed by addressing a number of overarching themes that have the potential to positively or negatively affect how a person views himself and the ways in which a person's identity may affect his independence, quality of life, and subsequent transition from post-school to adulthood.

\section{Definition of Identity}

Erikson's (1950) seminal work on identity in terms of developmental stages that occur from birth through adulthood forms the basis of many derivatives of the term as we know it today. According to Erikson (1950), a person's identity is formed by working through dichotomous psychosocial states that build on one another. The foundational stages of development that take place between birth to three years of age include trust/mistrust and 
autonomy/shame and doubt. When children with visual impairments reach school age, they have the potential to progress through initiative/guilt, industry/inferiority, and identity/role confusion. During these stages are when youth and adolescents are at their greatest risk to construct unhealthy identities, especially if they are lacking support and positive experiences related to life with a visual impairment. Also, perception of their inability to engage in some of these maturational processes (e.g. driving) may have a negative influence on identity construction.

While Erikson's stages of development mark the starting point in a long line of researchers who seek to define identity in a variety of contexts (Helms, 1997; Kelly \& Millward, 2002; Marcia, 1966, 1980, 2002), they are limited in their discrete bipolarity. For this study, I look to a more complex definition of identity that highlights the social aspects of identity and accounts for intersectionality of variables in place of a dichotomy of opposites. Fearon (1999) defines identity as either a social category governed by membership rules and expected behaviors, or a feature that distinguishes someone socially that she takes special pride in, or a combination of the two. This definition is most appropriate to the phenomenon under investigation because it is representative of the ways in which young adults with visual impairments construct their identities; through membership to a specific social category (e.g. smart, independent traveler) and/or a distinguishing feature someone takes special pride in (e.g. independent travel, accommodation for vision loss, advocation). It also focuses attention on the social aspects of identity construction, highlighting the external and collective factors that comprise identity, which also relates to the collective nature of resilience theory (HeavyRunner \& Marshall, 2003).

Identity construction for people with disabilities. There are multiple dimensions at play in the construction of the individual sense of self. These include but are not limited to: race, 
ethnicity, sexuality, gender, and disability. In the past two decades, research has focused more on the intersectionality of the multiple dimensions in place of a singular category to describe identity (Jones \& McEwen, 2000). This is in large part because people are not shaped by just one social group to which they subscribe. A black woman who is deaf is going to have different experiences that form her identity not just as a member of the black community, but also as a woman who belongs to the Deaf community. To attempt to isolate one "variable" in the development of a person's identity is to neglect the richness of it in its entirety and to ignore the multiple ways in which a person who is a member of multiple marginalized groups may be othered and the way layered identities increase exponentially the ways individuals belong and clash with their world.

Forber-Pratt and Zape (2017) build on previous research concerning identity development for people with disabilities in their qualitative study to establish a "psychosocial identity development model" (p. 350) that seeks to explain how college students with disabilities develop their identities. College students with varying disabilities were selected because for the majority, if not all, of their lives, the Americans with Disabilities Act has been in existence. Forber-Pratt and Zape (2017) wanted to see if growing up in a more inclusive culture (as a result of legislation) that sought to eliminate discrimination against people with disabilities shifted how people with disabilities perceived their identity. Results indicate that internal and external factors play a role in identity development to create a model that "highlights four developmental statuses: acceptance, relationship, adoption and engagement" (p. 350). This research is important because it provides educators with a greater understanding of the psychological processes that people with disabilities experience so we can provide experiences for youth and young adults to construct healthy identities, which contributes to a stronger sense of self and by reaffirming 
desired goals and personal worth, individuals are better equipped to face ableism (Albrecht \& Devlieger, 1999; Campbell, 2008; Mpofu \& Harley, 2006; Noonan et al., 2004).

People rise or fall to meet the expectations of others. Consider the additional challenge placed on a person who has been branded as "dis"abled. Not only does his condition require him to learn in a way that is outside the mainstream expectation of "typical," he continuously pushes against an ableism mentality. Labels can be informative but they can also be detrimental to a person's identity. Take for example the terms "at-risk" and "achievement gap." These terms are based in deficit-thinking and are based largely on race and socio-economic status (Valencia, 2010). Labeling a student as "at-risk" or the existence of an "achievement gap" implies there is an environmental deficit that puts some people at a higher risk of failing out of secondary school. Instead of highlighting the systemic problems that make a school a hostile environment, the focus is placed on the student, his family, and his cultural "differences" (Valencia, 2010). Once these labels are acquired they cannot be escaped and become part of a child's identity. Many parents are reluctant to diagnose or label a child as having a disability because of the stigmas carried by certain disability categorizations. Once a diagnosis is prescribed, many families look to finding a "cure," whether viable or not, for the cause of the disability because having a disability is viewed as a problem that requires fixing. This has implications for the individual with the disability because this identity as "broken" results in tireless attempts to prove something, become something else, or thrive "in spite of" a perceived disadvantage. These experiences are especially true for people with visual impairments because vision is the main channel through which we acquire information. Any dysfunction in this ability is greater amplified as a result and has the potential to negatively affect self-efficacy, independence, quality of life, and transition to adulthood. 


\section{Quality of Life, Transition, and Factors Influencing Independence}

The history of educating people with visual impairments is rich and well documented. Through the development of residential schools for the visually impaired and inclusion in public schools, children with visual impairments have access to an education that is vastly different than what they experienced over a century ago. Presently, the focus is on producing self-determined young adults who are able to achieve a positive quality of life through successful transition from secondary school.

Quality of life research concerning people with various types of impairment is extensive. So much so that the eight-domain human-rights-oriented model of quality of life has been validated and demonstrates universality across disability area and cross-culturally (Jenaro et al., 2005; Schalock et al., 2005; Wang, Schalock, Verdugo, \& Jenaro, 2010). In Sheffield's (2014) exploratory study, she adapts Schalock and Verdugo's (2002) validated instrument to measure quality of life for people who are blind. Through her work, she identifies "independence," specifically self-determination, self-reliance, and self-advocacy as measures of quality of life for individuals with total blindness. Based on Sheffield's findings, I investigate further the research regarding the role of independence in post-school transition outcomes through analyses of the National Longitudinal Transition Study and National Longitudinal Transition Study 2 for all students with disabilities and whose subjects are limited to students with visual impairments.

The results of my investigation of the aforementioned themes led me to distill two areas of the expanded core curriculum that are important to independence and positive transition outcomes for youth with visual impairments: orientation and mobility (O\&M) and selfdetermination. Sheffield (2014) notes the value of self-determination in realizing independence and Wolffe and Kelly (2011) list O\&M as one of the indicators for successful transition among 
adolescents with LV/B. With the importance placed on independence from a number of disciplines and how it is involved in people living their best lives by achieving positive quality of life and transition outcomes, I look at some of the factors that can affect independence. People's perceptions based on disability characteristics, learned helplessness, culture, and family all have the potential to inhibit and/or promote independence in life and in travel. One of the ways in which people demonstrate their independence, freedom, and responsibility is by acquiring a driver's license.

In the U.S., learning to drive has long been viewed as a sign of independence among teens and young adults (Corn \& Rosenblum, 2000; Sacks \& Rosenblum, 2006; Schlecter \& Gump, 1983). The same is true for adolescents with visual impairments. For older adults who stop driving due to vision loss the change leaves individuals feeling reduced self-control and independence (Eisenhelder, 1990), because the driver's license can serve as a "symbol of selfworth" (Meier, 1992, p. 17). I close my review of the literature by examining the research concerning drivers and nondrivers with disabilities and the effect of nondriving on perceived independence and quality of life.

\section{Position of Study in the Literature}

In my research study, I investigate the perceived value of participation in driver's education by people with LV/B by interviewing young adults with visual impairments aged 1827 and their family members. I also observed participants with visual impairments engaged in independent travel activities (e.g. walking to class, while at work, riding public transportation). Participants indicate that there is much more involved in the issue of nondriving than simply satisfying a graduation requirement by participating in a driver's education course. Their experiences in driver's education, during independent travel, and in educational/work settings 
have lasting effects on their self-efficacy, identity construction, and transition to adulthood. In addition, participants describe what I consider to be a societal perception of incapacity by some based on their visual impairment and inability to drive. The findings of this study are significant because participants expose instances of pervasive discrimination, despite the existence of legislation that seeks to include people with disabilities, and how these attitudes create a limit on what a person can achieve, effectively eliminating upward movement. Perceptions of incapacity also have the potential to increase learned helplessness, which is counterproductive when attempting to realize successful transition from school to adult life. The implications of this study are alarming. Discouragingly, teaching people with visual impairments how to accommodate, alone, is not enough to achieve equity because it does not challenge societal norms and discrimination. Analysis of the data and participants' descriptions of their experiences with discrimination leads me to conclude that professionals working with people with visual impairments need to be educating activists in addition to creating highly competent and highly capable individuals. 


\section{CHAPTER II: REVIEW OF THE LITERATURE}

Because this phenomenon gives rise to a number of questions, in this study I set out to illuminate the perceived value of driver's education among young people with visual impairments. I first couch my proposed dissertation study in the research literature to identify the gap in the literature my study fills. Before laying out the sociocultural and educational aspects of this phenomenon, I initially provide my readers with an historical context through which the evolving education of people with visual impairments can be brought into perspective. I then overlay the history of educating this unique population with the sociocultural, presenting research and theory concerning the quality of life of individuals with disabilities. In this section I also recount the range of definitions of quality of life, consolidating the range into one operational definition I will employ in my study. Since research has long established successful transition to have a positive effect on quality of life, I also unpack the concept of "transition," providing the reader with a definition and history of the term, describing transition experiences of people with various disabilities, and explaining how the expanded core curriculum (ECC) serves as the vehicle by which youth with visual impairments receive secondary transition training. I close by laying out research on experiences of people with visual impairments, including factors affecting independence of travel, experiences of nondrivers with different disabilities, and the effect the ability (or inability) to drive has on the quality of life of a person with a visual impairment. These seemingly disparate areas of research and theory intersect because, perhaps surprisingly, although the ability to drive is just as important and as significant a maturational milestone to adolescents with visual impairments as it is to their sighted peers (Corn \& Sacks, 1994; Peterson, 1992), driver's education options available in public schools are 
limited. Through my literature review, I work to expose a gap in the research regarding driver's education and students with visual impairments that my research question is designed to address: What are the perceptions of the impact of driver's education on nondriving young adults with visual impairments?

\section{The Evolution of Education for People with Low Vision/Blindness}

Since the creation of the first residential school for the blind by Samuel Gridley Howe in Boston in 1829 (Hatlen, 2000), the education of people with visual impairments has evolved greatly. Instead of the exception, inclusion has become the norm. Although many students are now served in public day schools by professionals trained to work with children with visual impairments, residential schools and rehabilitation centers continue to play a key role in preparing children and adults with visual impairments to enter and reenter the workforce (Hatlen, 2000). The three placements available to people with visual impairments are residential schools, inclusion in public schools, and rehabilitation centers.

\section{Educational Settings}

Residential schools, public schools, and rehabilitation centers for people with vision loss are all settings where the focus of instruction is to make the individual more independent. This is so people with visual impairments learn to make decisions and contribute to their family and society in any way appropriate to their situation and abilities. The International Association for the Scientific Study of Intellectual Disability (IASSID) recommends a human-rights-oriented, eight-domain model to measure a person's quality of life (Schalock \& Verdugo, 2002). While this model is not specific to individuals with visual impairments, instruction that occurs in the aforementioned educational settings, specifically, independence and independent travel are directly related to five of the eight domains including emotional well-being, personal 
development, social inclusion, self-determination, and rights (Schalock \& Verdugo, 2002). One cannot truly understand the education of people with LV/B without first looking back at how it began. To this end, I recount the evolution of residential schools, inclusion of children with visual impairments in public schools, and rehabilitation centers for people who are visually impaired.

Residential schools. Public perception of people with disabilities has had a significant effect on the self-perception of people with visual impairments. For the last century, public expectations of people with disabilities have been low (Monbeck, 1973; Welsh, 1997). Prior to 1900, there was little support or encouragement for people who were blind, causing this population to resort to performing public skits that elicited ridicule and pity from the public, and often being reduced to begging to earn money (Hatlen, 2000). Samuel Gridley Howe held very different beliefs from the general public regarding the aptitudes of the blind. He maintained every child with a visual impairment must be considered an individual and be educated according to his or her interests and abilities, that curricula should conform as closely as possible to that of public day schools, and that students must be prepared to take their place in the social and economic lives of their communities (Hatlen, 2000; Lowenfeld, 1973). Howe's attitude continues to be the driving force in personnel preparation programs for professionals working with people with visual impairments, whose professional purpose is to "work ourselves out of a job," the ultimate goal being to help people develop the skills to participate socially and economically in the marketplace, demonstrating their independence. During a time of institutionalization of people with disabilities, the creation of residential schools in the United States was an effort to empower this population, not repress it (Hatlen, 2000). The development of residential schools was precipitated by geographic necessity and the low prevalence of 
blindness in children, the combination of which ultimately revolutionized instruction of students with low vision/blindness.

From 1829 until the 1950s, many students with visual impairments attending residential schools were educated the same, regardless of functional vision (Hatlen, 2000). Many students with low vision were taught as though they were blind and were made eligible in residential schools and public schools using the legal definition of blindness (i.e., central visual acuity of $20 / 200$ or less in the better eye with best correction or a visual field loss of 20 degrees or less; Koestler, 1976), "primarily using tactile or auditory senses for learning" (Hatlen, 2000, p. 16). In 1879, the American Printing House for the Blind (APH) was established to produce instructional materials for students with visual impairments. Braille, large print textbooks, and various other materials were made available to students with visual impairments by the provision of federal quota funds.

During the 1930s, there was a great exodus of students with visual impairments from residential settings to the secondary schools in their communities. Superintendents of some residential schools believed students were ready to compete with their sighted peers academically, and oftentimes, schools for the blind only offered limited academic curricula due to the small number of attendees (Hatlen, 2000). In the 1950s and 1960s, the role of residential schools shifted to include students with additional disabilities because students with visual impairments were educated in their home schools. As a result, some schools added state-wide assessment services, technical assistance to local school districts, and professional development for teachers of the visually impaired (TVIs; Hatlen, 2000). In addition, some coordinated outreach and acted as hubs for services (Hatlen, 2000). Residential schools for the visually impaired continue to provide assessment services, technical assistance when necessary, and 
professional development for TVIs. One example of a school that continues to provide resources to professionals and students with visual impairments is the Texas School for the Blind and Visually Impaired. The staff have created braille reading curricula and supply a vast amount of math braille resources on their website. Residential schools opened the door for educating children with visual impairments and was the first step in what Howe hoped would turn into educating children with low vision and blindness in public day schools.

Inclusion in public schools. Parental advocacy and the belief students with visual impairments could contribute to the sighted world helped spark the movement toward an improved quality of life for future individuals with LV/B. The rise in the number of premature infants who were treated with large amounts of oxygen in infancy caused a sharp incline in the visually impaired population during the 1940s when premature infants were prescribed large amounts of oxygen, which caused scar tissue to form behind the eye's lens, resulting in retinopathy of prematurity (ROP; Hatlen, 2000). As children reached school age, schools were unable to accommodate the needs of a now-vast number of students with LV/B.

The surge in the ROP population had a significant effect on the public education of students with visual impairments. Many of these children came from middle- and upper-class families who were influential in their communities and did not want to ship their children away to residential schools (Hatlen, 2000). Since, for the first time, there were a large number of students concentrated in a single area, school districts could now justify hiring a TVI, which had previously only been possible for people living in major cities where the population was much denser (Hatlen, 2000).

The increased demand for trained teachers likely affected the number and quality of personnel preparation programs across the U.S. As the number of students and professionals 
increased, people with visual impairments could no longer be overlooked. Addressing many other disability areas, legislation was passed to provide guidance for further educating students with visual impairments (Education for All Handicapped Children Act of 1975, 2016; Individuals with Disabilities Education Act of 1990, 2016; Individuals with Disabilities Education Improvement Act of 2004, 2016).

Rehabilitation centers for the visually impaired. Rehabilitation centers, among public schools and residential schools, are designed to help foster independence and improve quality of life of people with visual impairments. The field of blind rehabilitation began as an offshoot of educating children with visual impairments (Ponchillia \& Ponchillia, 1996). Rehabilitation teaching first took place in the home of the person who had lost her vision. The teachers of these individuals were also blind. Over time, instructors realized that people needed more than just reading and writing skills; they needed to learn daily living skills as well to maintain their homes and care for themselves (Ponchillia \& Ponchillia, 1996).

Similar in the sense that a major event sparked the revolution in educational services for children with visual impairments, World War I prompted the establishment of residential rehabilitation centers. The return of a large number of disabled servicemen from World War I prompted the creation of the first rehabilitation center in the U.S. in 1918. The numbers of injured veterans continued to increase after World War II, leading to formal training of servicemen at blind rehabilitation centers in the Veterans Administration (Ponchillia \& Ponchillia, 1996). The growing number of blind rehabilitation programs prompted formal training programs for blind rehabilitation teachers. By 1950, 30 states operated residential rehabilitation centers (Ponchillia \& Ponchillia, 1996). 
Regardless of setting, the education of people with visual impairments continues to grow and develop. One of the driving instructional forces for this population is teaching individuals the skills required so they can engage with their environment as independently as appropriate. A person's ability to complete tasks independently makes him feel as though he has something to offer of value. A person's perceived value directly affects his emotional well-being, one of the measures of his quality of life.

\section{Quality of Life}

Many people measure their overall contentment by examining standards they set for perceived quality of life. Quality of life is a construct defined as "the extent to which a person's needs and desires are satisfied [or exceeded] by the circumstances in which they live" (Sheffield, 2014, p. 1). In the human rights-oriented model of quality of life recommended by the International Association for the Scientific Study of Intellectual Disability (IASSID; Schalock \& Verdugo, 2002), the focus is on eight domains: (a) emotional well-being, (b) interpersonal relationships, (c) material well-being, (d) personal development, (e) physical well-being, (f) social inclusion, (g) self-determination, and (h) rights (Schalock \& Verdugo, 2002; Sheffield, 2014). This model was originally designed using research data on people with intellectual and closely related developmental disabilities; however, it is relatable to the quality of life of people who are visually impaired, especially as nondrivers. One of the unique aspects of quality of life is it is measured differently by different groups. Next I examine research regarding quality of life for varying disability groups and use this information to construct an operational definition of quality of life for my study. 


\section{Quality of Life for Different Disability Groups}

Although groups measure quality of life differently, some commonalities do exist.

Components of the prevailing eight-domain human-rights model of quality of life have shown to be generalizable to mental health, chemical dependence, physical and sensory disabilities, and the elderly (Gomez, Peña, Arias, \& Verdugo, 2014). Additionally, researchers have demonstrated the universality of this model of quality of life cross-culturally (Jenaro et al., 2005; Schalock et al., 2005; Wang et al., 2010).

Schalock et al. (2005) conducted a quantitative study of the cross-cultural validity of the IASSID's eight-domain model. In their survey, three respondent groups from five geographical groupings responded to the importance and use of 24 core quality of life indicators most commonly found in the international quality of life research. Results indicate that while some indicators of the IASSID's quality of life model are universal and traverse cultural lines, some are culturally bound to the context within people live. The authors suggest that through future cross-cultural research, fundamental questions regarding the concept of quality of life and how it is used in program services and supports to people with intellectual disabilities can be answered.

In building on their effort to validate the universality of certain properties of the eightdomain model of quality of life, Schalock et al. (2016) sought to create a theory of individual quality of life. By reading the available quality of life literature, they develop a conceptual model, integrate theory components, and apply and evaluate the theory. Results suggest that the theory it useful to the field of quality of life in that it can be used for problem solving and generalizability across diagnostic groups. The authors suggest further empirical research to test the theory to determine if application of the theory will reduce the anomalies associated with 
earlier approaches to people with intellectual disabilities and enhances their subjective and objective quality of life.

\section{Quality of Life for People with Visual Impairments}

Sheffield (2014) builds on the work of Schalock and Verdugo (2002) by adapting their validated instruments to suit the needs of the population she studies. Through her exploratory research, she defines themes specific to the quality of life for people who are blind. Some of these themes are similar to those outlined by the IASSID, however, many of them are more specialized due to the unique needs of a person with a sensory impairment. The emerging themes from Sheffield's (2014) study relevant to the phenomenon under investigation in my study are independence and knowledge and skills. While there are many other interrelated themes to emerge in Sheffield's (2014) work, these major themes contain subthemes that relate to a person who is blind's ability/inability to drive.

Self-determination, self-reliance, and self-advocacy are all subthemes that emerge under the major theme "independence" in Sheffield's (2014) study of quality of life for people who are blind. While recognizing all three of these subthemes are important, I mainly draw attention to self-determination as I continue to make connections between it, driver's education, quality of life, and independence. Self-determination concerns a person's ability to evaluate options and make choices. In Sheffield's (2014) study, participants note that self-determination requires having options to choose from along with the freedom to choose the option best for them. Instead of requiring or automatically waiving driver's education courses, I argue it would be beneficial to give students the opportunity to choose. While COMS believe the information discussed in driver's education courses is important, I set out to learn whether students with visual impairments feel taking part in driver's education classes is useful to their development and 
understanding of the built environment. The findings will help influence practice among TVIs and COMS, including giving students the opportunity to self-determine regarding driver's education courses.

The ability to drive has long been an outward sign of independence and a perceived step toward adulthood. If a student is unable to drive due to a disability, then it is important for students, parents, and professionals who work with the visually impaired to understand what other knowledge and skills are needed to compensate for this challenge to independence. The knowledge and skills related to quality of life presented in Sheffield's (2014) study include O\&M, braille, daily living skills, math, writing, and career and college preparation. All these subthemes are important to the overall quality of life for a person who is blind because they are the skills that have been shown to predict successful transition and employment outcomes (Wolffe \& Kelly, 2011; McDonnall, 2011, respectively). Positive experiences positively affect quality of life because they improve a person's confidence and self-efficacy. As I outline the context for my research study, I focus on O\&M as it most closely pertains to the phenomenon I intend to study because the ability to move around one's environment safely and efficiently is a critically important component of independence and independent travel. Independent travel, regardless of the method, is necessary to engage with the outside world. From getting groceries to running errands, the problem-solving required during independent travel also helps the social development of the traveler because he must interact with others, even if he is uncomfortable or shy. Although levels of independence vary across individuals, the empowerment that orientation and mobility can provide is noteworthy. It opens a world that was once closed off to those with vision loss and motivates them to explore their environment safely. Next, I synthesize the 
varying definitions of quality of life to create the operational definition of quality of life I use for my study.

\section{Operational Definition of Quality of Life}

Of the eight domains described by Schalock et al. (2005), emotional well-being and selfdetermination, and rights are the most pertinent to the phenomenon under investigation. Elements of emotional well-being and self-determination are contained in Sheffield's (2014) theme of independence. This is because all of these areas have a central focus on control. A major issue for nondrivers is a loss of control and predictability in their lives. This loss can be devastating. In addition to Sheffield's (2014) independence and knowledge and skills themes, I include the IASSID's rights domain to explore driver's education for nondrivers. People have the right to equal treatment, however, are we thinking of people with visual impairments equally in terms of driver's education? Should we be considering driver's education as part of their transitional program or are we denying them the opportunity to learn the information because they do not meet the visual requirements for licensure? In the following, I draw from the history of transition, independence indicators affecting transition, and the transition experiences of people with disabilities to illuminate the importance of considering these questions.

\section{Secondary Transition}

\section{Transition Definition and History}

Life is filled with transitions. We experience these transitions starting at a young age as we advance through grade levels and move from elementary school to secondary school and from secondary school to adulthood. For some, passing from secondary school to adulthood can be the most difficult of these transitions. Research has shown that since the 1980s, students with disabilities have consistently demonstrated poor post-secondary outcomes (Test, Mazzotti, \& 
Mustian, 2012). Various studies identify problems that initiated the transition movement of the 1980s. One study indicates students served in self-contained classrooms are not employed as often as those who participate in the general education curriculum (Hasazi, Gordon, \& Roe, 1985). A possible factor to explain this may be that students at the time in self-contained settings did not have access to vocational training with their nondisabled peers (Hasazi, Gordon, \& Roe, 1985).

McDonnell, Wilcox, and Boles (1986) expose the failure of students with disabilities to access appropriate vocational and residential services following school, adding to the high unemployment rate of people with disabilities, potentially negatively affecting their quality of life. Their work brings to light the need for more thorough transition planning by the education and rehabilitation systems. To lessen the gap in unemployment between those with disabilities and those without, leaders in the field began to focus on transition-focused education. The goal of transition-focused education is "to prepare students for post-school life" (Test, Mazzotti, \& Mustian, 2012, p. 337). In 1984, Madeleine Will, the Director of the Office of Special Education and Rehabilitation Services (OSERS) defined transition as:

An outcome-oriented process encompassing a broad array of services and experiences that lead to employment. Transition is a period that includes high school, the point of graduation, additional post-secondary education or adult services, and the initial years of employment... The transition for school to work and adult life requires sound preparation in the secondary school, adequate support at the point of school leaving, and secure opportunities and services, if needed, in adult situations. (p. 2)

"Transition" originally grew from concerted efforts to integrate students with disabilities in public schools after legislators passed the Education for All Handicapped Children Act of 
1975 (2016). Research in the area of transition outcomes was revolutionized when the Department of Education's Office of Special Education Programs (OSEP) sponsored the National Longitudinal Transition Study (NLTS) program (Blackorby \& Wagner, 1996). To more accurately examine post-school transition outcomes for a nationally representative sample of youth with disabilities, SRI International conducted two NLTS studies, one beginning in 1985 (Blackorby \& Wagner, 1996) and the other beginning in December 2000 (Wagner et al., 2003). The first NLTS includes data from "more than 8,000 youth ages 13 to 21 in special education in secondary school in 1985" (Blackorby \& Wagner, 1996, p. 401) and data were collected in two waves, one in 1987 and the second in 1990. The purpose of these studies was to illuminate the characteristics of youth with disabilities and their educational experiences, social activities, postschool employment, independence, and use of adult services (Blackorby \& Wagner, 1996). For the second NLTS, or NLTS2, data were collected for students aged 13 to 16 years receiving special education starting in December 2000. Participants were ages 21 to 25 years old during the final data collection wave in 2009 (Wagner et al., 2003).

Between the time when the final data collection wave of NLTS ended and the first wave of the NLTS2 began, transition services were strengthened with the Individuals with Disabilities Education Act of 1990 and its reauthorization in 1997 (Individuals with Disabilities Education Act [IDEA] of 1990, 2016; IDEA of 1997, 2016). IDEA and, more recently, the Individuals with Disabilities Education Improvement Act of 2004 (Individuals with Disabilities Education Improvement Act [IDEIA] of 2004, 2016), require the individualized education program (IEP) team to consider transition services by age 16. It is important to start early when creating a plan to ensure ample time to address all of a student's needs prior to the transition to life after high school. Some substantiated best transition practices include "paid or unpaid work experience, 
general education inclusion, daily living skills training, and self-determination skills training" (Landmark, Ju, \& Zhang, 2010, p. 165). Regardless of the degree of visual impairment or if there exist additional disabilities, it is crucial to strive to achieve some form of all best practices because they also act as opportunities for students to demonstrate their independence. In schools where the population is limited to students with disabilities, partaking in general education inclusion may not be an option; however, arranging opportunities for students to interact with peers without disabilities as often as possible should be a priority as this more closely reflects what they will experience in "the real world" (Landmark et al., 2010). This also allows students with visual impairments to utilize independent travel and self-determination skills in a variety of settings. Next I examine independence indicators known to play a role in successful post-school transition outcomes (Wagner et al., 2003).

\section{Independence}

Public perception of the independence of people with disabilities has evolved over the past century. Prior to the current "social model" of disability, there existed a "medical model" of disability that painted these individuals as victims or patients who needed to be helped or treated (Wolfensberger, 1983). This less-than-ideal perspective left people with disabilities little room for independence or the opportunity to make their own choices. Prompted by the deinstitutionalization movement, heightened public awareness, an increase in advocacy, and support from legislation, an environment for change was created (Wagner et al., 2003). The previously accepted medical model gave way to the social model of disability that is in practice today. The social model focuses on the individual rather than the impairment and emphasizes access, community, health promotion, and independence (Patrick, 1997). 
A major component of a successful transition from one stage of life to another is a wellthought-out and clearly defined plan for reaching an individual's goals. Students who are expected to take ownership and responsibility for planning their futures and engaging in selfdetermination in secondary school also transfer that responsibility into their lives after school (Malian \& Nevin, 2002; Price, Wolensky, \& Mulligan, 2002). The skills studied in the NLTS2 associated with the emerging independence of youth with disabilities transitioning from school to adulthood include but are not limited to: "(a) functional cognitive skills, (b) being mobile, (c) persisting in completing tasks, (d) self-advocating, (e) participating in transition planning and making progress toward independence-related transition goals, (f) and earning driving privileges" (Wagner et al., 2003, p. 6-2). While a number of additional emerging independence indicators are included in the study, the listed items are the most relevant to the phenomenon being explored. All these indicators are in some way related to self-determination, self-efficacy, and one's ability to travel independently. Next, I describe how youth with visual impairments fare in comparison to other disability groups in exhibiting the factors affecting a person's ability to achieve independence.

According to Wagner et al.'s (2003) report on the emerging factors of independence for youth with disabilities, youth with a visual impairment as their primary disability are among the highest scoring group in self-advocacy and persisting in completing tasks. Additionally, onefourth of these individuals are reported to have held a leadership role in planning their own transition and in making progress toward their transition goals (2003). By teaching students the skills to participate in planning their transition from student to adult, TVIs and COMS are providing them opportunities to practice self-determination. 
In terms of skills that support independence (self-care, functional cognitive skills, and mobility) and emerging independence in the community (earning driving privileges and having paid employment outside the home), however, students with visual impairments are among the least-skilled group. More specifically, about one-third of youth with a visual impairment performed in the low range when exhibiting mobility skills as reported by school staff (2003). This is a major issue because orientation and mobility are among the skills necessary to realize a positive quality of life and achieve independence (Sheffield, 2014). This reduction in independence negatively effects a person's transition experience and her overall quality of life.

Two additional factors influencing independence for youth with disabilities are having regular paid jobs and a driver's license or learners permit. Across disability classifications, " $41 \%$ of youth who have regular paid jobs also have a driver's license or learner's permit" (Wagner et al., 2003, p. 6-9). According to parent interviews, youth with visual impairments, deaf-blindness, orthopedic impairments, autism, and multiple impairments of the appropriate age for obtaining a driving permit or license are the least likely to drive. The degree of a person's visual impairment and the state restrictions for driver's license eligibility likely disqualify the majority of the VI population. This relationship between the ability to drive and job acquisition is troublesome for those who are not able to obtain a driver's license due to the nature of their disability because of the close linkage between driving ability and gaining a job.

While the NLTS2 provides a large sample size and exposes holes in transition experiences, it does not examine why these results exist, it simply indicates that they do. The awareness of the discrepancies between the highest achieving groups (youth with learning disabilities, speech impairments or other health impairments) and those with whose primary disability category is a visual impairment creates hope that this recognition of disparity in ability 
across disability category will spark action. Further analysis of the transition experiences of youth with visual impairments as indicated in the NLTS2 may help to inform professional practice. It is the responsibility of researchers to investigate possible factors and recommend solutions to close the gap in positive transition outcomes for individuals with disabilities so as to improve employment ratings and achieve greater independence.

Driver's education, in and of itself, does not appear relevant to the visually impaired. Upon further investigation, however, families and students with visual impairments often have to decide if the child should participate in driver's education classes based on school district's graduation requirements. In addition, vision requirements for each state are so strict that most adolescents who qualify for vision services in public schools (defined as "an impairment in vision that, even with correction, adversely effects a child's educational performance;" IDEA of 1990, 2016, Section 300.8.c.13) will have vision so low that it makes them ineligible to drive a motorized vehicle. For these reasons, examining the experiences of those who will never be able to drive is significant because, as research shows, life as a nondriver has lasting effects on the social inclusion, emotional well-being, personal development, and self-determination of people with visual impairments, regardless of age or life stage (Corn \& Rosenblum, 2002; Corn \& Sacks, 1994; Sacks \& Rosenblum, 2006).

The research presented in this section focuses on the experiences of youths with various forms of disability. In the following, I examine research specific to the transition experiences of those with visual impairment as their primary disability category and how instruction in the expanded core curriculum helps to meet the unique transition needs of this population. 


\section{Transition Experiences of Individuals with Visual Impairments}

One must have confidence in one's own efforts and abilities in order to transition successfully to the next life stage. This self-reliance is one aspect of the overarching theme “independence" uncovered in Sheffield's (2014) study. Independence and the desire to "do something meaningful" relate to transition in that both may act as motivators for people with visual impairments to seek employment and earn enough money to leave their family home.

The inability to procure a job over an extended period of time can crush a person's selfefficacy and discourage independence. Sadly, employment rates for people with visual impairments are abysmal. Only $43.7 \%$ of non-institutionalized people ages $21-64$ with a visual disability are employed according to the 2016 American Community Survey (Erickson, Lee, \& von Schrader, 2017). Researchers have attempted to determine predictors or aspects of transitional programs that contribute to successful employment outcomes for people with visual impairments. Shaw, Gold, and Wolffe (2007) conducted a quantitative study to investigate the effects of degree of vision loss, age, and sex on the employment status, job-search preparation, and job-search strategies of Canadian youths. Three hundred twenty eight 15-30-year-olds with visual impairments across Canada were surveyed. Results indicate young Canadians with visual impairments have low employment rates despite levels of education and that many youths spend little time actively searching for jobs or submitting job applications. The authors suggest more research is needed to explore the effectiveness of participants' job-search strategies. As well, actively coaching people with visual impairments in job-search strategies has the potential for great improvements to a person's quality of life. By broadening the number of applications submitted and increasing the likelihood of acquiring employment, the authors maintain job 
seekers with visual impairments are more likely to reduce financial dependency on others and improve their perceived self-efficacy by experiencing a successful outcome.

Finding a job is directly related to a person's perceived quality of life because earning an adequate salary to produce financial stability and autonomy while doing something meaningful can improve a person's perceived self-worth. To further the cause of determining predictors of successful transition, Wolffe and Kelly (2011) conducted a secondary analysis of the NLTS2 of transition-aged youth. The authors sought to determine whether ECC-like activities positively influence the likelihood of employment, participation in volunteer activities, or entering postsecondary school or training for youth with visual impairments. The relevant survey items in the NLTS2 were braille instruction, O\&M, assistive technology, and career counseling. Career education was not specified; however, students and parents were asked if youths received help in finding a job, training in job skills, or vocational education. The results indicate a significant relationship between youths who are blind or with low vision having a paid job and having received career counseling in three of the four waves of the NLTS2 (Wolffe \& Kelly, 2011). Additionally, in one of two waves of the NLTS2 under analysis, researchers identify a significant relationship between youths with low vision or blindness having been employed or involved in postsecondary training and having a paid job (2011). Also, there are strong relationships between youths who are totally blind who received braille and O\&M instruction and having a paid job outside the home (2011). The authors suggest these preliminary analyses demonstrate the importance of training in disability-specific skills for children who are visually impaired, calling for additional research to be conducted to address some of the other ECC areas that were not evidenced in their study, including self-determination. Wolffe and Kelly's (2011) study is significant not only because of its findings but also its sample size. Access to responses in the 
NLTS2 provided researchers a much larger sample of people with visual impairments, allowing researchers to generalize results to the greater population of people with visual disabilities in the U.S.

Instruction in key areas of the expanded core curriculum (e.g. social skills training and O\&M) continues to be invaluable to the independence of young adults with visual impairments. This is demonstrated in McDonnall's (2011) univariate analysis of the NLTS2 to examine 22 possible predictor variables and their effects on employment for employed transition-aged youth with visual impairments. Participants available using the NLTS2 include a representative sample of people with visual impairments across the U.S. Results indicate early and recent work experiences, completion of a postsecondary program, difficulty with transportation, independent travel skills, and social skills were predictors of employment. The researchers suggest more attention be spent on these six skill areas to assist in smoother transition of youth with visual impairments into the world of work, all of which foster greater independence, thus, improving quality of life.

As I argue previously, degree of vision loss does not necessarily predict successful employment or transition outcomes. Instead, research concludes focus should be placed on early work experiences, independent travel skills in the form of O\&M instruction, braille (when necessary), postsecondary training, and social skills. A deficiency in any of these indicators can make it more challenging to find employment, thus affecting a person's independence and quality of life and ability successfully to transition to independent living.

One of the ways educators can devote attention to at least four of the indicators linked to positive transition outcomes is by teaching the expanded core curriculum. The expanded core curriculum is the means by which people with visual impairments receive a transition-focused 
education. The nine areas are typically addressed in some form or another in residential schools, public schools, and rehabilitation centers to minimize the potentially disabling effects of vision loss.

\section{Expanded Core Curriculum (as a vehicle for secondary transition for those with VI)}

Instruction in the areas of the expanded core curriculum is necessary to ensure the success of youth with visual impairments as they enter adulthood. The commonly accepted, nine skill areas of use of assistive technology, career education, compensatory skills, independent living, orientation and mobility (O\&M), recreation and leisure, self-determination, sensory efficiency skills, and social interaction skills are recognized by the Council for Exceptional Children (CEC) as essential aspects of educating youth with visual impairments. The CEC includes all nine areas of the ECC in its list of required "knowledge and skills beginning TVIs" (Sapp \& Hatlen, 2010, p. 342) must have upon graduation (Council for Exceptional Children, 2009). Although there are nine widely accepted areas of instruction that young people with visual impairments require in order to prepare them to enter post-secondary life, two are most closely linked to the topic of nondriving and its effect on quality of life. Instruction in orientation and mobility and self-determination are the means by which youth with visual impairments demonstrate their independence and are linked to positive transition outcomes (McDonnall, 2011).

Orientation and mobility. Orientation and mobility is a relatively "young" field, yet it is of monumental importance to an individual's independence. Imagine losing your vision. You would likely feel apprehension and fear about traveling, or think independent travel is now impossible. This can result in a feeling of entrapment within one's home environment. Orientation and mobility training is the way people with a congenital (at birth) or adventitious 
(acquired after previously having vision) visual impairment regain the power to take back their independence. This is because O\&M is the systematic way in which people with visual impairments are taught to "orient themselves to their environments and move as safely, efficiently, and independently as possible in those environments" (Sapp \& Hatlen, 2010, p. 340). Certified orientation and mobility specialists begin by helping children understand their bodies and basic movement in space. The goals of COMS are to teach students the skills to navigate complex environments safely, cross streets, and access transportation so they can travel independently. Eventually, they progress to learning how to plan routes in a variety of environments. In the following, I briefly outline the development of O\&M as a discipline in the U.S. to demonstrate its value as an integral component in the educational program for people with visual impairments.

The development of formal training centers for dog guide use exposed the need for formalized training in orientation and mobility. In the 1920s (almost 100 years after the first U.S. residential blind school was established), people with visual impairments were trained to use dog guides to travel independently (Hatlen, 2000). A commonly held belief is that the dog tells the dog guide handler where to go. This is not true. The handler gives the dog commands; the dog is responsible for keeping him safe when approaching obstacles. The handler must have strong orientation and mobility skills in order to have a successful handler-dog guide team. If the dog guide user does not know where she is going, the dog is not going to get her to the destination. This is why strong orientation and mobility skills are vastly important.

Now fast-forward to World Wars I and II. The increase in formal orientation and mobility programs was a byproduct of veterans returning from these wars. The large number of blinded veterans prompted Richard Hoover at Valley Forge General Hospital and Russell Williams at 
Hines Hospital (rehabilitation centers for veterans during WWII) to employ the long, white cane as a mobility device (Miyagawa, 1999). During this time, the "Hoover cane" was invented and systematic instruction was provided based on the cane as a tool for travel, not a sign of blindness. In residential schools for the blind, "foot travel" was taught by TVIs and by the 1950s, many teachers decided they wanted to teach approaches to independent travel to students in schools (Hatlen, 2000). Previously, consultants from the American Foundation for the Blind (AFB) had held summer workshops at universities for TVIs so they could teach basic travel techniques but did not provide comprehensive O\&M training (Hatlen, 2000, p. 25). This may have included human guide technique (walking with a person who is sighted by holding onto her arm), trailing (following the wall with one hand), and using protective techniques (one arm in front of face and other in front of hips) when traveling without a cane. Only certified orientation and mobility specialists were qualified to teach cane training. When COMS decided they wanted to work in schools, cane training became part of the curriculum for students in both residential schools and in public, day schools (Hatlen, 2000).

As is often the case, demand created a need for formal training programs for orientation and mobility specialists. In 1960, Boston College established the first university-based O\&Mspecialist training program (Hatlen, 2000). Many universities followed suit to meet the growing travel needs of individuals with LV/B. Initially, O\&M was taught only to adults but by the mid1970s, almost half of all COMS taught children in schools (Hatlen, 2000). Orientation and mobility's importance was realized during the 1970s when it became a required course for students with visual impairments. It was also the first disability-specific educational need (in addition to braille) deemed necessary for students with LV/B (Hatlen, 2000). 
A large part of O\&M training involves taking in information and making safe decisions based on that information. This is one of the hallmarks of self-determination. In the following section, I describe the importance of self-determination to positive post-school outcomes for youth with visual impairments.

Self-determination. In order for a person with a visual impairment to be able to travel independently or obtain a job, he must believe that he can do it. Bandura (1977) introduced the concept that a person's ability to complete a task is directly linked to the strength of his belief he can accomplish the task. This determines whether a person will attempt a task, how hard he will try, and how long he will persist through obstacles. Self-efficacy has had a significant effect for rehabilitation personnel struggling with unmotivated or under-motivated clients because it influences how a person is able to perceive and respond to her environment. This is because a person will not attempt a task if he does not believe he can complete it. A person's perceived self-efficacy comes from one or more of the following sources: (a) performance accomplishments, (b) vicarious experiences, (c) verbal persuasion, and (d) monitoring of one's own psychological states (Welsh, 1997). The most significant of the four sources of perceived self-efficacy are performance accomplishments. When a person has direct experience successfully performing a task, it raises his efficacy judgment. He then believes he can do most tasks similarly to those that he has completed successfully. This is why COMS sequence lessons in such a way that clients will experience small successes to build their self-efficacy. Repeated failures lower self-efficacy, especially if they occur early in instruction.

It is the responsibility of the adults in a child with a visual impairment's life to help the child develop self-determination skills. Self-determination is a person's right freely to choose (without excessive influence), how she wishes to live her life (Hatlen, 2000). She must be taught 
the choices that are available to her, learn the skills necessary to make the most of these choices, and be given the opportunity to choose what is best for her. A large part of this process is instructing the person with a visual impairment to evaluate options and make choices.

Although Erikson's (1950) model of psychosocial developmental needs is limited in describing the complexities of identity construction, it can (and should) be applied to instruction in self-determination for people with visual impairments. Erikson theorized people progress through eight psychosocial states that build upon one another, starting at birth and ending in late adulthood (See Table 1). The extent to which each subsequent need can be met is directly linked to the extent to which each prior need has been met. For example, if a child is ashamed of his visual impairment or feels doubt about his abilities (age 1-3 years), then it will make it much more difficult for him to develop the initiative (age 4-6 years) to complete tasks. This is why it is even more important that children who are blind or have low vision encounter positive interactions with their parents and others in their environment. They need to be supported as they explore and are introduced to new experiences if they are to become self-determined and develop independence.

\section{Table 1}

Erikson's Stages of Psychosocial Development

\begin{tabular}{llll}
\hline \multicolumn{1}{c}{ Stage } & \multicolumn{1}{c}{ Ages } & \multicolumn{1}{c}{ Stage } & \multicolumn{1}{c}{ Ages } \\
\hline $\begin{array}{l}\text { Basic trust vs. basic } \\
\text { mistrust }\end{array}$ & $0-1$ years & $\begin{array}{l}\text { Identity vs. role } \\
\text { confusion }\end{array}$ & $12-18$ years \\
$\begin{array}{l}\text { Autonomy vs. shame } \\
\text { and doubt }\end{array}$ & $1-3$ years & Intimacy vs. isolation & $\begin{array}{l}\text { Young } \\
\text { adulthood }\end{array}$ \\
$\begin{array}{l}\text { Initiative vs. guilt } \\
\text { Industry vs. }\end{array}$ & $7-11$ years & $\begin{array}{l}\text { Generativity vs. } \\
\text { stagnation }\end{array}$ & $\begin{array}{l}\text { Middle } \\
\text { adulthood }\end{array}$ \\
$\begin{array}{l}\text { inferiority } \\
\text { Anted }\end{array}$ & Ego integrity vs. despair & Later adulthood \\
\hline
\end{tabular}

Adapted from Hatlen, 2000, p. 163 
Despite the importance and attention placed on self-determination, research shows, when compared to people without disabilities, youth and adults with disabilities report lower levels of self-determination. Lipkowitz and Mithaug (2003) test the disability-effect (disability affects adjustment and self-determination) and group-identity (students with strong group identities have higher self-determination ratings than students with weak group identities) hypotheses by comparing adjustment and self-determination ratings of students with sensory disabilities. Participants were 209 students representing a range of age, grade level, and ethnic background. Findings indicate support for the disability-effect hypothesis in that students in more restrictive special-education placements tend to rate lower their adjustment capabilities than do students in more inclusive settings. Educational placement and student ratings on self-determined adjustments are correlated for both students with visual impairments and students who are deaf or hard-of-hearing. Interestingly, students with LV/B rate their self-determined adjustment capabilities significantly higher than students who are deaf or hard-of-hearing.

Correlation between educational placement and student ratings is not surprising because students in more-restrictive environments receive more support than those in less-restrictive environments. Such increased support may cause students to believe they require assistance with decision-making or when completing difficult tasks independently, affecting their perceived ability to self-determine. Self-determination is an important part of decision-making, which is also pivotal during independent travel. It is crucial students with visual impairments in all special education placements are provided instruction in self-determination and plenty of opportunity to demonstrate self-determined decision-making in preparation for adulthood. Self-determination has been identified as a valid predictor of positive learning outcomes (Hatlen, 2003), and thus is a major component of transition experiences. Students who are self- 
determined are able to make decisions about their futures. This includes knowing what they want and creating a plan for achieving their goals in a variety of circumstances. One important area is traveling independently.

\section{Independence of Travel}

\section{Factors Affecting Independence}

Sighted peoples' attitudes toward blindness and people who are blind play an integral role in the development of self-concept and perceptions of self-efficacy of people who are blind; this is because attitudes affect the feedback a person who is blind receives (Welsh, 1997). Attitudes among the sighted influence the person who is blind's judgments of his own abilities and also affect the amount of help he is offered as he travels. If a person is over-helped, she can come to rely too heavily on assistance of others even when she has been taught the skills to problem-solve. In addition, an over-projection of fear onto a person with a visual impairment can significantly impair his ability to set high expectations for himself and attain his goals, especially in the arena of independent travel. Four areas that can inhibit and/or promote independent travel by people with visual impairments are disability characteristics, learned helplessness, culture, and family.

Disability characteristics. People's beliefs about a particular group are often rooted in their past experiences of said group and can influence whether they accept a group has the capacity for independence. This is true of racial groups, socioeconomic groups, cultural groups, and even disability groups. The belief a person has about a group can also have an effect on her attitude toward and expectations of the group. If a person with vision has had a negative experience with a person who is blind, the person who is blind may be seen in a way that is not reflective of her actual abilities. When a person has a positive experience, he may tend to over- 
sensationalize it and regard the person as exceptionally talented, even though the person with a visual impairment is performing a routine task (Welsh, 1997). For example, when a person who is blind travels up stairs alone using his cane or boards a bus by himself, oftentimes, sighted observers comment on the cane user's "bravery" or "superior skills" when he is completing a routine task he does each day.

Tringo (1970) theorizes there exists a hierarchy of preference for disability categories. Where a person places a specific disability on this hierarchy is influenced by his feelings toward or experiences with a disability group. The top three categories are physical disabilities, sensory disabilities (blindness and deafness), and brain disabilities (Tringo, 1970). Although ranked relatively highly on the overall hierarchy, certain attitudes about visual impairments lower others' expectations and can make it difficult to obtain and maintain employment.

One major barrier to reasonable expectations of people with visual impairments is the attitude of the employer (Clayton, 1983). In Clayton's (1983) study, employers thought jobs were too technical or dangerous for workers who are blind or visually impaired. In addition, employers are fearful their workers' compensation premiums would increase with the employment of a person with a visual impairment. This attitude persists despite the fact that workers with visual impairments are more motivated, can be more productive, tend to be more loyal, demonstrate lower levels of absenteeism, and have an impressive safety record (Clayton, 1983).

Another characteristic that plays a role in the public's attitudes toward visual impairments is the lack of eye contact on the part of the person who is blind or has low vision. In addition, people with low vision or blindness may exhibit atypical gestures or facial expressions or are unable to read visual conversational cues used by the sighted person (Monbeck, 1973). The 
sighted person may think the person with a visual impairment is uninterested or incapable of understanding. Characteristics of a person's disability are not the only influence on societal expectations of people with visual impairments; learned helpless behavior guides them as well.

Learned helplessness. Learned helplessness is a debilitating learning outcome which stems from the belief that a person's failure to complete a task is due to a personal lack of ability rather than lack of effort. Unfortunately, learned helplessness is all-too-common among students with visual impairments. For typically achieving students, Dweck (1975) and Dweck and Licht (1980) identify two types of responses to failure. First, "mastery-oriented" students respond to failure by increasing their efforts and attribute success to personal ability and failure to a lack of effort. These students also demonstrate greater powers of concentration and persistence in the face of failure. The second type of typically achieving students respond to failure with "learned helplessness." To these students, failure appears to be devastating and results in decreased effort and lowered persistence. "Learned helpless" students expect to fail, use poor problem-solving strategies, and attribute failure to a personal lack of ability. Instead of attributing success to internal factors, they attribute them to luck or a result of task ease. As a result, students who exhibit learned helpless behaviors have more difficulty with independent travel to new environments because problem-solving is required. Experiences of failure further decrease the person's self-efficacy and lead to the self-fulfilling prophecy that the person is unable to travel independently without assistance. This slippery slope is why providing instruction that allows the person with a visual impairment small successes in the early stages of travel is so important.

Learned helplessness is a major concern of professionals who work with people with disabilities. In students with visual impairments, it can be a result of the dependency caused by a visual impairment and the reactions of others to the disability. Early in life, but continuing into 
young adulthood, children with a visual impairment rely heavily on family members to describe and interpret visual information in their environment. As a result, the child's feelings of selfconcept and self-worth become strongly linked to her parent's attitude and acceptance of her disability (Barraga, 1983).

Oftentimes peers, teachers, and parents are quick to assist the "poor blind child" before he has the opportunity to make a mistake. When this occurs, it robs the child of the opportunity to experience and learn from his failures and successes. Instead, others' actions tell the child she is unable to complete a task without the assistance of others. This has a negative effect on learning and independence and results in long-term displays of passivity by the student with a visual impairment. It also lowers his and others' expectations for his performance.

Little empirical research had been conducted to determine specific characteristics of learned helplessness and low self-esteem for students with visual impairments, making it difficult to create an action plan to combat these issues. In an effort to add to the body of literature, Head, Pysh, Chalfant, and Spencer (1992) conducted a pilot study to identify cognitive-motivational aspects of achievement for 14 students with visual impairments in grades three through six in Arizona. Some students were served at the state school for the blind, while others received their education in public schools. The purposes for the one-year pilot study were three-fold: (a) adapt or develop practical assessment instruments that could be used by teachers to measure learned helplessness in students with visual impairments, (b) use the assessment instruments to describe learned helplessness among students who are blind or visually impaired in the state school for the blind and in the public schools, and (c) develop a conceptual intervention model for classroom use by TVIs to combat learned helplessness (Head et al., 1992). Results helped researchers develop assessment techniques used to describe the 
characteristics of learned helplessness among students with visual impairments. Many participants fell into the learned helpless range based on their responses to the assessment instrument. The "mastery oriented" students were only within one point of the "learned helpless" range. This could mean that although some participants were labeled "mastery oriented," they still exhibited some "learned helpless" behaviors. The implications of these findings are paramount. The authors suggest teachers and family members should help make children with visual impairments aware of the reasons why they succeed or fail in all areas of life. They should be taught causational thinking and given language to express it. The authors also suggest that parents and teachers carefully examine the kind and quality of the feedback they provide to students with visual impairments about successes and failures. This knowledge will help to increase others' expectations of the person with a visual impairment by reducing learned helplessness (Head et al., 1992). In addition, an increase in expectations may assist in improving the student's self-efficacy and foster increased independence in an effort to meet those higher standards. In contrast to the detriment learned helplessness causes to a person's independence, culture may either inhibit or promote independence of travel for a person with a visual impairment.

Culture. All people are shaped by culture, including those with visual impairments. Cultural influences may include race, cultural background, gender, sex, age, and socioeconomic status. When discussing culture, it is difficult simply to peel away layers to determine which of the previously listed variables carries the biggest impact on others' perceptions. Unless examined within the context of a specific aspect of culture, an individual will likely arrive at perceptions more stereotypical than evidence-based. While there are commonalities within and across aspects 
of culture, an individual's experience and perception is unique based on the elements of race, cultural background, gender, sex, age, and socioeconomic status, among other aspects.

Cultural factors play an integral role in a family's values and beliefs. They also affect the way family members respond to and interact with a child with a visual impairment. These values and beliefs may be influenced by a number of factors including family values, length of residence in the dominant culture, educational level, socioeconomic status, and beliefs about people with disabilities (Chung, 1996; Hyun \& Fowler, 1995; Pang, 1995). For example, a parent uproots her family from an island in the South Pacific and moves to the U.S. so her son who is blind can be educated. In his birth country, he likely would not have realized the independence he has today; instead, he would be kept at home because schools do not have access to professionals to teach O\&M, self-determination, or assist with transition (E. Ngirmekur, personal communication, October 17, 2012). Cultural factors also affect the way families interact with professionals. It may seem as though families do not care about a child when they dismiss the recommendations of a teacher, however, the suggestion may be at odds with the families' culturally based values.

Some cultural protocols can create barriers for people with visual impairments. In BevanBrown and Walker's (2013) qualitative study of 10 Māori adults with visual impairments, they find that some aspects of Māori culture made it difficult to access community areas or engage in cultural activities and experiences. One participant was displeased that although he was a fluent Māori speaker, he was rarely asked to speak in the traditional meeting area. He believes his visual impairment makes others underestimate him. Results indicate the majority of barriers participants experience are related to acceptance and accommodation of visual impairment in general, with few barriers specific to Māori culture. The authors instruct educational 
professionals to take into consideration an individual's culture when planning the education and services of people with visual impairments.

Culture is not simply limited to racial or ethnic background. It also includes other elements. Research regarding race, socioeconomic status, gender and age as they relate to others' expectations of people with visual impairments is scant. Additional studies of different cultures could be used to provide further support but the message is the same: culture affects people's expectations of individuals who are blind or visually impaired. In addition, while family can be considered a part of culture, "family" is addressed in its own section apart from "culture" because the research available is specific to families' attitudes and expectations of family members with visual impairments. Next, I explain more in-depth about families' expectations and how these shape a person's movement toward independence.

Family. It may be surprising to some to find that families of people with visual impairments also hold many of the same attitudes about people with visual impairments as the general public. Negative and rejecting attitudes can have a debilitating effect on the person with a visual impairment (Large, 1982). As mentioned previously, a family's behavior has significant ramifications on the success of a child with a visual impairment. If a family projects its fears and doubts on the child, then he will likely believe he cannot successfully complete tasks that would make him independent. This is most evident with independent travel. Oftentimes, families do not trust that their child with a visual impairment will make a safe decision when crossing the street, for example. Instead, they require the child with a visual impairment to travel with a sighted sibling or adult. What does this tell the child? A healthy level of caution maintains safety, but an overwhelming feeling of fear will completely undermine any instruction by the COMS. If the 
family is supportive and confident in the student's ability to handle independent travel, then it will be a strong, positive force (Welsh, 1997).

Family opinion also shapes the way the person with LV/B views himself. Moore (1984) notes the importance of family in interpersonal development in that it affects how the person with a visual impairment perceives his visual impairment and how he chooses to act on that information. Families who are embarrassed by their child walking in the community with a cane will extend this feeling of embarrassment to their child. Children are less likely to use devices that will make completing tasks more efficient and safe if their families disapprove of these devices" use, making them even more intent on "passing" as sighted in a sight-dominated world.

Another circumstance which may challenge an individual with a visual impairment's independence is when families desire to restrict his independence because they fear not being relied upon. Some researchers investigate family dynamics that seem to be held together by the need for family members to care for one sick or dependent member of the family (Fordyce, 1971; Klausner, 1969). In their respective research, they find some families feel threatened when the person who is considered "dependent" gains independence and the family members are no longer able to fulfill their care-giving roles. This can result in behaviors that sabotage the education or rehabilitation process. Researchers also show that, in some instances, mobility training has activated a family crisis because instruction has made the family member with a visual impairment more independent (Fordyce, 1971; Klausner, 1969). One way to reduce the potential for such a family crisis is to communicate with family members about their hopes and expectations for the person with a visual impairment. Since family members' attitudes toward the person with a visual impairment have the largest influence on his behavior, it is important to educate them about the consequences of their expectations. 
In kind to family attitudes toward independence, families typically have strong feelings about their child's transition plan and future goals. In some states, school districts require students to take driver's education as part of their course of study. Unfortunately, driver's education is often overlooked and excluded as part of the "inclusive education" of a person with a disability (McGill \& Vogtle, 2001). As described in the IDEA, individuals with disabilities are people first. It is important to consider the potential value of driver's education to a person with a disability, even if she may not be able to earn a driver's license.

\section{Driver's Education}

\section{Drivers and Nondrivers with Disabilities}

People with disabilities often experience barriers that hinder their mobility, causing them to rely on others for transportation. Instead, they are forced to utilize resources that are overwhelmed and underfunded (e.g. paratransit; McGill \& Vogtle, 2001) or pay large sums of money to use a taxi if their destination is not within a reasonable walking distance of a fixed bus stop. This can be even more challenging for people with visual impairments who require an intersection controlled by a stop sign or stoplight to negotiate a safe crossing. As a result, people with disabilities experience significant limitations in regard to "competitive employment opportunities, engagement in leisure activities, and attempts to become self-sufficient" (McGill \& Vogtle, 2001, p. 455). McGill and Vogtle (2001), posit that people with disabilities should "have a driver's license whenever possible" (p. 456).

Unfortunately, driver's education teachers in public schools are not well equipped to provide instruction to meet the unique needs of students with disabilities. In the case of people with physical disabilities in Alabama, cars were not fitted with adaptive equipment to allow access and driver's education teachers were not trained to teach students how to operate the 
modified vehicle (McGill \& Vogtle, 2001). Additionally, the cost of private lessons can be expensive and arduous for the person with a disability learning to drive. Students without disabilities have access to driver's education at a nominal fee through their public school, why should this not also be accessible to those with disabilities?

Brooks, Mossey, Tyler, and Collins (2013) also conducted an exploratory investigation to determine if driving simulators were appropriate to teach pre-driving skills to four young adults ages 21 to 23 with intellectual disabilities. Their study was designed in hopes of stepping closer to establishing a driver's education program that would be appropriate for people with intellectual disabilities. Their findings indicate that the simulator was "an effective tool to provide a safe learning opportunity for young adults with intellectual disabilities to learn predriving skills" (Brooks et al., 2013, p. 211). The authors call for additional research that includes a larger sample size and to "explore specific skills and knowledge types that are predictive of driving abilities" (p. 212). They also hope that training in pre-driving skills may act as an intervention method and opportunity to determine candidates for independent driving (2013).

Driving is important to people with and without disabilities. The inability to drive is perceived drastically to reduce a person's job prospects and feelings of independence (McGill \& Vogtle, 2001), which has the potential negatively to affect quality of life for the visually impaired (and blind).

\section{Effect of Nondriving on Quality of Life}

One of the major milestones in a teenager's life is the ability to drive. It gives an adolescent higher standing among her peer group because she has greater independence and mobility (Sebald, 1983). A common theme in the research for driving and people with visual impairments is a lack of spontaneity. Corn and Sacks (1994) examine the effect of nondriving on 
adults with visual impairments and find that not being able to drive moderately affected their participation in social activities, chances to date, and obtain employment. Participants also expressed concern due to a lack of spontaneity because they "needed to plan activities that required independent travel" (Sacks \& Rosenblum, 2006, p. 212). Although researchers do not explicitly say it, their findings suggest that nondriving has an effect on interpersonal relationships and social inclusion, two significant measures of quality of life (Schalock \& Verdugo, 2002). Feeling as though one is unable to engage socially can be frustrating, especially for those who once lived a vibrant and interactional lifestyle. This may exacerbate the effects of vision loss by making the person feel as though in addition to her vision, she has lost other aspects of her previous life.

The effects of vision loss may be even more pronounced in older adults because it forces them to alter patterns and habits they have acquired over time. Similar to working-age adults, individuals age 60 and older with vision loss also indicate that ceasing to drive results in major lifestyle changes (Corn \& Rosenblum, 2002; Rosenblum \& Corn, 2002a; Rosenblum \& Corn, 2002b) and leaves individuals feeling reduced self-control and independence (Eisenhelder, 1990), because the driver's license can serve as a "symbol of self-worth" (Meier, 1992, p. 17). Older adults do not tend to be as active in work or volunteering as when they were able to drive, they change their living situation to access transportation, and do not participate as readily in leisure activities due to the additional planning and time required (Rosenblum \& Corn, 2002a).

Like adults with low vision, adolescents with visual impairments do not have the same opportunities spontaneously to visit friends, travel independently, or participate in ageappropriate activities (Sacks \& Rosenblum, 2006). Studies also show students with low vision spend more time alone and engage more in passive activities and in sleep than their functionally 
blind or sighted peers (Kef, 1997; Sacks, Wolffe, \& Tierney, 1998; Wolffe \& Sacks, 1997). In Sacks and Rosenblum's (2006) study of adolescents' perceptions of driving and nondriving, they find many participants are unclear about the nature of their visual impairment, have minimal information about driving with low vision, and are not familiar with strategies to deal with nondriving alternatives. In addition, they experience "high levels of frustration and feelings of great sadness" regarding their inability to drive. Due to the emotional implications found in Sacks and Rosenblum's (2006) study and the role of emotional well-being in quality of life, it is even more important to know if students with visual impairments who could potentially encounter negative feelings by attending a driver's education course perceive the information as valuable.

The issue of driver's education for people with visual impairments is complex. The potential psychological effects of participating in an activity that reminds a person that she is unable to achieve a certain milestone can be devastating. The information covered in driver's education, however, is important for the independent traveler who is visually impaired as both a pedestrian and passenger in a motorized vehicle. How does one reconcile these opposing viewpoints?

Through the review of the available literature I have examined predictors to successful transition for youth with visual impairments, including the role of independence and the ways in which driving (nondriving) connect with these phenomena. I also uncover factors influencing quality of life, one of which is independence. The gap in the literature I seek to fill with my study is the relationship of driving and driver's education to independence and the ways in which this is manifested in self-efficacy, identity construction, and transition outcomes for youth and young adults with visual impairments that have the potential to influence a person's quality of life. 


\section{CHAPTER III: METHODOLOGY}

\section{Theoretical Framework}

A researcher's theoretical perspective is important to disclose because it provides not only a context for the methodology of a study, but a key to the researcher's epistemology and ontology and therefore an understanding of the scope and reach of a researcher's truth and knowledge claims. Interpretivism is one theoretical perspective common in the social sciences, and the theoretical perspective to which I ascribe. The approach of an interpretivist researcher is to "look for culturally derived and historically situated interpretations of the social life-world" (Crotty, 1998, p. 67). Interpretivists seek to understand a phenomenon, not focus on causality in an effort to explain it. This theoretical perspective is based on a constructivist epistemology. In constructivism, the researcher believes there is no objective truth waiting for humans to discover; humans construct all meaning (Crotty, 1998). Interpretivists make no claim to generalizability in their truth and knowledge claims, that is, Interpretivists' research offers localized findings that represent one truth among many about those they study and which may be true of other like people or sites. The way such research can become applicable to a larger, social context is through the use of social theory as a framework through which to analyze data. Finally, Interpretivists make no claim to objectivity; they understand objectivity to be neither possible nor desirable and realize all data gathered by a researcher naturally flows through that researcher and his or her experiences, beliefs, subjectivities, and biases on the way to meaning making and truth and knowledge claims.

In this research study, I set out to define a very specific phenomenon: the effects of driver's education courses on the knowledge of and travel in the built environment of people with visual impairments. As demonstrated previously, there is no research that directly addresses 
this issue. This study signifies the first stage of exploratory work, defining the phenomenon, and thus, an important first step in a much longer line of inquiry, because definition of the phenomenon is required before researchers can look further at this topic.

\section{Biases and Subjectivities}

Naturally, my upbringing, experiences, and beliefs affect the way I interpret the data I collect. As an interpretivist, I make no claim to objectivity, so it is important I describe my biases and subjectivities as I understand them to disclose to readers that my experiences, for instance as a white, middle-class woman, raised in a Midwestern suburb, will influence the meaning I make from the data.

I grew up in a Christian home and have always had a heart for helping people. My most memorable experience with a person with a sensory disability was as a cashier at a grocery store while I was in high school. As I rung up a woman in the checkout line, I noticed her daughter becoming exceedingly more aggravated that her mother was not paying attention to her. The girl continued to sign to her mother to no avail. The cashier at the next register also observed this and started signing to the little girl. She was elated that someone could communicate with her. Seeing this, I wanted to be the source of joy for a child with a disability. This experience inspired me to work with the deaf. This, however, was not where my journey into special education ended. Upon arrival to my undergraduate university, I was told that there were no openings in the Deaf and Hard of Hearing $(\mathrm{D} / \mathrm{HH})$ sequence and that I would be placed on a waiting list. Unsure what my future held, it was recommended I follow the Low Vision/Blindness (LV/B) sequence in the Special Education program until there was an opening in the $\mathrm{D} / \mathrm{HH}$ sequence. In the spring semester of my freshman year, I received a phone call that there was an opening in the $\mathrm{D} / \mathrm{HH}$ sequence and asked if I would like to take part in the American Sign Language course. Later that 
day, I experienced a heavy conviction and resolve that $\mathrm{D} / \mathrm{HH}$ was not the path I was meant to take, LV/B was. This decision put me on the trajectory I am now. I have dedicated my professional life to helping people with visual impairments learn the skills required to be independent.

Growing up with a cousin with a physical disability, I learned at a young age that the disability does not define the individual; it was just one of her many unique qualities. Through perseverance, hard work, and a supportive network of adults and friends, she was able to attain the goals she set for herself. As I work in the field of visual impairments, I have observed a number of capable adults who are blind excel in their respective fields. Similar to my cousin, they need assistance on occasion, however, they are not helpless or passive spectators in their own lives. With every request that is made, it is the requestor who is still in control of the amount of assistance a person provides. The success of these self-determined, mastery-oriented individuals motivates me to strive to have my students achieve as much independence as is appropriate, given their unique situations.

In my early years of teaching students with visual impairments, I encountered a number of students in high school who struggled with a challenging question: "Do I have to take driver's education if I won't ever get to drive?” This is also a challenging question for administrators. Do they require students to participate in the class as a means to satisfy graduation credit? Or do they waive the student from the class because he will not achieve the same outcome of earning a driver's license as his peers? Taking into account a student's emotional concerns, but also considering the potential benefit of learning what drivers learn, this topic has confounded me for years. 
The goal of teachers working with the visually impaired is to "work yourself out of a job.” As students become more comfortable and adept at the skills addressed in the ECC, professionals tend to reduce the amount of time spent with the individual because his need for direct instruction in these areas has decreased. TVIs and COMS hope that by the time a student graduates, he will have all the skills necessary to enter adulthood. That being said, even the best teachers cannot control all factors related to student success. I have observed first-hand the effects of student motivation, family support, culture, and social class on students' transition to life after high school. If families are not aware of the options available to their children, then they are less likely to support those decisions. My experience has also been that, more often than not, families of a higher social class tend to push their children to pursue some form of education or training after high school. On the other hand, one working-class family with whom I worked was content with the individual with a visual impairment entering the workforce in a low-skill, low-paying job so he could contribute to the household. If parents and students have a certain plan for what life will be like post-secondary school, it is the teacher's responsibility to honor that vision and not impose her view of "success" on the family. I believe that as a TVI/COMS, it is my duty to empower the individual within the context of his family culture. That will look different from person to person based on severity of disability(ies), available resources, family culture, and family dynamic.

I have worked in rural, suburban, and urban settings as a TVI and COMS for 12 years. In this time, I have observed how family and student expectations can hinder or promote a student's independence. This is most evident in independent travel. In my experience, students rise or fall to meet the expectations of their families. This is especially frustrating as a COMS when what I am attempting to teach is underscored by projected fear and misgivings about a student's 
independent travel abilities. I feel strongly that education is the key to the larger world. It may be in a structured setting or it may be in an unstructured, "college of life" scenario. In either case, education opens opportunities that were once closed to the individual. As a COMS, I have certain beliefs about the importance of the material discussed in driver's education courses. I also believe that some students are well suited to participate and benefit from instruction from a trained professional in driver's education. I recognize my subjectivities and biases will affect my interpretation of the data.

\section{Population}

The population I study includes young adults aged 18-30 with low vision living in a midwestern state. Participants have either taken driver's education or read the Rules of the Road handbook and have a visual impairment that disqualifies them from obtaining a driver's license. Pseudonyms for people and locations have been used to ensure confidentiality of participants. The goal of this study is to examine their perspectives of the effect of driver's education on their ability to travel independently in a world dominated by motorized vehicles (busses, trains, automobiles). The reason I have selected young adults who are slightly removed from taking the course is because they will likely have more of a long-view perspective of the course's effect on their behavior, perceptions and attitudes, knowledge and practice. School-age students who are taking part in the course at the time of this study or are taking it shortly before the time of this study are not likely to have enough experiences or experiences that may be independent living, for example, that would allow them to determine later effects of and lasting knowledge from taking driver's education on their independent travel.

The broad range of ages to include adults $18-30$ was chosen so I would be better able to collect information from a substantive sample. Low vision/blindness is a low-incidence disability 
area. Given my own professional practice, I know locating an even-more-specific sample of this group will be challenging. If I limited the criteria to include a smaller age range or eliminated subjects with low vision or who are blind, I would likely not have had enough participants to conduct the study which might yield enough data to discover themes across participants. Due to the low-incidence of this criteria occurring among the general population, I utilized a convenience sampling strategy (Patton, 2002) because of the low incidence of potential participants within reasonable traveling distance from my location.

People with visual impairments who attend colleges and universities are those participants most likely to have the level of independent travel skills I seek. I solicited participants by contacting the office that provides accommodations for people with disabilities at two community colleges and two universities. The universities were good sources for subjects because both have personnel preparation programs in visual disabilities and it is well-known in the blindness community that it is common for people with a visual impairment to enter the field of low vision/blindness. I also contacted state residential schools for students with visual impairments because many students participating in post-high school transition programs also attend the local community college as part of their transition from secondary school to adulthood. As a result, I worked with those offices willing to forward the information for the study to all students with visual impairments receiving services at the collegiate level. Although I did not seek to gather a representative sample to generalize the phenomenon studied to all people with visual impairments, I aimed to gather a representative sample of participants. In addition, I utilized social media and listservs to recruit participants. Unfortunately, centers for independent living did not respond to my solicitation efforts. As a result, I implemented snowball sampling (Patton, 2002) by asking already-enrolled participants to identify other potential participants. 
Please see Appendix A for the solicitation email I sent to institutions. My final sample included seven young adults with visual impairments and six family members, totaling 13 participants.

\section{Methodology}

My methodology is rooted in naturalistic inquiry; specifically, I performed a basic interpretivist study. Naturalistic inquiry, as described by Lincoln and Guba (1985), "is an approach to understanding the social world in which the researcher observes, describes, and interprets the experiences and actions of specific people and groups in societal and cultural context" (p. 880). The naturalistic researcher chooses a specific group of people because they offer a compelling example of a particular phenomenon and the researcher does not seek to find a representative case from which to generalize findings. The interpretivist qualitative researcher is interested in learning and representing participants' experiences, perceptions, and points of view. Naturalistic inquiry designs are particularly worthwhile and productive for exploratory stages of research, especially when little is known about the phenomenon to be investigated.

Major characteristics of naturalistic research are: (a) researchers develop interpretations and theories that afford insight into the human experience of a specified population, (b) research is conducted in the field, (c) researchers spend large amounts of time interacting directly with participants, (d) researchers learn through all of their senses, (e) researchers modify their methodological strategies throughout the research process to obtain data that will bring to light more focused or intriguing questions, and (e) the process is circular, with observations and interviews informing the researcher and leading to more improved observations and interpretations (Lincoln \& Guba, 1985). Purposive samples are also utilized meaning participants are chosen based on their membership to the group or phenomenon under investigation. I captured each of these components by first reaching out to a specific subsection of people with 
visual impairments because this population is who experiences the phenomenon. Next, I observed people with LV/B traveling in familiar and unfamiliar environments. This provided useful information regarding their independent travel skills, which I used to inform interview questions and to determine if their perceived O\&M abilities were, in fact, accurate. Next, I communicated with participants through interviews and follow-up interviews. The best way to learn about a person's experience is to ask him about it. I established a rapport and, over time, made participants comfortable enough to provide open, honest, and lengthy responses. This provided a plethora of data of which I utilized to continuously modify theory and methodology. While I interviewed and observed participants, I took descriptive field notes rich with sensory information. I want the reader to experience the participant's world from my perspective so she can better understand the significance of the phenomenon I am studying.

Naturalistic researchers conducting field research go through a common sequence of steps: (a) gaining access to and entering the field site, (b) gathering data, (c) verifying and crosschecking findings to ensure accuracy and trustworthiness, (d) analyzing data immediately and throughout the study, (e) making interpretations, (f) writing up findings, (g) sharing conclusions and conferring with participants, and (h) leaving the field site (Lincoln \& Guba, 1985). I followed these similar steps during the study by collecting artifacts, taking field notes of observations, and interviewing participants. After each event I transcribed the experience using rich sensory detail. Then, I examined and reexamined the data collected to determine common or interesting themes across the various data collection methods. I repeated this cycle after each data collection session. Throughout this process, I conferred with participants to ensure the findings are an accurate representation of their experiences. 
In addition to the cycle of steps performed by a naturalistic researcher, it is also important that she evaluates the quality of her work. I do this by becoming well acquainted with the field site through multiple observations; by being transparent with the reader and disclosing my biases, subjectivities, and data; ensuring that my information is accurate through interviews with participants and their parents/guardians; by protecting participants from harm through confidentiality of their personal information since it is possible they will be disclosing private thoughts and feelings; and ensuring reciprocity for participants (Lincoln \& Guba, 1985). Although there is a common sequence of steps, naturalistic inquiry is nothing if not flexible and fluid when it comes to what methods are implemented when and in what order. Researchers use the data they collect from observations and interviews to inform future observations and interviews. They tend to "bounce between" steps to explore the most salient themes directly as they emerge, grounding their decision-making regarding methods in formative data analysis.

Naturalistic inquiry is a subjective enterprise. Objectivity and detachment are thought neither to be possible nor desirable. The researcher's subjective experiences act as a source of data that can afford him the understanding of the social phenomenon in question through his experience with empathy and subjectivity (Lincoln \& Guba, 1985) as well as a passion for the phenomenon under study. With this methodology or philosophy of method as the foundation of my study, I next describe the methods or tools appropriate to naturalistic inquiry, explaining and justifying my choices for what I utilized to carry out this research.

\section{Methods}

The research methods I employ in this study are participant observation, interviews, and artifact/document collection. Observations took place not just in interview settings but also during times when participants engaged in independent travel, attended class, and walked to 
work. This is not a case study because I am working broadly to define a phenomenon. I am not able to determine what is typical for this phenomenon until I conduct this foundational work. After conducting this study, a case study can be identified that acts as a good example of some aspect of this broader phenomenon, but not before this definitional work is completed. In addition, this is not a comparative analysis because I have not defined the phenomenon and before I am able to compare or show differences, the phenomenon must be defined. Instead, I use inductive analysis to identify themes emerging from interviews, document analyses, and openended observations. All participant interviews were conducted in person so I could observe behaviors and settings during interviews.

Participants with visual impairments who met the exclusionary criteria for participation in this study were interviewed for a minimum of one session. During the first session, I explored the subject's educational history, experiences with O\&M, family attitudes, and her feelings about driver's education (for a full list of interview questions, please see Appendix H). Post-interview, I transcribed the audio from the interview and determined follow-up questions. A second interview was scheduled, when appropriate, to expand on the follow-up questions devised from the initial interview or to clarify information from the initial interview and observation. If follow-up questions were only for clarification and would not have elicited a storied response, a follow-up interview was not scheduled. Thirteen initial interviews and three follow-up interviews were conducted for a total of 16 interviews. I arranged to observe all seven participants at work, in class, or engaged in some other activity. All participants lived within a day's drive from my home. I also conducted interviews with at least one parent/guardian of participants to provide context for the young adults' experience of driver's education through educational policy, attitude, and pedagogical practice. 
In terms of order of events, it was often more feasible to interview participants before observing them. Participants were outgoing and made it easy to establish a rapport and increased comfort before asking personal questions. During observations, I was an active observer, asking questions as they arose and referred to responses from the initial interview when asking questions during the observation. Due to the availability of participants, the distance to travel to get to participants, and follow-up questions being asked during the observation, many participants only engaged in an initial structured interview.

\section{Methods of Analysis}

I analyzed data using the constant comparative analysis method. The constant comparative method includes both coding and analysis simultaneously to generate theory (Glaser $\&$ Strauss, 2012). In this method, theory is constantly being designed and redesigned, according to the researcher's interpretations of the data. The constant comparative method is used when the researcher intends to "generate and plausibly suggest many categories, properties, and hypotheses about general problems" (Glaser \& Strauss, 2012, p. 104). Researchers using the constant comparative method are not looking for universality or proof of causality. Instead, they look for a saturation of data to determine which themes emerge instead of considering all of the available data. As a naturalistic inquirer, I use the constant comparative method when collecting and analyzing data because it allows me the flexibility necessary to define the phenomenon.

\section{Theoretical Framework for Data Analysis}

The theoretical framework I proposed at the beginning of this process was drawn from the resilience theory of HeavyRunner and Marshall (2003). As data were gathered and examined, I found that, on its own, resilience theory did not fully encapsulate the richness of the data to be explored. While it invokes the value of community and family in promoting resilience in 
marginalized populations and draws attention to the ways in which the individual can demonstrate resilience, it is limited in its ability to prescribe societal intervention or to explain the complexity of the politics the data revealed for emancipating these groups. By enlisting Kim's (2015) theory of unbecoming human and the ways in which people with disabilities are treated as "less-than," I am able to analyze a greater portion of the data by looking at the implications of societal perceptions of incapacity based on disability characteristics and the effects of this perspective on self-efficacy, identity construction, and transition for young adults with visual impairments.

How people view themselves affects what they are willing to attempt and the goals they set for themselves. In the following, I provide the history of the development of resilience theory and the ways in which it is used to analyze the data as an important component of my theoretical framework. Then I close by defining Kim's (2015) theory of unbecoming human and explaining how I used her concept to deepen data analysis to reveal society's use of agency, utility, and capacity to oppress people with disabilities.

\section{Theoretical Concepts Defined}

The concepts and subconcepts that compose my theoretical framework all share a relation to identity, by which I mean, the internal and external factors that play a role in a person's membership to a specific social category and/or a distinguishing feature someone takes special pride in (Fearon, 1999; Forber-Pratt \& Zape, 2017). I have chosen concepts I see as complimenting and magnifying identity because data from interviews and observations point to ways in which participants construct and perform their identities. I analyze my data using the following theoretical concepts as my lens: significant adversity and positive adaptation to adversity, which I draw from resilience theory (HeavyRunner \& Marshall, 2003), and the 
concept of unbecoming human, specifically the notions of object-becoming and proximity (Kim, 2015). These concepts are rooted in emancipatory social theories developed by HeavyRunner and Marshall (2003) and Kim (2015). I choose these concepts through which to analyze my data because young adults with visual impairments are among a number of marginalized groups who continue to experience societal discrimination, even in an age of legislation that promotes inclusion of people with disabilities. These three theorists suggest a shift in discourse from deficit thinking to strengths-based ontologies to affect positive social change for Native American cultures and people with disabilities. This work is consistent with the work of Yosso (2005), who first conceptualized community cultural wealth as a means to illuminate the strengths-based perspective of the value of "cultural knowledge, skills, abilities, and contacts held by socially marginalized groups" (p. 69), rooted in race-based oppression that have gone unrecognized and unacknowledged as containing significance. One of the hallmarks of HeavyRunner and Marshall's (2003) theory is their use of the term "human" as a means to include all people. As members of the "human race," HeavyRunner and Marshall (2003) reverse the idea that Native Americans are subhuman and therefore, undeserving of the same treatment as whites. It also empowers the individual by highlighting his capacity and value, with the potential positively to affect community and individual identity through inclusivity. Kim (2015), however, describes how the term "human" has been used to marginalize categories of people and suggests "unbecoming human" to challenge the discrimination and oppression of people with disabilities as a result of "missing" abilities. She offers unbecoming human as a societal intervention to the status quo by suspending what society understands as qualities that are distinctly "human" (and thus used to oppress) to remove the societal value placed on ability, capacity, and agency as means to measure "humanness." HeavyRunner and Marshall (2003) 
recommend resilience theory as an intervention to empower the individual to construct a healthy identity by including him as "human" while Kim (2015) asks society to ignore the ways in which it measures humanity to address the societal oppression and treatment of deprecated groups.

History of resilience theory. My chosen frame is pertinent to the issue of nondriving and driver's education because culture and family play a vital role in a person's desire to be independent and how people with visual impairments respond to adversity. Self-determination, as noted in my review of the literature, is important to develop for a person truly to realize independence (Sheffield, 2014) and it is necessary for a person to understand how he thinks and feels in order to evaluate options and choices to make life decisions, including those for transition to adulthood. Resilience theory is in a similar vein to self-determination because it requires a person to understand how she thinks and feels coupled with the positive response to adversity to demonstrate resilience. Resilience theory arises from research concerning underrepresented and marginalized groups, most notably Native Americans (HeavyRunner \& Marshall, 2003) and other indigenous groups across the world because these are communities who have been stripped of their collective identities in order to assimilate them into the settlercolonized culture and this theory is a means by which they offer an alternative to the identity of "brokenness," "lacking," and "outsider." In the early years of resilience studies, focus was on the individual as the source of resilience. It was his innate capacity to rise up against adversity that characterized a person's resiliency. As resilience theory evolved, researchers became more interested in the social dimensions of resilience (family, community, and culture; Fleming \& Ledogar, 2008). As noted by Fleming and Ledogar (2008), resilience can develop over time and with the help of family members promoting positive adaptation despite adversity. An additional point of interest described by Luthar (2006) is that resiliency is not an "across-the-board 
phenomenon" occurrence (p. 741). Some people might be resilient educationally but not socially or emotionally. This is important to note because it shows the complexity of resilience and that it is not a discrete state of being that exists in absolutes or dichotomies; it is fluid and everchanging/evolving.

Definition of resilience. According to HeavyRunner and Marshall (2003), "resilience is the natural, human capacity to navigate life well" (p. 14). The word "human" in their definition is important because it includes humans as a species, not just those who are members of a particular social category or who take special pride in a feature that distinguishes them socially that aligns with the majority culture. They advocate for a proactive perspective that gives power to the individual and theirs is a perspective that defines resilience in a way that is inclusive, not exclusive; an issue that has marginalized Native American populations for centuries. They argue that "learning how to utilize innate resilience" is each person's birthright (p. 14), important because it reminds native populations they have the capacity to overcome significant adversity in a positive way even though the majority culture tells them otherwise and has labeled them "damaged goods" (p. 15).

Resilience includes wisdom and common sense and is measured by a person's ability to know how she thinks, who she is spiritually, where she comes from, and where she is going (2003). This perspective is important to native populations because their knowledge and skills have long been devalued and referred to as "savage" in comparison to the "civilized" white man (White, 1985). To reclaim an identity as wise and beholding common sense is a pivotal step in theorizing their existence as it runs in opposition to what they have been told for ages. Resilience theory incorporates culture and family, positive response to adversity, and a person's ability to understand how she feels and thinks. In the case of Native American culture, family is not 
limited to nuclear family members, a post-WWII, U.S. invention (e.g. mother, father, sister, brother); it extends much further to include blood and non-blood relationships in the community. Family is extremely important to resilience theory because it is one of the main sources from which people learn the skills to be resilient. To what capacity a person demonstrates resilience depends on how he has learned to utilize it. Resilience theory is understanding that the potential exists in an individual to be resilient in the face of hardship, it is the responsibility of the person, family, and community to teach the individual to access this capacity to overcome significantly adverse situations. Characterization as "human," wisdom, common sense, and resilience, when present, are components that lead a person to develop a positive sense of self and establish a healthy identity.

Essentialism is the idea that certain traits are innate, static, and something with which a person is born, not something constructed by society. It is also a philosophy that has allowed for discrimination and oppression based on the privileging of specific traits above others. One of the ways essentialism has been used to promote some above others in society is under the guise of scientific objectivity, using the "natural order" to evidence the inferiority of beings who possess certain physical, intellectual, and emotional qualities that differ from those in a position of power. Resilience theory lays bare the danger of falling into an essentialist way of looking at groups of people and revels the logical problem with essentialism. This theory is emancipatory in its intent and anti-essentialist in nature because it is rooted in philosophy that resilience is indeed a social, ideological, and intellectual construct that can and should be promoted by the individual, family, and community. While resilience theory is useful in analyzing some of the multiple dimensions of identity construction by the individual, I look to Kim's (2015) theory of unbecoming human to examine ableist discrimination at a societal level. 
Unbecoming human. Eunjung Kim is an activist and cultural theorist in the areas of feminism, asexuality, and disabilities. According to Kim (2015), the terms "humanity" and "humanness" have been used to exclude people who do not fit the definitions of these terms, thus creating groups of people who are othered. The distinction between "human" and "inhuman" or "normal" and "abnormal" are at the heart of the "civilized" vs. "savage" debate (White, 1985) and is the bedrock that holds up slavery, colonialism, genocide, and most forms of oppression and evil. This perceived lack of humanity based on differences provided settlers the opportunity to impose their will as an excuse to "usher these individuals into modernity and humanity through education," (Kim, 2015, p. 306). Education, however, is used to "normalize" settled populations as a means to create docile bodies (Foucault, 1977) which are easier to control and regulate. Those assigned for "normalization" have been labeled "savage" or "unhuman." This form of education is rooted in brutality: removing identity, removing family and culture, torture, cultural genocide, and forcing people physically to conform to the values and practices of the oppressor. Thomas Morgan, Indian Commissioner, exposed the true motivation for educating Native Americans through forced assimilation in Indian Boarding Schools in the establishment of the Phoenix Indian School in 1891, "It's cheaper to educate Indians than to kill them" (Lindauer, 1998). Within the historical context of the oppressive treatment of Native Americans, resilience theory is even more potent in its effort to reclaim the identities of those who have been stripped and othered time and time again. The "human" vs. "inhuman" debate is also used as a way of separating those worthy of life, freedom, and entitled to resources from those who are not. This dichotomy often translates to characterizations of people with disabilities.

The differentiation made between humans and objects is that humans are not objects because they have the ability to see and know (Dworkin, 1981), whereas mere objects lack these 
capabilities. Kim (2015) explains that much value is placed on ability, competence, agency, and dignity and the presence (or absence) of these characteristics is used to define a person as human or inhuman. The belief persists that normativity is equal to humanness and that non-normativity is equal to nonhumanness and illegitimacy and makes those who are in the normative group believe they are justified in treating those outside their group as less-than-human and that the non-normative should not be guaranteed the same rights or even basic human rights. This is evidenced by Kim's reference to an experience of Ed Roberts, a disability activist who was paralyzed from the neck down after contracting polio as a teenager. Mr. Roberts recounts his experience when a doctor told his mother, "You should hope he dies, because if he lives, he'll be no more than a vegetable for the rest of his life. How would you [emphasis added] like to live in an iron lung 24 hours a day?" (Roberts, 1977, p. 1). The groups Kim refers to who have been denied rights and labeled less-than-human are members of specific racial, gender, class, ability, and sexuality groups that fall outside the normative group. These include men and women of color, homosexuals, and people with disabilities. The consequences of such an ability to characterize people in this way has the potential to result in severe violations of human rights and is the argument used to rationalize genocide in recent history.

In Kim's argument, Ed Roberts lost or relinquished his position as subject, becoming viewed as an object due to his inability physically to engage with his environment in the way "able" bodies can. While comparing people to objects is viewed as offensive, Kim (2015) boldly suggests we change the way we define "humanness," and thus, how we consider objects, because this prior definition has been used purposefully as a way to cast out those who do not meet the narrow criteria of "humanity." She uses examples of works where people intentionally become objects to demonstrate the frailty of the human/object line and to suspend what are typically 
viewed as "uniquely human capacities and values" (p. 295) to posit an alternative to what she refers to as an ableist perspective. Through her analysis of normativity in society, she theorizes ways that would allow society to strip itself of judging others by ability, agency and difference. Her major theoretical contribution is the notion of "unbecoming human." To unbecome human is to blur the line between human-being and object-being to investigate a "theoretical and ethical intervention" (Kim, 2015, p. 304) that may act as an alternative to values, legitimacy, abilities, and social acceptance as a means to determine "humanness" and, therefore, worth. This move is significant because it offers a remedy to the pervasive nature of discrimination by suspending value placed on the characteristics that purposefully place people with disabilities "outside" the norm. Kim (2015) calls for us to consider unbecoming human in response to the social order of societal domination in order to emancipate people with disabilities from the shackles of perceived incapacity because it requires us to reject the desire for normativity, one of the tools used to dominate.

Kim theorizes one way to "unbecome human" is through "object becoming," characterized by humans' proximity to objecthood. Objects do not contain agency and are powerless. By engaging in acts of surrendering agency and practicing powerlessness, we relinquish the value, and thus, control that comes from attempting to achieve these markers of humanity. The idea of objectification seems to defy logic because it has been used to shine a light on serious problems for those who have been othered (Kim, 2015), however, this viewpoint neglects the myriad of ways in which objects create meaning. Objects contain different meanings depending on the context within which they exist. In this way, a narrative can be created based on the object that is absent of the valuation of productivity, utility, and supposed ablebodiedness. By viewing human bodies as objects, theoretically we surrender the power to inflict 
pain (because objects do not feel pain) and relate to each other through violence. In object becoming, Kim (2015) suggests an "ethical and theoretical intervention" that departs from value placed on agency, legitimacy, and ability used to separate the deserving from the undeserving. While Kim (2015) does not address the problem of essentialism, it underlies her theory of unbecoming human. This is a way in which these two different theoretical concepts from two different disciplines work together to shed different types of light on my data.

\section{Methodological Roots and Formative-Analysis-Based Design Changes}

When I set about to answer the question, "What is the perceived value of driver's education to nondrivers with visual impairments?," I neither expected to grow in the ways I did through the process of conducting a naturalistic research study, nor did I understand fully the meaning of the methodological term "emergent," a central philosophical principle of qualitative research design and analysis. I learned, through this experience, that interpretivist, naturalistic qualitative inquiry is a fluid and circular philosophy of method and methodological process that requires a researcher to embrace flexibility as the study progresses. As a result, methodological steps are thoughtfully designed and revised using insight revealed by formative data analysis. Such revision occurs throughout the data collection, organization, and analysis stages. While there may be a typical order in which the qualitative researcher completes methodological steps, she may revisit previous steps as deemed necessary and logical; there is no "rule" that limits her ability to collect additional data in the form of interviews, observations, or artifacts if formative data analysis raises further questions, as thoughtful data analysis should, leading to "improved observations and interpretations to foster even better questions" (Armstrong, 2010, p. 881). In terms of my growth as a researcher, two very distinct changes occurred: (a) my methodology and methods changed to accommodate the emergent nature of qualitative inquiry in response to what 
I was hearing and seeing in the field by using the constant comparative method of formative analysis; and (b) my theoretical frame shifted, broadened, and deepened based on formative and summative data analysis and in conversations with my co-chair/methodologist. Making my theoretical frame more complex allowed me to address the kinds of patterns I was seeing in my data, particularly with regard to inequities participants experienced and given participants' commitment to activism and advocacy. I first address the change in my methodology and methods.

The impetus of this study was rooted in my experience as a TVI/COMS in PK-12 schools. Although I designed an interpretivist study, setting out to draw from my participants' perceptions and experiences and then utilize theory to analyze to my data, I found myself trapped and narrowed mentally by the concrete nature of my career as a practitioner, not broadened by my knowledge as a qualitative researcher. As a result, I found myself solely focusing on participants' perceptions and experiences of impairments to driver's education during formative data analysis rather than allowing what participants were trying to communicate emerge, notably the role of nondriving in identity construction and transition rites to adulthood. An integral step of interpretivist qualitative data analysis is for the researcher purposefully to let go of the research question, interview questions, and preconceived ideas of what is in the data and, instead, purposively to look at the data without preconceptions so the researcher can hear what participants are trying to communicate, not what the researcher sets out to find or what the researcher chooses to hear based on her research questions (Otto \& Gunzenhauser, 2006; Otto \& Gunzenhauser, 2014). By letting go of the researcher's focus of inquiry, the research question, the researcher opens him or herself to "the more" (Worley, Otto, \& Bailey, 2010; Otto, 2007) within qualitative data in alignment with the ethical tenets of interpretivist and emancipatory 
qualitative inquiry, honoring what participants set out to communicate. When the researcher lets go of what he or she "thinks is there," then inductive data analysis may more effectively be utilized to "allow the important analysis dimensions to emerge from patterns found in the cases under study without presupposing in advance what the important dimensions will be" (Patton, 2002, p. 56).

Additionally, the constant comparative method of qualitative data analysis allows further flexibility because it includes simultaneous coding (or data organization) and data analysis (Glaser \& Strauss, 2012), which are the emergent aspects of data analysis at the heart of interpretivist practice. Without looking at the data with fresh eyes and letting go of preconceived ideas of meaning, I may not have discovered participants' perceptions and experiences of underlying issues to driver's education were wrapped up in identity construction, development of self-efficacy, and the effects of these on their transitions to adulthood.

Through looking at the data with fresh eyes and through conversations with my cochair/methodologist, participant interviews, observations, and collected artifacts clearly communicated an overarching schema of identity and themes under that schema which related to participants' creation of identity, self-efficacy, and transition to adulthood. Since one never reports all data in any study write-up, a pivotal first step occurred when we made the decision to center identity and to expand upon themes relating to identity as the primary focus of my data analysis and results reporting.

After naming "identity" as the dissertation's schema, deliberate choices were then made about what themes I would address and what data to include in order to construct the analytic claims that would make up my argument. Had it not been for this important decision to focus on participants' experiences of and perceptions around identity, I likely would not have come to 
incorporate Kim's (2015) theory of "unbecoming human" because I would not have made the connection between the data and her theory that critically addresses ableist perspectives and the damage that can result in terms of identity and self-efficacy. This shift called for me creating a far more complex, interdisciplinary theoretical framework than originally proposed through which to analyze my data.

In my dissertation proposal, I planned to utilize Heavyrunner and Marshall's (2003) resilience theory as the theoretical lens through which to analyze my data because indigenous populations and some disability categories gravitate toward a collectivist orientation, meaning the success of the individual is equal to the success of the community. By familiarizing myself with resilience theory, I observed parallels between the collectivist nature of indigenous populations and young adults with visual impairments. The idea it "takes a village to raise a child" refers to how one's family and community provide support and training for the member with a disability to mitigate the potentially limiting effects of the disability, such as how the nature of one's impairment may require an individual to rely on others for everyday tasks such as transportation to a destination, orientation to a new environment, or accessing the printed word. Additionally, I noticed compelling similarities between the oppression of indigenous people and the oppression of people with visual impairments and believed this theory would allow me to analyze my data through a lens that matched philosophically and sociopolitically with participants' perceptions and experiences.

Consistent with interpretivist qualitative research, data collection during this study was, by design, a formative process that resulted in emergent methods and protocol, requiring three versions of the participant interview protocol and three versions of the family member interview protocol based on formative data analysis of already-collected data. Consequently, after I 
conducted two initial participant interviews, I revised questions based on interviewee responses to illicit more storied responses and, in the case of family member interviews, designed to end the interview on a less-emotionally charged note (see Appendix I interview questions).

Once data was collected and transcribed, I coded line by line, taking note of similarities and patterns across participant interviews, observational data, and artifacts. As an interpretivist, in my initial attempt to build analytic claims using resilience theory as my theoretical framework, I needed to avoid pairing the data with the theory to support the notion that resilience is a prerequisite to successful transition to adulthood for youth with visual impairments; simply comparing data to theoretical concepts to determine whether the data supports the theory is not analysis. As expected, as my study progressed, formative analysis exposed resilience theory to be too limiting as the sole brick on which my theoretical frame was built. Whereas resilience theory highlights the value and importance of family and community in promoting resilience among marginalized populations and how these oppressed peoples demonstrate resilience, it does not encompass the complexity of themes that emerged during data coding: driving as a rite of passage; identity construction and the role of ableism and ableist perspectives in this construction; the inability to drive and how it may affect identity construction; and orientation and mobility instruction as a means to construct an identity as a competent, independent traveler. One of the benefits of the constant comparative method of analysis is it focuses the researcher primarily on formative analysis, allowing the researcher to be flexible as he or she learns more deeply about the participants and phenomenon, designing and redesigning methods according to data interpretations. Since interpretivist qualitative researchers make no claim to generalizability, social theory is employed as a frame through which to analyze data so analytic findings can have greater meaning beyond the localized meanings of participants in their particular setting. When 
an interpretivist study is designed, a theory that appears to intersect with the research and interview questions is used as a "placeholder." Resilience theory was the proposed placeholder in my study. As the interpretivist researcher conducts formative analysis and learns from her participants, it is likely the original theoretical frame placeholder will no longer be suitable or will need to be enriched and made more complex to accommodate the complexity of the data. Theoretical frames are built out of different theoretical concepts, often drawing theoretical concepts from a variety of theorists, and, oftentimes, different disciplines. Since resilience theory left a large, central focus of my data unexplored or explained, my methodologist/co-chair suggested I explore Eunjung Kim's (2015) Unbecoming Human, a critical, emancipatory theory focused on disability, potentially to expand my theoretical frame because Kim's work critically theorizes ableist perspectives and the damage that results to the identity of those with disabilities. Kim (2015) describes her theoretical concept of "unbecoming human" as a potentially emancipatory means of intervention and worldview to oppressive, ableist mentalities that base an individual's legitimacy on his or her ability or inability to fit the norm. Seeing the complexity with which identity emerged as a recurring theme and connecting my data to a key concept in Kim's (2015) broader critical theory of intervention, I reexamined my data for evidence of how people with disabilities construct, expand, and limit their identities. Through the process of reading Unbecoming Human and working to construct the theoretical definition I would employ of the term "identity," I began to shift my original research question sharply toward what participants were telling me, as is consistent with interpretivist qualitative research methodologies: even in an era of anti-discrimination legislation and education, people with disabilities continue to experience significant subtle and overt discrimination based on oppressive ableist perspectives, the results of which have lasting effects on identity construction, 
self-determination, and participants' eventual transition to adulthood and driving/nondriving acts, which often serve as proxies for ableism and adulthood.

Critical social theory is not typically utilized in low vision and blindness research or in the larger field of special education. Special education research tends to focus more on singlestudy subjects, evidence-based practices, and building models for practice rather than on developing critical, field-specific social theory to analyze phenomena. The lack of critical social theory in the larger field of special education is what makes Kim's so unusual, critical, and provocative a voice. A dearth of critical social theory is a shortcoming especially felt in research concerning $\mathrm{LV} / \mathrm{B}$, as there is scant evidence where findings are applied to a larger, social context, and scant critical, emancipatory research centering systemic oppression. As a result, critical social theory must largely be borrowed from disciplines outside special education to expose and confront systematic oppression seen in qualitative data. One such critical discipline is Critical Disability Studies (wholly separate from Special Education, an interdisciplinary, theoretically complex discipline of divergent views which arose from Sociology and which theorizes disability through sociopolitical and cultural perspectives); another is Visual Culture Studies (a rangey, interdisciplinary realm of scholarship which arose from Art History, and which, among other things, problematizes the nature of seeing and things seen). Researchers and theorists in critical disability studies have much in common with teachers of children with special needs in that they focus on inclusivity and empowerment, however, critical disability studies scholars operate on macro, sociocultural and sociopolitical levels, whereas special education researchers tend to operate on a much more individually based, more medicalized disability category. Another stark difference between the fields of special education and critical disability studies is scholars of critical disability studies seek to disrupt the discourse regarding 
perceptions of the able-bodied population toward people with disabilities by designing critical social theory that incorporates the reimagining of social ritual, design, and cultural representations of people with disabilities and pushes back against the dominance of "normalcy" (Albrecht, Seelman, \& Bury, 2001). Using critical social theory to analyze my study data allowed me, as is consistent with emancipatory, interpretivist research to spring from local, nongeneralizable data analysis to pan out to a broader, societal level to view participants' stories as microcosms of the manifestation of what Kim (2015) theorizes about ableism, and fully to acknowledge and respect my participants' complex perceptions and experiences of disability as well as their commitments to activism.

Once I had a more finely focused theoretical lens through which to examine the data, I recoded/reorganized my data, compiling $\sim 50$ data "chunks" which not only stuck out to me as important, but were data points in which participants told persuasive stories about equity issues arising from systemic oppression. As I reorganized data, I made analytic memos in terms in order to capture thinking on what I thought the data might mean (e.g. desire to overcompensate due to visual disability, building confidence, mourning and acceptance/rejection of visual impairment) and then began to generate inferences (suppositions about the data that may or not be "true"). Armed with the compilation of compelling stories and with the help of my methodologist/co-chair, we used the theoretical concept of "unbecoming human" to draft initial analytic claims that used the theory to reveal something about the data not immediately apparent when reading the data without the theory which explained the gravity and depth of what participants shared with me. My methodologist/co-chair and I saw that Kim's theory allowed me better to "observe, describe, and interpret the experiences and actions" (Lincoln \& Guba, 1985, p. 88) of participants in social and cultural contexts. I then reorganized the data to locate 
provocative stories to support, broaden, or refute the initial claim. I continued on my own by drafting analytic claims about the data and using those to create a table of claims and to align data chunks which evidenced those claims.

Constructing an analytic claim is important in qualitative research because it is the way a writer may use theory to expose something not immediately apparent in a reading of the data. Constructing analytic claims using your theoretical frame, evidencing those claims using data, and showing the importance of what the theory and data together reveal are what leads to findings (Otto \& Gunzenhauser, 2006; Otto \& Gunzenhauser, 2014) in interpretivist qualitative inquiry. Simply coding and naming themes are not data analysis. According to Booth, Colomb, and Williams (2008), a persuasive argument, in this case an analytic claim about empirical data, must be based on "sound reasoning" and supported by "good evidence" (p. 106). When constructing analytic claims, I found places where the data and theoretical concepts intersected. I worked to glean meaning from that which is deeper than the data immediately appears; things that are below the surface and systemic from the theory's sociopolitical perspective. In my particular dissertation, lurking below participants' words and actions are evidence of systemic oppression enacted on participants resulting from their disability. What I aim to accomplish in this dissertation is to use analysis through my theoretical frame to show how this systemic oppression is being enacted on these highly educated, very capable young people and what that does to their identity formation and their ability to transition to adulthood and thinking about their own self-efficacy.

In interpretivist qualitative research, the researcher often makes claims that ask the reader to think about something in a way or combination she has not considered before. The researcher is tasked with showing the reader how the claim is relevant and why it is important for her to 
consider the well-evidenced truth and knowledge claim. As I generated analytic claims and evidenced them with data, I built them around the story I decided to tell in my dissertation. Through the process of data analysis, positivist and post-positivist researchers seek to streamline their data, to simplify and report it. In contrast, interpretivist researchers do the opposite: we seek to complicate the data. By complicating the data, qualitative findings have depth and complexity because we do not explain away anomalies or control for variables, rather we turn to focus on anomalies (Mathison, 1988) as significant. Emancipatory research, also known as critical theory research, is ideologically specific in that it is tied to a wider discourse of theory and power (Crotty, 1998; Otto, 2017). One of the aims of this form of research is to acknowledge and provide the audience with the tools to challenge identified systems of oppression and challenge these discourses in order potentially to inspire activism and to help the oppressed come to exhibit and enact their own agency. In this dissertation, my mission is first to acknowledge and critique the existence of the power which underlies visual disability. The goal of emancipatory work is not to "save" the oppressed, but to give the oppressed the tools potentially to enact their liberation. Giving the oppressed liberatory tools and practicing liberatory pedagogy is grounded firmly in the experience of the oppressed, and, more importantly, in love (Freire, 2015). 


\section{CHAPTER IV: DATA ANALYSIS}

My impetus for this study arises from encountering adolescents during my years as a teacher in public schools who had visual impairments yet were required to take driver's education. I found little research, if any, exists in the field of visual impairments that draws from qualitative data to examine the lived experiences of young adults with visual impairments, specifically in driver's education. Little work has been done in the field of visual impairments that seeks to apply critical theory to understand and theorize on the experiences of youth and young adults with LV/B. Utilizing the theory of HeavyRunner and Marshall (2003) and Kim (2005) have helped me to reveal important analytic insights that have the potential to inform practice at both grassroots and societal levels. In the following sections, I present the reader with five large analytic claims that emerged from analyzing participant data through my theoretical frame. First, I begin by describing the seven participants so the reader may better understand their perspectives and experiences in the analysis.

\section{Participant Descriptions}

\section{Irene}

Irene is a college graduate in her mid-20s who racially identifies as white. She was raised in an upper-middle class home and was diagnosed as an infant with a rare eye condition that resulted in low vision, light sensitivity and colorblindness. Due to the rarity of her visual diagnosis, Irene's parents, a woman from the United States and a man from the Middle East, did not believe they could both be carrying the same recessive gene that would result in their daughter's eye condition, as they grew up on opposite sides of the world. After further testing and investigation, they came to the conclusion that she did, in fact, have achromatopsia. 
Irene grew up with parents whom I characterize as protective, but not stifling. Her parents always promoted independence and there were few things they believed she was unable to do. For example, despite her low vision, she learned to ride a bicycle and did so often in her town. They express their concerns for her safety at night and with drivers because many drivers fail to stop at traffic controls, but their concern is more with Irene who, as a woman travels in a large city at night. Their concern does not stem from a belief Irene lacks the capacity to travel as a result of her visual impairment. Irene describes her father as an immigrant who moved to the United States to pursue higher education. Through his own experiences and upbringing, he adopted the attitude that his children would grow through their own successes and difficulties. Irene describes her mother as very organized, evidencing how over-prepared her mother was when Irene moved to college. Her mother had purchased almost every gadget to help Irene organize every aspect of her dorm. Irene describes her father as "hands-off" and her mother as "hyper-involved." Irene interprets her parents' worry as the worry of a typical family when a child leaves home and does not let their worries keep her from doing what she aims to do either in life or in independent travel.

Irene attended a private university at which she suspects she was the first, and possibly only, person with a visual impairment to attend. To apply and be accepted to a university that is not known for its accessibility services in a town hours away from her home says something about the way in which Irene views herself: as capable of learning new environments and navigating them effectively. This is clear by the way she describes her experience with independent travel: acquiring a position as a resident assistant and engaging in other aspects of university life in much the same ways as her sighted peers. 
She dresses casually in monochromatic colors, making wardrobe selection much easier since she is colorblind. Irene's large eyes and warm smile make her come across as approachable and easy to talk to. She wears a black rain jacket and has dark glasses; she is ready for whatever the weather may have in store as she walks the streets where she lives. Irene carries a backpack filled with items necessary for the day's activities because she is constantly on the move, working on her own projects and supporting those of her friends in a neighboring city. Irene's mother, Annie, shares that Irene is self-sufficient and gets most places by walking or using ridesharing apps, public transportation, or receiving a ride from a family member to the local train station. Annie also tells me she does not see Irene often even though Irene lives at home, because she is occupied with so many projects, some of which keep her out until late at night. Annie describes Irene as a person who "does her own thing" (which is just the way Annie describes herself), going her own way to achieve her goals. Irene works as a freelancer in the arts, which motivates her to learn about and navigate the neighboring city and its neighborhoods and that knowledge and her attitudes have made her a more confident, competent traveler.

Both Irene and Annie describe a supportive upbringing by parents willing to do whatever is necessary to make sure their child's needs are met. For example, Annie and her husband understood after receiving Irene's diagnosis of a visual impairment the importance of her access to public transportation. For this reason, they uprooted and moved their family to a metropolitan area so their daughter could receive the academic and mobility educations she would need to function independently. Irene identifies as a person with a visual impairment and is thankful she grew up in a place with reliable public transportation because she feels it has maximized her independence. 
Irene talks quickly and enthusiastically, recounting her experiences with her visual impairment in high school, college, and describing future career plans. Irene has shown herself to be persistent and hardworking. Her mother describes instances when Irene experienced rejection in school and looked for opportunities outside of that structure to do what she loved: acting. Irene further describes her work ethic when outlining her chaotic college course load, role as a university resident assistant as well as participation in an internship - all in the same semester. Irene is a self-proclaimed social justice advocate, which is evident as she lists the names of plays she has directed or with which she has been involved. She passionately describes her stage directing of plays about LGBT issues and aims to give a voice to other marginalized groups. Her latest directorial project explores the lived experiences of individuals around the globe who live with a specific form of visual impairment in order to communicate to a broad audience the ways in which people with visual impairments have been "othered" in their respective cultures. Irene continues to build her portfolio with the end goal of going to graduate school to obtain a Master of Fine Arts degree.

\section{Maverick}

Maverick is a white college graduate in his mid-20s. He and his younger brother, Nolan, were raised in a middle-class home. When he answers the door, he looks at me out of the corner of his blue-green eyes, maximizing his peripheral vision. His eyes dart around as he takes in my appearance. Maverick has dark blonde hair and he is tall and thin. He speaks quickly and energetically, volunteering personal information about his life and upcoming nuptials as I enter his apartment. The lights in his apartment are low since the part of his eye that detects light and detail is affected, so he sees better in dim lighting conditions. His smart phone talks to him, telling him the time and that someone has sent him a text message. Maverick describes his 
experience with vision loss when he was 15 years old. Unlike other participants, he and his younger brother, Nolan, were not born with a visual impairment. Their condition presented when they were teenagers as a result of a rare, X-linked gene that caused their optic nerves to become pale. Maverick describes this time during his life as one when he was deeply depressed, thinking about how his life would change as a result of his visual impairment and trying to reconcile how much of his world he accessed visually and how it would be vastly different.

As we walk from his apartment to class, he wears dark, wraparound sunglasses. His shoulders are back and his head is up; he appears confident in the route we are walking as he has been traveling it for a semester. Based on my initial observations of how closely Maverick holds his phone to his face and the use of a screen reader, I expect Maverick to carry a white cane and I am surprised he does not. Without solicitation, Maverick mentions that he uses a cane in unfamiliar settings or when he moves to a new location (e.g. when first moving to his college town). He also accesses his environment visually using his peripheral vision since his central vision is affected by his eye condition.

Maverick, and his mother, Mickey, describe the support and love of their immediate and extended family when receiving the diagnosis, despite being thrust into "crisis mode" (talking to doctors and fighting with local school and district administration to provide accessible materials). His parents went so far as to research educational options for Maverick because his educational needs were not being met by his home school. As a result, Maverick and his family decided he would attend the state school for the visually impaired. Through the program at the residential school, Maverick was able to build his confidence in travel and accessing information through his other senses. While he has some remaining functional vision, he has learned he must research as much as he can about an area, including public transportation, in preparation to travel 
to an unfamiliar environment. His mother echoes the sentiment that his education has equipped him to travel independently not just in his community but all over the world and notes she and her husband have worked to instill independence in Maverick and his younger brother, Nolan, since their boys first became visually impaired. When the boys were young they would ask Mickey to read assignments for them, but she reminded them to use the technology they had available and to problem-solve instead of asking her to do it for them. Mickey felt very strongly that she wanted her sons to do more than "just survive," living on a disability check. She wanted them to thrive and believed that accessing technology and a quality education were the means by which Maverick and Nolan would achieve their goals.

Maverick's diagnosis caused him to change his outlook on life; it is okay to ask for help. He notes that people with vision ask for directions, so it is not abnormal for him to ask for directions also. He has a strong work ethic, which is evidenced by his desire to further his education and by obtaining and maintaining gainful employment for two years while in college. Maverick is employed at his university's disability resource office and despite receiving financial support from his state's department of rehabilitation services to pay for expenses related to his education, he has maintained the same job at the university for two years, gaining communication, organization, and problem-solving skills. He attempted various roles in the resource office, however, his supervisor decided his skills were best suited for the front desk. Maverick is students" first point of contact and "triages" students as they call on the phone or enter the office, deciding which professional to refer them to based on their needs. He greets students with a large smile and takes down phone messages under his video magnifier. He answers students' questions about scheduling final exams and educates them about the process as he also utilizes the services. He is reassuring and speaks softly as he calms panicking students 
navigating the test scheduling process for the first time. In my opinion, he is well-placed in his role at the front desk where he acts as a customer service agent, problem-solver, and social worker for university students with disabilities.

While he believes he has to overcompensate for the perceived shortcomings created by his visual impairment by being extra knowledgeable and extra qualified, this does not discourage him from doing the work necessary to reach his goals, in fact, it strengthens his resolve. As a result, Maverick plans to attend graduate school so he can improve his marketability when he searches for jobs in the near future. He believes that through his use of technology and his education, he will be able to mitigate the potentially limiting aspects of his visual impairment to obtain a job in the field of his choosing.

\section{Nolan}

Nolan is a white college student in his mid-20s who comes from a middle-class family. He has the same eye condition as his brother, Maverick, however, his presented when he was 14 years old. Nolan walks with his head high and shoulders back, appearing confident in his strides. He is tall and thin with short, delicately coiffed blonde hair and crystal blue eyes. As he sits in front of me for the interview, I am enveloped by the scent of laundry soap and cologne. He makes jokes and small talk; however, he does not elaborate much when asked questions about his visual impairment. When pressed further, he shifts in his chair and speaks quickly and in a lower tone as he describes some of the more painful rejections he has experienced because of others realizing he has a visual impairment. As a result, he does not often share his visual impairment with others until he has built a certain level of trust with the individual. Instead, he seeks to "pass" as the majority culture (sighted), a phenomenon that has previously been linked to disguising race (Brune \& Wilson, 2013). This can be especially true for people with low 
vision because they live in the gray; people often do not understand vision outside of the dichotomy of sighted or blind, which is often mischaracterized as total darkness. Since vision loss is different for each individual, it is difficult for the general public to grasp what Nolan and others with low vision do or do not see. Add to the variability in visual functioning the stigma carried by having a disability as not just being inferior in one area, but globally, and you end up with a person who feels forced into hiding his visual impairment, or "passing," to avoid discrimination. To further support this, Nolan describes moments of frustration when people lack understanding concerning his low vision but mentions that this is the lot he was cast and that he does not want to let it affect his life.

Because his brother Maverick experienced the same form of vision loss two years prior, when Nolan lost his vision, his family decided he would also attend the state school for the visually impaired because his home school district did not have the resources to meet Nolan's educational needs. Although Nolan's mother, Mickey, describes this time as "heartbreaking and difficult" because she was sending her boys away from home far earlier than she had expected, she and his father agreed it was the best decision for both their boys.

Nolan's story reveals him to be both hardworking and persistent. He describes some difficulty with his initial attempt at college, he struggled with drinking and partying and did not take attendance to classes seriously. As a result, Nolan lost his financial aid and had to drop out of school. This experience did not dissuade him from continuing on his path to becoming a teacher. While at a day camp specifically for young children with visual impairments, boys and girls run up to Nolan excitedly, shouting his name. A wide smile comes across his face as he leans down to talk to them individually. They tug on his arms and beg him to play and he obliges, galloping alongside them as they rush to get toys. Although he describes experiences 
that have resulted in him having a rough exterior (e.g. a friend ditching him after finding out he was visually impaired), after observing Nolan as he works with children, I can see he is compassionate, warmhearted, and excited to begin his career as a teacher. He credits his instruction at the residential school for the visually impaired for helping him acquire the knowledge and skills that have allowed him to succeed in his professional and educational life.

\section{Devon}

Devon is a middle-class college graduate in his late-20s. He identifies racially as white even though he has dark brown eyes and dark, brown-black hair and stubble on his olive-skinned face. He lives with his mother in an apartment complex in a medium-sized suburb outside of a large city. When I meet him, Devon is wearing a black t-shirt, a round belly hanging out over black shorts, black flip-flops, and his nails are bitten to the quick. He speaks slowly and often replies with "It's cool" or "No worries," making me feel relaxed and as though not much concerns him. Devon notes he and his younger sister were adopted as infants from a country in eastern Europe by white U.S. parents. Devon was diagnosed with low vision, in part, due to complications during the birth process. In the country where he was born, they did not have knowledge of Cesarean-section births, thus, the doctor pulled Devon out using forceps, which dramatically altered the shape of his head and resulted in nerve and facial tissue damage. Although it is believed Devon was born with a visual impairment, it is believed nerve damage is linked to the additional restrictions in his vision. During his PK-12 education, Devon had a stable visual acuity of approximately 20/70 (what a typically sighted person can see from 70 feet, Devon needs to be 20 feet from the same object to see it with the same clarity). At 23 years old, he experienced a deterioration in his visual functioning, resulting in a reduction in acuity that qualifies him as "legally blind" (20/200 in the better eye with best spectacle correction). In the 
last year he began using a long white cane and receiving orientation and mobility training to aid with locating obstacles and wayfinding. He expects his vision further to decline and while the prospect causes him some anxiety, he tempers it with his laid-back attitude and is attempting to prepare for the transition by learning braille, using screen-reading and screen-enlargement software to access his computer and cell phone, and completing orientation and mobility training.

During his youth, Devon's mother, Dora, and father were supportive of Devon's desire to play percussion in a large, traveling competition band. Dora shares that even though nervous about Devon being away from home, she feared imposing her fears on him, potentially stunting his journey to independence and mobility. Dora describes Devon as fiercely independent (a characterization he shares of himself) and resourceful; he conducts the majority of his travel through walking or arranging his own rides from friends, ride-sharing companies, or taxis because the fixed-route bus system only picks up sporadically in his town en route to the neighboring city. He does not frequently ask for rides from others because he maintains that by asking for others to drive him, he is at risk of becoming a burden on those closest to him. Dora expresses pride in Devon's inclination toward independence and resourcefulness when noting he attended college in a large city and taught himself the public transportation system.

During high school, Devon elected to take driver's education because he thought he would be able to drive. When he failed the vision exam, the went to his eye doctor who cleared him to participate in both the classroom and behind-the-wheel portions of the course. Devon earned his learning permit and during this stage, self-selected to stop pursuing a driver's license because his visual impairment caused him to fear for his own safety and the safety of others. 
Currently, Devon utilizes Google Maps to establish and follow routes from one place to another, however, he is aware the system does not always send him on the most "pedestrianfriendly" routes. He uses his remaining vision and other skills taught during orientation and mobility instruction to determine the presence and absence of vehicles in order to cross streets. Devon proactively wants to be "ahead of the curve" in the event he does lose more vision, which in large part is why he has requested additional disability-specific training through his state's vocational rehabilitation department.

Despite the ongoing changes to Devon's vision, he attributes his optimism about his vision loss to being an active member of the blindness community. This is in large part because he communicates regularly with young adults who are blind and who navigate their environment effectively. Through his work as an aide to a local congressman, Devon was exposed to a blindness advocacy group. As a result, he traveled by air to Washington, D.C. to lobby for issues that affect the blind community. When he talks about his advocacy work, he speaks more quickly and with strong opinions, running in contrast to his typically relaxed demeanor. He currently works part-time with the same blindness advocacy group to market educational programs designed for youth with visual impairments. His goal is to live in a large, metropolitan area because Devon knows first-hand how access to public transportation makes travel much easier

for a person with a visual impairment. He is passionate about government work and wants to use this passion to advocate and further the cause of people with visual impairments.

\section{Amelia}

Amelia is a white, middle-class college student in her early 20s. She was born with cataracts and a physical disability due to complications during her mother's pregnancy. As a result of a mishap during surgery as an infant, one of her eyes is permanently dilated, allowing 
too much light to hit her retina, creating an issue with her ability to use her eyes together and makes her sensitive to light. Amelia is tall with shoulder-length, curly hair and wears colorful framed glasses, which she uses to see information at a distance more clearly. While the glasses do not correct her vision to 20/20, they correct it enough to help her gain additional detail she would miss if she did not wear them. As I observe her at her desk, she holds her smart phone a few inches from her face while she reads the enlarged print. Amelia has low vision but uses a long white cane because she is not always comfortable in unfamiliar surroundings. She describes using the white cane as a way to increase her confidence in travel so she does not constantly have to look at the ground to avoid obstacles. She speaks quickly and matter-of-factly and laughs in short, machine gun bursts as she taps her leg. She volunteers personal information about her life without solicitation and talks about her interest in anime.

Amelia transferred to her state's residential school for the visually impaired during her sophomore year of high school as a result of bullying and lack of educational support at her home-district, public school. During this transition, she was quickly thrust into responsibility; she had to manage her own Attention Deficit Hyperactivity Disorder (ADHD) medication and time without the oversight of her mother, Diana. It is important to note that with reduced vision, it is often easy to misplace items, add to that issues with attention and hyperactivity, and organizing oneself becomes even more of a challenge. For Amelia to manage her time and medication while living away from her mother, she demonstrates the capacity for selfmanagement, a skill that has led to her successful transition to college. She also experienced a growth in self-confidence once she became involved in athletics and other activities while at the school for the visually impaired, something she did not engage in while at her home high school. 
Diana comes across as protective of Amelia, her youngest child and only girl. She describes Amelia as "perfect" and becomes upset when people say they are "sorry" when they learn that Amelia has low vision. Even though Diana is protective of Amelia, she wants her to succeed and work hard like other, able-bodied people. Diana's biggest fears come from knowing about the world and its apparent lack of understanding in regard to her daughter and her disabilities. She observed this ignorance in Amelia's home school from teachers who failed to provide accessible materials and out in the world once when she and Amelia crossed the street and a driver almost hit Amelia, even though she was carrying a white cane. Diana believes Amelia can do what she puts her mind to; her fear lies in the potential for her daughter to be hurt, physically and emotionally.

Despite these parental fears, Diana has allowed Amelia to make her own mistakes and wants her to explore her surroundings to gain a better understanding of her world. For example, Diana describes a time when she stood back and watched as a young Amelia climbed up objects and when she almost face-planted in a game of tug-of-war. Starting in eighth grade, Amelia began to experience social and academic issues because her educational materials were not adapted into large print consistently. Diana did what she could to protect Amelia from the bullies and advocate for her needs at her home school, but to no avail. In tenth grade, Amelia elected to transfer to the state school for the visually impaired because she liked the environment after attending summer camps on that campus. Amelia is aware her mother is nervous about her independent travel (especially her travel abroad), but she does not let it stop her from doing what she wants and aspires to do. As Diana has observed Amelia in independent travel situations, her confidence in her daughter's abilities grows and she has become less afraid for Amelia's safety. 
As noted by Amelia and Diana and after observing Amelia during different travel situations, Amelia is a semi-confident traveler (by which I mean confident in familiar environments but very nervous about unfamiliar environments, yet she nevertheless bravely wants to study abroad), which she attributes to her orientation and mobility training at the state school for the visually impaired. From Amelia's perspective, O\&M opened her mind to what she could do because it gave organization to her world in a way that she had not previously known and likely could not have learned on her own. She recalls spending much of her time in her dorm room when she first moved to the residential school before she started mobility training. After extensive training in O\&M, however, she felt confident to travel to different places on her high school's campus and has since successfully transitioned to a medium-sized public university in an unfamiliar city to study elementary education. Amelia plans to study abroad for a semester, which indicates her confidence in her route and travel planning skills, despite her tentativeness to travel to unfamiliar places.

In addition to confidence when traveling in familiar places, Amelia is organized and has a strong work ethic. During my observation, she arrived to the accessible testing site where she would take her final two hours before the scheduled time so she could maximize her study time and prepare herself mentally to take the exam. Additionally, she describes the many extracurricular activities in which she is involved and how they all relate to her goal of becoming an elementary school teacher. Amelia's plans for life after college include moving to a larger metropolitan area because her home town has limited opportunities for public independent travel, which makes her feel stifled and heavily dependent on others. She recognizes larger cities will likely be necessary to ensure she has access to reliable public transportation. 


\section{Tommy}

Tommy is a white, middle-class high school graduate in his late teens. He and his older brother, Terrence, were born with a visual impairment that was passed down from their mother, Adele. As a result, Tommy is severely nearsighted (he has difficulty viewing information at a distance) and is at an increased risk of his retina (the layer of tissue at the back of the eye responsible for perceiving light and sending messages to the brain) detaching from his eye. This has limited the types of activities in which he can engage. For example, he is not medically permitted to play contact sports because if his retina detaches, he could lose his vision completely. This is an ongoing source of frustration for Tommy because he feels limited by his vision to engage in physical activities. Despite having a degenerative eye condition, his visual acuity (clarity in vision) has remained stable for the majority of his life. When I meet with Tommy, he is wearing a cut-off orange t-shirt and black and blue athletic shorts. He has messy, dark blonde hair and light stubble on his face. He is tall and doughy with thick glasses that look like the bottoms of Coca-Cola bottles. His eyes look tiny through the magnified lenses. He speaks quickly and bounces from one topic of conversation to another. Tommy is friendly and shows he is caring by asking me questions about my personal life in an attempt to get to know me.

Tommy transferred to his state's school for the visually impaired when he was 16 years old. Similar to other participants, he was not receiving the accommodations he needed to succeed academically in public schools and he did not have access to a certified teacher of students with visual impairments to provide support. His older brother, Terrence, also diagnosed with a less severe version of Tommy's visual impairment, attended the school for the visually impaired prior to Tommy's arrival because he experienced similar shortcomings in their town's high school. 
When Tommy transitioned to the residential school, he tells me he had to become much more organized and responsible for himself, a change which carried over into his life when he returned home. He describes learning to set and regulate his bedtime and wake-up time, doing his laundry, and showering regularly without being reminded. Rather than his parents reminding him, he was supported by the school's dorm staff, however, he learned quickly to manage these activities on his own. He also felt more at ease at the school for the visually impaired: his IEP was followed and he did not feel like he had to advocate constantly to receive the accommodations to which he was legally entitled. He also felt a sense of camaraderie and belonging because he was "like everyone else," resulting in improved self-esteem, self-confidence, and empathy for others. He could relate to his peers in a way he could not in his home school and began to support those with less vision than him by offering assistance when he noticed they had difficulty traveling or engaging in everyday activities. This was a noticeable shift from his former attitude of only helping those he liked to having compassion for anyone he came across. Although his sighted peers were engaging in their own rite of passage by obtaining driver's permits and becoming more mobile, he and his classmates with visual impairments were engaging in a different, yet equally important rite of passage from childhood into young adulthood: building self-confidence and self-esteem through learning how to be responsible for themselves and to each other in an effort to be more mobile.

Although Tommy accesses his environment visually, he uses a long white cane at night because he experiences night blindness as a result of his eye condition. During the day, he walks and uses the fixed-route bus system in his town. He has enough functional vision to read the scrolling signs on the front of the buses to differentiate routes. When he attended his home middle school, Tommy took a special education bus from the front door of his home to the front 
door of the school. He asked his mother, Adele, who is also visually impaired, to show him how to take the fixed-route city bus because his public school peers cruelly asked if he was "mentally unstable" or "ill" because he rode the special education bus. Because of the stigma carried by these assumptions, he chose an alternate, independent method of transportation, even if it took longer to arrive at his destination. According to Adele, she only had to teach Tommy the bus route once before he was able to apply the skill to arrive to and from school safely each day. Sadly, Adele has passed away since the completion of this study. She fostered independence in her children by allowing them to engage in the same activities as their sighted peers (e.g. riding a bicycle, walking to friends' houses). She spoke quickly and quietly and asked about my personal life, showing her compassion and care for others. Tommy is a spitting image of Adele in physical appearance and in temperament. Tommy notes Adele was there to support him when necessary and understood some of the nuances and challenges associated with living with a visual impairment.

In addition to being helpful and compassionate, Tommy is intelligent and highly perceptive and demonstrates this by observing the actions of others and through recounting his own experiences. Without any formal instruction, he understands how to apply his knowledge of compass directions to the layout and direction of the streets in his hometown and is able to access the fixed-route bus system in town. This is impressive because these are all skills to engage in high levels of problem-solving typically taught by a Certified Orientation and Mobility Specialist. Tommy explains that he takes the information he learned in public school and from his parents and applies it to independent travel in ways both meaningful and effective for him. Tommy also communicates his visual abilities and needs clearly. He describes what he sees in 
various situations in such a way it is easy for me to understand how he views and navigates the world. For example, he tells me how close he must be to an object to see it clearly. Tommy plans to study computer science at his local community college once he completes the transitional-living program at his state school for the visually impaired. There, he takes community college courses at no cost and also receives the support of a certified teacher of the visually impaired and Certified Orientation and Mobility specialist. If he chose to return to his hometown to attend community college, he would be eligible for mobility services through his state's vocational rehabilitation program, but not the added training and support of staff trained specifically to work with students with visual impairments in additional areas of the expanded core curriculum (e.g. assistive technology, braille, sports and leisure). Another reason he plans to continue with the transitional living program until he is 22 years old is because he has observed and considered the challenges his older brother, Terrence, has had with their area's vocational rehabilitation counselor. After community college, he plans to transfer to the fouryear university in his hometown.

\section{Wolfgang}

Wolfgang is a college graduate in his early-20s. He was brought up in a home that he classifies as "lower-middle-class" and racially identifies as Pakistani with Greek roots. He evidences the importance of his ancestry as he describes his forefathers from Greek tribes who fled into the mountains of Pakistan during Alexander the Great's rule. Over the years, his family members converted from Aryan to Sikhism to Islam, an ideological lineage of which Wolfgang is proud. Today, Wolfgang, his brother, and his mother, Allegra, are all practicing Muslims, an important aspect of their lives. In fact, after our interview concluded I observed Wolfgang turn to face East and give thanks during his afternoon prayer ritual. 
When I first meet Wolfgang, he is carrying a large bag of hockey gear and his long white cane as he enters the library. Wolfgang is a tall, barrel-chested young man with brown skin and long, black, curly hair tucked under a flat-brimmed ball cap. He wears his blind hockey team's jersey because after the interview he will travel by commuter train to practice in a nearby suburb. Wolfgang was born with an eye condition that affects his peripheral vision, but it did not present until he was in fifth grade. His vision is such that, as he has gotten older, his vision on the periphery has caved in on itself, reducing inward to create a tunnel of central vision. His existing visual clarity is compromised enough that he has difficulty discerning fine detail and traveling in dark settings is difficult. Despite having low vision, he is an avid skateboarder and extreme sport enthusiast.

Wolfgang is soft-spoken as he recounts his early educational experiences in PK-12 public schools in the suburbs of a large, Midwestern city. Once he was diagnosed with his visual impairment, he quickly began working with a certified teacher of students with visual impairments to accommodate classroom materials and a Certified Orientation and Mobility Specialist to teach him to cross streets safely and to use a long white cane. He describes his independent travel instruction positively, saying it was fun to use public transportation and go to places of his choosing with the COMS. He also notes the importance planning plays in mobility training in terms of understanding where he is and how to get to his destination. In addition to mobility instruction, Wolfgang attributes his involvement in sports as another means by which he better understands how his body moves in space, which increases his self-confidence when traveling independently.

As a member of the blind hockey team, he has traveled to Canada to play with other athletes with visual impairments. This required him to fly by himself to join the team for a 
tournament. When describing this experience, Wolfgang refers to airport staff as "helpful" and that getting around was "easy." He is also diplomatic when he describes a negative experience in college when his roommates "did not treat him too nicely" because they did not "understand his disability." He did not name call or say mean things about these people, just that they were not nice to him. In addition to showing grace to others who have wronged him, Allegra notes that Wolfgang has told her previously he has thanked Allah he was diagnosed with a visual impairment because he has the inner strength and support network to deal with it. These statements lead me to deduce that Wolfgang is inclined to look at life events in an optimistic way focused on gratitude. His laid-back, soft-spoken, and gentle demeanor runs in stark contrast to his physical appearance. He is tall and appears intimidating by the way he draws his shoulders back and broadens his chest as he walks. Allegra describes Wolfgang's physical appearance and countenance as purposeful: he has grown out his hair and, occasionally, his beard and carries himself in a way to deter people from seeing him as a target while carrying his long white cane. In addition to his gentle nature, Wolfgang is dedicated and hardworking. For example, he was made captain of his hockey team because he arrived at the rink four hours before hockey practice started to ensure he would be there in time for practice. His teammates and coaches observed this behavior and unanimously voted to put him in a leadership role. Additionally, he promotes blind hockey on social media to help the sport grow in the U.S. Hockey has played a large role in building Wolfgang's self-confidence and has been an overall positive experience for him.

Unfortunately, Wolfgang has encountered obstacles that have caused him to rethink his career focus. Wolfgang's first attempt at college did not go well. He describes discrimination by peers and accessibility issues with the disability office at his first university. As a result, he transferred to a larger university that had a better reputation for providing accessible materials to 
students with disabilities. In the end, however, Wolfgang changed his career from pursuing a law degree to becoming a school psychologist because he had continued problems with getting appropriate accommodations for taking the qualifying test to get into law school. He hired lawyers through his second university to fight for his accommodations for the test but eventually gave up because he was tired from fighting for what he needed as a person with a visual impairment. He was eventually able to sit for the exam; however, he did not expect to take the test and did not study sufficiently. Frustrated by the experience, he took a critical look at the career he had intended and concluded it was not for him. He says he does not feel as though judges and lawyers are able to do their jobs as they should because the system is not built to support them. The determining factor for changing his career path was his belief that if he had to work as hard as he did to sit for the entrance exam, he would likely encounter similar struggles throughout law school. Despite this hardship, he plans to return to graduate school to pursue school psychology. He believes school psychology is a better fit for his personality as he is a self-described "good listener" and "good at distracting kids."

\section{Societal Perception of Humanity of People with Visual Impairments}

All the educational intervention and mobility training that we can offer people with LV/B is ultimately limited by the fact that humanness does not guarantee equity (Kim, 2015). In my own study, this is particularly evidenced by three participants' experiences. In the first case, a young man with low vision, Nolan, recounts how he experienced overt discrimination based on his visual ability. Nolan is a college student who maintains a part-time job and is a proficient independent traveler. Although Nolan has the skills to be accepted to a public university, travel independently using various forms of transportation in an unfamiliar city, obtain and maintain gainful employment, he is nevertheless perceived as "less than" by his job supervisor, an able- 
bodied man in a position to exercise power and control over him. Nolan was told by this supervisor "he would be a manager by now if he was able to see the [food order] screen." This is a determination based purely on visual capacity, something Kim (2015) theorizes society must move away from in order to avoid severely limiting people with disabilities and marking them as othered. Additionally, Kim maintains basing perceptions of one's humanity, or lack of it, on "missing" physical and mental capacities perpetuates discrimination and oppression of people with disabilities. While other workers perceived to be "able-bodied" are promoted based, in part, on their ability to "see the screen," Nolan is instead placed in a dead-end skill pathway which he may never leave due to the way his supervisor perceives his abilities based on his visual impairment. Nolan has learned and demonstrates at his workplace skills which allow him to accommodate in a variety of situations, including viewing information at a distance. While his vision may not be his strength, he has successfully learned to utilize other senses to complete tasks successfully. Upon graduating from high school, Nolan felt prepared to pursue a career and obtain a job in large part due to the education he received. For example, Nolan notes that, Courses at [the residential school] helped me transition more than anything, I suppose. I came into that school knowing nothing and losing my vision, and going through that wasn't easy, and the school picked you up, and then showed me how to use a cane if I needed it, they taught me braille, which I used when I was first going into the dorms so I wouldn't completely look like a fool. I'd just walk by and quickly read the numbers on the dorm rooms. They taught me how to cook and they taught me laundry. ( $\mathrm{N}$ int 1 , 4/13/17, lines 125-138)

Although during our conversations Nolan never explicitly mentions perceptions of his own abilities, his description of his educational experience demonstrates he has the self- 
determination to know what he wants for his life and he possesses the positive self-efficacy to reach his goals, all of which were developed in a supportive home and school environment. Through the promotion of resiliency by Nolan's family, teachers, and community of learners with visual impairments, while he might be able to dismiss the negative attitudes and treatment of others, others' attitudes hold him back from advancement in a way that challenges his humanness (Kim, 2015).

As a job supervisor, a person with these attitudes about the humanity of people with disabilities put in a position of power and authority could have a significantly negative effect on an individual with low vision's feelings about his or her own abilities and identity, regardless of the supportive school and home environments where he or she developed those skills. A person's self-efficacy and resilience are linked to identity. When an able-bodied person devalues those skills and adaptations which a person with a disability takes pride in and has spent a lifetime developing and refining, it calls into question his perception of his own humanity and feelings of self-worth.

In the second example, Irene, a female college graduate with low vision, experienced inequitable treatment masked as "constructive feedback" during a meeting with some of her college teachers based on some of the limiting effects of her visual impairment. Similarly to Nolan, Irene demonstrated the skills to perform, gain acceptance, and excel on campus in college courses and with independent travel, she, too, was told that skills based on her ability (her inability) to see would prevent her from entering and excelling as a stage director. I learned of Irene's experiences with discrimination indirectly by watching a play she wrote and directed about living with a specific form of visual impairment. She interviewed people with the same diagnosed visual impairment from all over the world to paint a picture of the variety of lived 
experiences of adults with her particular eye condition. In one scene of the play, Irene, portrayed by an actress, was in the middle of her second-year review at her university. The instructors' main critiques were her "interpersonal skills" and ability to "read the room," however, they did not specifically indicate how she could remedy these issues. Instead, they informed her that there was "no checklist of skills" for her to acquire. She was told during a private meeting with a professor after her second-year review that,

Specifically, with regards to "interpersonal skills" and "reading the room," your main area of weakness was that you do not respond at all to non-verbal cues, and that is essential to the line of work you want to go into. But it will be okay because you are really smart and work really hard, so of course you could learn those crucial skills quickly. (I's play script, 9/2/2017, p. 22)

Interpersonal skills, reading the room, and paying attention to nonverbal cues are things individuals learn to recognize through the visual channel. In Irene's case, she was not given direct instructions on how to improve, just that she had a significant problem which would prevent her success, that there was "no checklist" for qualities of success or how to go about remedying her perceived shortcomings, and she would need to figure out how to accommodate for this perceived deficiency on her own. As an experienced TVI and COMS, I posit it likely Irene received instruction in social interaction skills as it is one of the nine curricular areas that guides instruction of youth with LV/B (Council for Exceptional Children, 2009). The reason curriculum focuses on social interaction is because nonverbal cues and body language sighted people learn by observing, people with visual impairments need to be specifically taught because their reduced vision makes it difficult for them to observe the minutia of such visual social cues as a nose crinkle or a furrowed brow. Instead, they are taught to listen to speech patterns and 
intonation to gain the same information. As a budding stage director, "reading the room" is made more difficult when your perception of detail does not extend much farther than a few feet from your body. Irene expressed she walked away feeling rejected as a result of the indirect focus placed on her visual impairment and questioned whether she needed to transfer universities because it felt as though there was something unfair about the university as she had previously received glowing evaluations.

In the third example, Wolfgang, a male college graduate with significant vision loss, describes repeated difficulty with receiving appropriate accommodations. In his most frustrating story, he recounts his experience with discrimination labeled as an "unfair advantage" when he requested accommodations to take the Law School Admission Test (LSAT).

I had a lot of issues with [my first university's] disability department. Because they kept messing up my assignments, with the enlarging and everything. Then I got an iPad, also, so it would be easier, but they weren't able to get the books from the publisher. They would still make it bigger themselves, so I would be missing sections, or it would be upside down. And even my exams were like that. So I kind of fought it out for three years and I wasn't really sure what I would do, but then I came back [to a university with a reputable disability office]. ... Then I decided to go [to school] for sociology. Because I wanted to go for law school, but then I kind of got shot down because of the accommodation issues. They wouldn't let me take the law exam with accommodationslike, the LSAT people — and they wouldn't even give me my money back. So they wanted me just to withdraw from it. (W int 1, 5/25/17, lines 166-175 \& 207-213) By refusing to provide reasonable accommodation for a person with a history of receiving accommodation for a medically diagnosed visual impairment, test administrators communicate 
only "able-bodied" individuals may gain entree, telegraphing to Wolfgang his unfitness to access the exam (and therefore law school), and effectively enacting the corporation's definition of "able-bodied" as exclusionary criterion. His exclusion from the LSAT proved a major setback to Wolfgang's self-efficacy, causing him to apply new, constricting limits to his self-image and resulting in a corresponding shift in his identity; thereafter he purposefully chose a career that did not require him to challenge or "rise above" the LSAT corporation's discrimination, ending his dream of becoming a lawyer even though his physical and intellectual abilities in no way precluded entree into that profession. Prior to the LSAT experience, Wolfgang began college believing he could achieve any goal he set. When encountering continuous (and legally questionable) roadblocks, he fought against those until he hit what he found to be an insurmountable challenge, thereafter succumbing to the debilitating effects of discrimination on self-efficacy and identity.

When, at his first university, Wolfgang did not receive appropriate accommodation, he was able to change his environment (e.g. transfer to a different university), but when he did not receive appropriate accommodation for the LSAT (at the university with a good reputation of providing accommodation for people with disabilities), he felt forced to abandon his entire life plan, even though the circumstances which led him to give up were rooted in an organization's unjust, unreasonable, and likely illegal unwillingness to accommodate for an individual's disability. The LSAT's imposition of unreasonable limitation caused Wolfgang radically to alter his self-image and then to limit himself in ways he had not previously, forcing him to "downgrade" his perceived abilities and to impose unjust limits on the expansive ways his identity and self-efficacy had been constructed in the past. The potential dismantling and degrading of an individual's identity are among the needlessly crippling effects of 
discrimination. Such discrimination and its effects cause individuals to respond in ways that artificially limit career, identity, and sense of self-efficacy and such losses are far from trivial. When an individual with a disability is forced to give up such hard-won and well-deserved ground, such a loss of self-efficacy can be very difficult or even impossible to reclaim. As a result of ongoing litigation and the intense struggle Wolfgang endured just to take an accessible version of the LSAT, he decided no longer to pursue a law degree. Instead, he began to consider becoming a school psychologist.

I was able to get my accommodations, finally. I was able to take the test and I was only like two or three points down, but, even then, like, I hadn't really studied the best I could, because I didn't think I would get my accommodations. ... So, it was kind of frustrating. So, I might take it again, or I might just go for psychology, like, to be a school psychologist. ... The thing that kind of really upset me is, like, a lot of times people, like, even judges and lawyers, don't really do their job as they should. So, like, sometimes, if you're a lawyer, you can't really do your best to stand up for something if the system isn't fully supporting it. So that's what kind of just got me upset, and even just like the trouble I had to go through just to take an admissions test. It was just...I didn't really feel comfortable going through studying for two or three years, to work so hard.

(W int 1, 5/25/17, lines 244-250 \& 259-268)

So, rather than pursuing a law degree with the intent of practicing law that would enact social justice for marginalized populations, he altered his career goals to accept the path of least resistance and shift to an outcome he could control. Much greater than a cost of time or money for a person with a visual impairment, life-altering discriminatory events then result in difficulty transitioning to one's next life stage. Professionals working with people with visual impairments 
promote problem-solving and perseverance in the face of obstacles, but what happens when an individual is rejected based on a quality that comprises a large part of his identity? What may result is a slide into self-defeating and self-deprecating mentalities that lead to a belief in unemployability, which supports the ableist perspective that people with disabilities lack capacity and agency. These destructive beliefs are further solidified by dismal employment rates for many individuals with disabilities despite strong ability and despite earnest attempts to obtain gainful employment.

The results of Nolan, Irene, and Wolfgang being told, both directly and indirectly, they could not or were not allowed to succeed in their endeavors simply because of their inability to see, regardless of the many skills they possessed that allowed them to succeed to this point, is a way in which able-bodied people remind those with visual impairments that they are not equal or as valuable as their sighted peers. Additionally, they are being judged and named as less-than and as profoundly lacking in the qualities that supposedly make able-bodied people "human" (Kim, 2015). This judgment based on capacity has the potential seriously to affect people's identity, cause them to question their self-efficacy, and result in potentially negative transition outcomes and life chances, particularly with employment. While Nolan and Irene continued with their respective life plans despite discrimination, Wolfgang was forced to significantly alter his career path and identity because he was severely limited by an entity's unwillingness to provide appropriate accommodations.

TVIs and COMSs tell people with visual impairments "you can do what you desire given the appropriate accommodations" and teach them problem-solving so when they are told "you cannot do this because of your visual impairment," they persevere to overcome challenges and road blocks. The issue, however, is that this persistence over time can wane when a person is 
continually told she lacks the capacity based on something she is unable to control (and for which she is trained to accommodate), leading to a kind of ability-driven battle fatigue (Kim, 2015). She may begin to internalize this belief, dismantling the positive identity she, her teachers, and her family have worked tirelessly to construct. When a person's humanity is questioned because of a perceived lack of skills a radical restructuring of societal perceptions of disability could draw from Kim's notion of unbecoming human to challenge and shift perceptions of disability to a strengths-based perspective.

Unbecoming human, if adopted as a theory of intervention, is a means by which society can eliminate the value placed on capacity, agency, and ability and in so doing, move away from a perspective that oppresses and discriminates against people with visual impairments. If members of society think of themselves as objects and distance themselves from a preconceived checklist of what it is to be human, then they can include people who historically have been excluded because they did not meet invented, ableist criteria required to be considered "human" and can promote people regardless of their ability to "see the screen," "read the room," or require accommodations to take an entrance exam. In the following claim, I draw from my data to address how experiencing vision loss in an individualistic society affects a person's perception of her humanity and these implications in the (re)construction of her identity.

\section{Societal Perception of Humanity and Identity Construction}

Youth and young adults with visual impairments who experience degradation of visual ability endure intense fear, depression, and reduced confidence over their vision loss because they understand the societal value placed on the ability to see and how this change may challenge their status as "human" (Kim, 2015) because of the shift in the ways they engage with the world and how others perceive them. With any major life change comes an adjustment period. When a 
person goes from having typical vision to having low vision, they have to modify not just how they interact with their environment but also how they perceive themselves and their (dis)ability. A diagnosis of a visual impairment presents its own unique and mechanical challenges; these issues are enhanced when a person is harassed by peers and lacks teacher and administrative support to obtain necessary accommodations to access information taught in class. These experiences often lead to feelings of inadequacy rooted in the treatment by others as less-than because the individual is not excelling academically in the way he was once able and is acutely aware when he is not supported by peers and staff during this delicate transition. This phenomenon emerged in the words of several participants. Amelia, Maverick, and Nolan all express similar feelings of fear, depression, and loss of confidence as their vision declined during differing life stages. Amelia shares her fears for her future when describing her recent bout of vision loss:

I think that something that's kind of scary is that my vision in certain areas has been deteriorating and nobody can figure out why. When they do the tests on my eye, they don't see any changes and we don't really understand what's going on with that and it's honestly very scary because I went from having a normal person's night vision to having absolutely no night vision and then I went from, you know, my eyes being perfectly clear to suddenly having blurs and stuff in my vision and nobody knows what's up with either of those things. What scares me about that is that my vision was never supposed to deteriorate so it's like, “Am I eventually gonna go blind over this? What is...happening here?" (Am int 1, 4/13/17, lines 303-315)

Earlier in the interview, Amelia describes experiences with bullying in her home school district because of her visual and physical impairments, evidencing how she has internalized that 
she does not belong to the world, to social groups, or organizations in the same way as her ablebodied peers. Her fears for her deteriorating vision are also rooted in living in a sight-dominated society where a person's perceived level of independence is based on her ability to see. Young adults with visual impairments feel the effects of reduced vision even more intensely because the amount of information they are able to access and others' perceptions of their intellect are directly related to the amount of vision they possess. Take, for example, when a sighted person provides directions to a point of interest. In lieu of street names and cardinal directions (e.g. North, South, East, West), people typically use visual landmarks and vague terms such as "over there" while pointing to a nondescript location. When a person with low vision who does not physically appear to have a visual impairment asks for more specific directions, public perception is that he lacks intelligence and understanding to follow the "clearly" stated instructions. In the case of vision loss, losing the familiar way in which a person views and navigates the world can spiral her into depression and shake her confidence, especially when she is aware that blindness is a fear for many because it requires the individual to engage in everyday tasks in a completely different way than that to which she was once accustomed and poses unique risks to one's safety. To compound and deepen the ableist's perceived shortcomings of young people with LV/B, able-bodied people often over-sensationalize the abilities of young adults with visual impairments as "brave" and "inspiring," yet they still underestimate their capacity as contributors to society by overhelping and treating them as though their cognition is affected, not their vision. Over-sensationalizing young people with visual impairments' learned accommodations reinforces the public perception that little can be expected of individuals with LV/B regardless of intellect, training, or ability and serves further to de-humanize those with vision impairments. This is particularly evident when people carrying long white canes attempt 
to cross the street, navigate stairs, and use public transportation. Sighted individuals will occasionally grab the person using a white cane and "help" him cross a busy street unsolicited. Another participant, Wolfgang, a graduate from a four-year university and a white cane user, notes that "... some people assume that I can't even go down the steps, so they try to push me on the elevators" (W int, 5/25/17, lines 477-479). These are just a few of the many ways in which society tells people with visual impairments that it does not believe they have the skills, physical ability, or intellectual capacity to travel independently within shared space.

Maverick, a college graduate who attended a residential PK-12 school for the visually impaired, echoes Amelia's fear as he describes the time when he lost much of his vision as a teenager. He came to view his vision loss as a significant threat to independence.

I kind of went into this, in my opinion, massive depression of "What's there to life now? What am I going to do? I don't know...How am I going to navigate things?’ Like I used to be...I would ride my bike across the town from my house to my grandmother's house. It was about two and a half miles. And so my parents wouldn't want me to do that anymore because well, I think they were afraid I was going to hurt myself. (Ma int 1, 4/26/17, lines 207-213)

Maverick's experience demonstrates his awareness of how his life would change because of his visual impairment and his fears and perceptions mirror society's. While his family supported him, they still expressed their fears (and initial doubts) for his safety as an independent traveler. These fears, if not kept in check, had the potential negatively to affect Maverick's independence. If he allowed his family's fears for his own safety to become debilitating, he would not have been willing to learn to cross the street or use public transportation, significantly altering his identity as an independent traveler. Additionally, with the lack of academic and emotional 
support from his home high school, it is unlikely Maverick would have gained the independent skills necessary successfully to transition into adulthood had he not transferred to a residential school for the visually impaired. In his home high school, he did not have access to a TVI or COMS to teach him the accommodative and independent travel skills that are vital to successful transition outcomes (Wolffe \& Kelly, 2011). This lack of resources and the public's ignorance about the ways in which people with visual impairments are taught to travel independently leads society to infantilize this population which translates into an inability effectively to convince employers of such skills, as evidenced previously by the limitations placed on Nolan by his job supervisor.

The dismissive ability-centric mentality of Nolan's job supervisor is one of many obstacles he has overcome connected to his reduction in visual acuity. He describes how acquiring his visual impairment made him more aware of others' perceptions of him and expresses his frustration with his treatment by others because of his vision loss:

Some people judge me for my visual impairment and I saw it coming in a way, because, before [the residential school for the blind], people did it then because people are just...people. Well, the first guy I met, we were in orientation [at the university] and we ended up seeing each other when we came in for actual college, like the first week or so, and he was like, "Hey let's go work out at the gym," and I'm like, "Yeah, cool." So we were over there hanging out and everything and, like, we were cool, and he asked me for time and uh....Yeah, to read the time, and we were in the pool doing laps and stuff and I'm like, "I can't read the clock up on the walls," he's like, "Why?" and I told him I had a visual impairment and then, like, two hours later we ended up not hanging out and then he didn't talk to me anymore. Yeah, so...before that he was all fine and everything. 
It's happened a couple times, but, I mean, in classrooms, people just look at you different...Cause you're looking at things up close....and...you're just different than everyone else. Everyone else expects you to be able to see the board and see the laptop from at least five feet away (leans back from table) but here you are with your face in the screen (leans forward across table) and everyone's...lookin' at ya. I don't like it, but, it's life and people are gonna judge you and you can't do anything about it. I mean, what am I gonna do? (N int 1, 4/13/17, lines 166-168, 213-224, \& 229-242)

Treatment as incapable by others has forced Nolan to go "into hiding" about his visual impairment. He chooses to share his visual needs only with those whom he has developed a trusting relationship. Furthermore, Nolan talked of his struggle to "pass" as a sighted person in a sighted world by not openly utilizing tools that would "out" him as visually impaired. When asked about his ability to drive, he simply tells people he does not have a driver's license, not that he is medically ineligible to drive due to his reduced visual acuity and field of vision. Learning of Nolan's experiences with his peers is disappointing and disheartening because as teachers, we try to instill the value and importance of advocation and accommodation so young adults with LV/B can live their best lives. Students are taught to carry a long white cane visually to identify themselves as having a disability and verbally to share their needs because educators believe that knowledge leads to understanding. The more a person is rejected and mistreated on the basis of perceived lack of ability the more the voices that tell the young adult with LV/B he belongs and he is capable become quieter and quieter until they disappear or are replaced by a voice that claims he lacks agency, capacity, and all of the qualities that would allow him to be an accepted member of society and fully human (Kim, 2015). In this aspect of the phenomenon of youth with LV/B navigating their world I see disturbing parallels to the everyday, cumulative, 
crushing harm of racial microaggressions, in particular the racial microaggression that occurs when a teacher asks the one person of color in the room to speak for all people of color and to "educate" white people in matters of race. The able-bodied world expects people with disabilities to educate them and to speak, in many instances, for all people with visual impairments.

Nolan also describes his vision loss as a teenager as a confidence crusher because it came during a pivotal time in his life: "I guess, more than anything, losing my vision just shot confidence right out the window. So any confidence I had that I was getting for being able to drive just went out the window when I lost vision" ( $\mathrm{N}$ int 1, 4/13/17, lines 305-308). Nolan's loss of confidence points to a common theme that emerges from many participants' data: driving and nondriving play a key role in identity construction and confidence building for youth and young adults with visual impairments because driving offers a level of independence, freedom, and responsibility that one does not gain through any other means and because U.S. youth in particular are socialized toward driving a vehicle and equating that with an important step in independence and transition to adulthood. For example, the opportunity to "get up and go" anywhere at any time is realized when an adolescent has a driver's license and access to a car. His world becomes much larger because he is not limited by time or distance when he is able to drive; he is deemed "responsible" for himself and others. His options for employment are far reaching and the responsibilities placed on him by his family increase because he is mobilized by the ability to drive. This is vastly different for youth and adults with visual impairments. Instead, the person with LV/B relies heavily on family, friends, and available public transportation to arrive to points of interest, especially when they are a reasonable distance from the individual's home; this is often perceived by all parties as a form of dependence and a denial of the freedoms young people expect as they transition to adulthood. Additionally, job and travel opportunities 
for youth and young adults who are visually impaired are limited by the transportation options in their area. While their sighted peers are gaining responsibilities, building their confidence, and increasing independence due to their ability to drive a car, adolescents with visual impairments are not afforded the same opportunity for growth in the same ways as their peers. They may feel, and are often treated, like outsiders when they are unable to earn a driver's license because they are required to learn a different, nontraditional way to gain independence.

Although people with visual impairments are able to gain independence through alternate means, the fact that it is not the same form of independent travel (e.g. driving a car) makes it difficult for able-bodied people to understand and appreciate the complex nature of travel for young adults with visual impairments or to credit them with being highly skilled and competent young adults. The perception, rather, is that the person with a visual impairment is incapable of independent travel because she is unable to operate a motorized vehicle when the reality is that it requires extensive training and development of a wide array of skills to use public transportation effectively. Irene recounts an exchange she had in college pertaining to the difference in the way she traveled in comparison to her friend:

I had a college friend who had never taken public transit in his life because he was from a very far-north suburb. Got a car when he was 16, he used that car in high school and college and beyond and to him, he never needed to use public transit so when I would describe how long it would take to get to certain places or where I was at in a route, saying I'm on a particular train line, he would have no clue sometimes. (I int, 5/19/17, lines 1149-1154)

While her friend did not express doubt in relation to her skills as an independent traveler, he could not appreciate the time, planning, and skill involved in taking multiple subway trains to 
arrive to a destination because he was in a situation that did not afford him the opportunity or necessity to use public transportation. Due to his geographic location, Irene's friend was limited by his transportation choices. As someone moves away from major metropolitan areas, there are fewer options for travel outside of driving a car. This is in large part why many people with LV/B opt to live in or near larger cities so they can maximize their mobility. Irene's mother, Annie, purposely moved her family to a place that had multiple forms of reliable public transportation because she and her husband knew that access to mass transit meant access for her daughter with a visual impairment. This point is further evidenced by Amelia's professed need to live in a place with reliable mass transportation.

I guess the only other thing is like where, what kind of place am I going to live when I get out on my own because I have to be somewhere where there's mass transit...because so many jobs and so many places rely on driving, nowadays, to get places. (Am int 1, $4 / 13 / 17$, lines 351-356)

Irene's and Amelia's experiences demonstrate how having a visual impairment not only changes the way society perceives a person with a visual impairment, it also requires the individual to consider accessible transit when creating future plans to allow one to be as independent a traveler as possible. This data selection also exposes the value of access to reliable public transportation and how this enhances a person's independence. Irene's college friend could not fathom the length of time it took for her to use the train/public transit because he was able to drive in a car, greatly reducing his travel time and interdependence on many variables: the transit schedule, disruptions, etc. Public transportation can be empowering, but also limiting, depending on the reliability and availability, which is why many people with LV/B who do not 
drive elect to live in larger metropolitan areas to increase their independence by accessing these services.

It is difficult for people to imagine life after vision loss because so much focus is placed on the visual. As society reminds people with visual impairments of the areas in which they are "lacking" (e.g. ability to drive, participate in spontaneous social interactions; Sacks \& Rosenblum, 2006), educators can promote the creation of a positive identity and assist youth and young adults with LV/B to regain their confidence. Maverick explains,

When I came to [the residential school] and learned what I needed to learn, I gained all my confidence back. I'm still scared or afraid to go to new places by myself, but I always think to myself, "You're never lost. You have a smart phone." But if that were to not work there's people that you can go up to and ask. Yeah, so that's how I think of things now. Is that you're never lost in this world. This world is not that large because there's seven million people in this world and one or two people should know where you're at. But before that, I was always scared of, well "I'm going to get lost. I'm going to freak out and stress out." So that's how I was for most of the time that I was visually impaired, and so I gained my confidence back when I learned mobility and orientation. (Ma int 1, 4/26/17, lines 214-224)

Without support from family members and teachers, knowledge of available resources, and internalizing an identity as an independent traveler, it is reasonable to surmise that these young adults with visual impairments would only aspire to reach the low expectations of the able-bodied society in which they live. This is especially likely when they have been treated as lacking in the ways that make people human (Kim, 2015). Law says pedestrians are to be protected above all traffic and there are particular laws for the blind (e.g. white cane laws), our 
culture nevertheless privileges the freedom of driving and transit far above the frailty of the human body. When referring to a college friend of hers who had never taken public transportation because it was not available in his geographic area, which presented a gap in his understanding of the time and planning required to use public transportation, Irene notes,

I thought that was just so interesting that there are people who have enough privilege to not need to understand public transportation. I don't mean privileged as in class privilege, or wealth, or anything but just the ability to drive coupled with owning a vehicle. How that makes you exempt from needing to know about public transportation at all. (I int, 5/19/17, lines 1155-1159)

Additionally, all participants note that little to no focus was placed on pedestrian travel or laws protecting individuals with visual impairments during driver's education courses. The privileging of driving as a part of transition to adulthood, independence, and competence is given a false prominence by society. The knowledge, skills, self-efficacy, and boldness young adults with visual impairments must acquire and practice demonstrates far more by way of the hallmarks of independence, planning, responsibility, logic, and knowledge acquisition than does simply driving a car, yet such young adults are still regarded as less able — and therefore, lessthan-human (Kim, 2015).

\section{Societal Perception of Humanity and Driver's Education}

When schools require students with visual impairments to participate in driver's education when they are medically ineligible to drive, they devalue the ability of the person by reinforcing the visually impaired are never going to be part of this aspect of society, thus perpetuating ableism when schools should be giving young people with LV/B appropriate instruction to aid in transition, including real-life skills and how to become resilient young 
adults. Participation in driver's education satisfies a graduation requirement in some school districts. By adopting and executing a blanket policy that obviously caters to certain populations and ignores others, school boards and administrators are neither making decisions based on the entire population of students they serve (including those with disabilities) nor considering the social-emotional implications of taking the classroom portion of driver's education for students with visual impairments. This oversight speaks volumes about the values of school leadership in terms of students with disabilities by ignoring the unique needs of all students attending their schools. It also demonstrates the lack of insight policymakers have regarding the effects of their policies on specific populations. Tommy, a recent high school graduate enrolled in community college courses, laments about the hit his self-confidence took while in driver's education, It didn't really help it, because like I know I'm not going to get to drive. Why just punish me even more by [making me sit in this class and] giving all these other kids their permits at the end of the semester [when I don't get one]? (T int, 6/1/17, lines 755-757) Amelia also recalls her distressing experience in driver's education, as it was also a graduation requirement in her rural town.

It was really uncomfortable. It's like, you're surrounded by all these people that are learning how to drive, and it's like, “Why am I here?" It's really uncomfortable, it's like, most of the time I don't really care that I can't drive, but that one class, just really front and center, made it...awkward. And it really makes you stand out from your peers when they get to the driving session and you're not, er, you're not able to do anything but watch the other people. They're like, "Why is she standing there by herself?" Cause they don't know. It just made me really uncomfortable and it made me feel, you know, even more ostracized from my peers. (Am int 1, 4/13/17, lines 374-383) 
To subject students to these feelings of inadequacy that cause them to question their self-efficacy and ability is inhumane and runs counter to the goals of educating students with disabilities: creating self-assured and self-determined members of society. It also does nothing to promote resiliency in youth or teach them "real-life" skills. Instead, it acts as a destructive force on one's self-worth and ability to transition to adulthood while at an impressionable age. Another participant, Wolfgang, did not take driver's education while in high school but still read the Rules of the Road book because he and his mother believed the information drivers learned was important for him as a pedestrian. He agrees with Tommy and Amelia that requiring participation in the classroom portion of driver's education "would kind of be discouraging for people. Especially if they can't really take the class. Like if they wouldn't qualify [to drive because of their vision], because it would just be a reminder that they can't really do it" (W int, $5 / 25 / 17$, lines $745-748$ ). It is difficult enough not to share driving as a rite of passage with their peers, it is irresponsible for leadership knowingly and forcibly to put students with visual impairments in a situation that acts as a constant reminder of how they differ from the sighted world and that they will never be deemed capable members of this group in society, effectively reifying a second-class-citizen status among students with LV/B. All told, a few participants had strong, even visceral reactions to participation in the classroom portion of driver's education while others saw value in completing the classroom-based coursework.

While none of the participants advocated for requiring driver's education coursework to earn a high school diploma, some did not experience the intense emotional response described by Tommy and Amelia. Unlike participants who grew up in rural areas, Irene's involvement in driver's education did not affect her self-confidence and self-esteem because she was aware and proficient in the use of other transportation options available to her as a resident of a large, 
metropolitan area. This was in large part due to her access to consistent orientation and mobility instruction. Tommy and Amelia lived in towns where they had access to a fixed-route bus system, however, traveling beyond the confines of the town created logistical issues and made them feel more limited and stifled as a result. In Irene's experience, she was required to take the classroom portion of driver's education but her family advocated for a "pass/fail" option to reduce the pressure placed on earning a grade that would affect her grade point average in a course that was deemed pointless by the family, as her vision disqualified her from obtaining a driving permit. Irene agreed with this decision and notes she benefited from some of the information covered because she holds the belief it is, "important to be literate, even in areas that may not appear to pertain to you."

Irene's perspective regarding "life literacy" is in line with some statements made by Maverick and Nolan, the brothers who experienced vision loss at the age they became eligible to begin learning to drive. Looking back, both maintain information gained from the course was valuable enough to warrant their participation in the classroom portion. Maverick even considers the value of "observing" the behind-the-wheel experience as a means to provide background knowledge to add depth to orientation and mobility instruction.

Because trying to think - and I've thought about this for a while when you asked me the question. If I didn't know driver's ed, or if I didn't have that base of understanding of driver's ed, I wonder how that would be different when I learned O\&M. I don't know. Because [the orientation and mobility specialist] taught me the same types of things or the understanding of things of what I knew in driver's ed, too. "So, this is what is going to happen" and so I just agreed and then I told him, "yes, I know that understanding because...." I guess that's my answer. I don't know how it would be different if I didn't 
learn O\&M, or if I didn’t know driver's ed compared to O\&M. But it did help me to build upon...so it did help me. I'm trying to phrase this. It took me to a next level of O\&M instruction because I did know about driver's ed. So, it did help [the O\&M instructor at the time] to try and build off of what I did or I did not learn in driver's ed or understanding of the [layout of the] streets. (Ma int 2, 5/16/17, lines 291-305)

At first, Maverick did not believe taking driver's education prior to losing his vision was as important as his instruction in the specific ways in which a person with visual impairments may access his physical environment through systematic instruction by a certified orientation and mobility specialist. However, upon further reflection, Maverick questions whether his depth of knowledge in independent travel would have been as comprehensive had it not been for his background knowledge of the laws governing motorized travel in the built environment. Maverick's viewpoint is important because his experience further supports the individualization of curricula for young people with visual impairments.

Nolan echoes his brother's belief that driver's education may be beneficial for some because it provides the pedestrian/passenger the opportunity to learn about how drivers are taught to behave in certain situations so the visually impaired may make informed decisions when a driver is engaging in unsafe behavior. He also notes that not being able to drive is a sensitive topic for him to this day.

Taking driver's ed, it's um, an experience, in a way. I mean, even though I didn't take it for long, it gave me an idea, um, it will help you understand what drivers are...thinking...even though you cannot.... I don't know, it'll kind of put you in their seat, but you'll never be in their seat. You just know what they're...processing...in a way.... I mean, I don't think driver's ed impacts visually impaired kids as one could 
think. Um, we never drive; we want to, but we're never going to.... Um...just knowing what a driver's going to do...but you can never predict what a driver is gonna do, either, because some people are just reckless, and you still have to rely on yourself. You know the basics and how things should go but you'll never know exactly how they'll go. ( $\mathrm{N}$ int 1, 4/13/17, lines 373-378 \& 386-394)

For my participants, the underlying issue is not whether driver's education is made available in public schools, but that it is required for graduation in some districts, which serves as a reminder of one arena in which people with visual impairments are excluded. Each participant named at least one concept taught in driver's education that was valuable to him/her as a traveler, however, this value did not outweigh the negative social and emotional experiences for some participants. The variance in participant experiences, geographic regions, and age of onset of vision loss makes it impossible and ill-advised to advocate for blanket policies regarding student participation in driver's education, especially when those students include adolescents with disabilities. In the following section, I explore ways in which societal perceptions of the capacity of those unable to drive compounds the social and emotional effects of participating in driver's education for nondrivers and the effects of this perception on identity construction.

\section{Societal Perception of Humanity Based on Nondriving}

The individualistic nature of American society makes it difficult for people with visual impairments to reconcile their need for assistance with their desire to be independent, which is based in part on the idea that ability equals humanity (Kim, 2015). American society differs from much of the rest of the world in that success is measured by the actions and abilities of the individual, not of the collective community and is largely focused on humans demonstrating autonomy. Teenagers are socialized to take pride in exhibiting independence and gaining 
responsibility as evidenced by their ability to drive when they turn 16 . Transition to adulthood takes the form of getting a full-time job (or attending college), moving away from home, and paying for one's own expenses. When a person is unable to exercise the same level of independence as her able-bodied peers existing in an individualistic environment, her ability to contribute in the same way is questioned. Depending on others for everyday tasks can reduce a person with LV/B's notion of autonomy because she is unable to execute said tasks without the assistance of another person. Providing for oneself is highly valued in the U.S. and when one is unable to do so, it threatens his identity as a truly independent person. Some of the ways in which people with visual impairments take back control of their autonomy is by making the decision to refuse rides or offer reciprocal services as reimbursement for services rendered. These feelings come to light when participants express their desire to be as independent as possible by declining offered rides. Some participants prefer reciprocity if they do accept rides because they want to demonstrate their ability to provide for those who provide a service for them. They also do not want to be perceived as a "burden." One participant notes he chooses to walk to his destination in lieu of accepting a ride, even if that walk is two miles in inclement weather. Another participant, Devon, a college student in his late 20s, talks about his experience with asking others for rides:

It put a burden on me to ask people for rides home [when in high school]. At that time, me and my sister didn't get along really well, so she would never give me a ride, even though we lived at the same place. My friends hated her for that, which is cool. I don't know if it affected my confidence or whatnot, but I'm kind of stubborn in some ways. I don't like asking for rides or help, so I try to figure it out on my own. I have a pretty good 
support group of friends who were always willing to give me rides. (De int, 6/2/17, lines 261-268)

Devon refers to a "support group of friends" who provided him with transportation when in high school and from whom he felt comfortable accepting rides. This form of support is invaluable because it reinforces to the individual with a visual impairment that needing and asking for assistance are acceptable, even if he is surrounded by messages that say they are not, allowing the person with a visual impairment to feel in control. Shifting to a societal emphasis on collectivism and/or unbecoming human (Kim, 2015) affects participants and students with visual impairments by removing the value placed on the capacity of the individual. This is important because having a visual impairment results in an increased reliance on others to obtain visual information and to engage in some tasks (e.g. travel in rural areas). By measuring success of the collective community and the ways in which they support those in their community instead of focusing on the lack of capacity of one person allows fostering more inclusive environments that do not reject or exclude based on ability.

A common concern for participants with visual impairments is thinking of themselves as a "burden" if they must ask someone for help. Such a frame exemplifies the oppressed adopting the perspective of the oppressor (Freire, 2015) and there is something inherently unkind with and uncharitable about such a characterization. People with visual impairments are influenced by and urged to serve the individualistic mentality because they have lived for years operating in a system where certain groups are, in essence, set up to fail because they can only thrive in a collectivist enterprise. U.S. society centers individualism because of the way our infrastructure and information gathering have been designed to benefit the sighted and requires each person to fend for himself and compete against others for finite resources, positioning those who cannot do 
so without support lower in the social hierarchy or even rendering them less-than-human (Kim, 2015). This is evidenced by participants' feelings of "being a burden," and thus experiencing a reduced sense of autonomy because they are required to rely on others for assistance. My experience with some adults with visual impairments is that they want to be as independent as possible and some feel very strongly about wanting to get places without help; even if that means taking a bus for two hours to get across town. They do not want to be seen as a "charity case" and feel strongly about making sure the person offering a ride is not inconvenienced by the offer. The issue becomes more complex because public perception is that people with visual impairments are underestimated in terms of capacity because we live in a society that is highly visual and individualistic. I regularly encounter folks who find it difficult to imagine a person who is blind traveling independently using public or para transit. Because most folks live outside urban public transit hubs they require use of a car conveniently to reach a destination and largely have little experience with public transportation. I am also frequently told when working with people with LV/B by able-bodied adults that, "I could not imagine losing my vision. The hardest thing would be relinquishing my driver's license. How would I get around?” This focus on rugged individualism is so engrained in the U.S. psyche that a few of the participants in this study who experienced reduced vision asked themselves the same question: "How will I ever get around if I'm blind?" Imagining an alternative to the highly valued driver's license and access to a car can be difficult to wrap one's head around. Not only that, it can be emotionally devastating when one realizes she is unable to exercise the level of independence she envisioned for herself because she is reminded by others of "how difficult it must be," "how long it takes" to reach a destination, and the ways in which she is perceived as less-than autonomous based on visual ability. Participants with visual impairments have shared frustrations regarding the length of time 
it takes to use public transportation and that they "wish they could drive," which I believe, in part, is based on limited availability to public transportation in certain areas, but I believe is largely based on society telling them that independence is valued and interdependence is not. In the next section, I describe the ways in which young adults with visual impairments overcome societal perception of their humanity to construct healthy identities and live fulfilling lives.

\section{Overcoming Societal Perception of Humanity to Construct Healthy Identities}

Knowing they exist in an ableist society, some people with visual impairments feel it is necessary to go the extra mile to prove they are as capable, and as "human," as their able-bodied counterparts. No amount of mobility training, technology instruction, or helping with selfefficacy can surmount the societal oppression facing people with visual impairments. The population of people with LV/B cannot simply confront this oppression by being twice as competent. Such a tactic is likely to work about as effectively as it has worked for people experiencing racial discrimination. We, as a discipline, look at the individual and we differentiate our instruction based on individual needs. The type of problems my participants are relating are showing, in stark contrast, that we as a discipline need to look at this issue at a societal level in different ways than we have in the past. I see Kim's (2015) theory of unbecoming human as a way in which we can embark on this process. The lengths to which people with visual impairments go to prove they are as capable as able-bodied folks exposes the existence of a different standard for "humanity" and also presents a "no-win" situation because in our culture people with a disability are oftentimes seen as less-than-human. Maverick expresses this need for demonstrating capacity above and beyond his peers:

Currently I'm studying for my undergrad in business and marketing and then my masters will be in communication.... Well, I'd like to keep it broad.... It's more or less a buffer 
for me because I have a disability to show that I had my master's degree and I've proven myself that I can do well and I know the knowledge well enough [or] better than somebody with a bachelor's degree. (Ma int 1, 4/26/17, lines 66-72) Extending their education to make up for something that is perceived to be physically "missing" or because they are viewed as incapable based on something they cannot control is not a concern for most folks. Instead, able-bodied people further their education to make themselves more marketable, to stand out from their competition. In a society that believes it has made great strides in enacting equal rights and access for all, this is a concern for a 25 -year-old college graduate with low vision in 2017 because he has experienced discrimination based on his visual impairment in his educational and professional careers.

Maverick understands discrimination is a part of his reality as a person with low vision and plans to further his education to prove he has more to offer despite having a disability, because he sees he must present to a potential employer as "more than" a traditionally sighted person. Instead of focusing on the ways in which all deprecated communities are perceived as lacking, educators and researchers entering these communities must highlight the desires, skills, and successes, not just the obstacles individuals have overcome or ways in which they are disadvantaged. Focusing on a strengths-based perspective has the potential to change hearts and minds because people can relate to an individual's desires for equity and independence. Once a story is told and the listener or reader connects to that person's experience, stories often become unforgettable. By focusing on individuals' desires instead of that individual's capacity (or incapacity) for certain tasks, society can move away from the value (and the very definition) placed on ability and agency. This shift creates a means by which society can move toward Kim's (2015) theory of unbecoming human in order to avoid severely limiting people with 
disabilities and marking them as other and profoundly lacking in autonomy and agency. Internalization as "damaged" deeply affects self-efficacy, identity, and a person with a disability's transition to adulthood (Tuck, 2009). "Damaged" individuals logically develop and experience low self-worth that can persist over one's lifetime and are more likely to be dragged down by obstacles that get in the way of attaining one's goals. Additionally, identifying as "broken" may influence an individual's desire ever to set goals because of profound doubt of one's ability to achieve goals. Attributing failure to brokenness results in a self-fulfilling prophecy of incapacity based on internal factors and perpetuates the persistent cycle of unemployment, underemployment, and other difficulties for individuals with LV/B transitioning to adulthood, further cementing individual and community identities as "lacking."

While participants have experienced a positive transition from school to adulthood due, in large part, to their resilience, familial support and skills acquired during educational training many indicate a partial internalization of being "disadvantaged" when discussing the desire to pass as "sighted" and, thus, overcompensate to make up for "missing" parts. Nolan and Maverick's mother, Mickey, relates her perception of what her boys experience when engaging with the public.

Instead of explaining that [they have a visual impairment and what their needs are], I don't think they want to divulge that [all the time]. I really don't. Because they don't want... I know for a fact neither one of them want pity. (Mi int, 5/2/17, lines 993-995) Identifying as "broken" or "damaged" presents a risk for individuals because their choices are reduced either to overcoming adversity or succumbing to the label of "disadvantaged." Surprisingly, each of my study participants has persevered in a different way, likely because resilience was taught and promoted by families and teachers, which, had this not been the case, 
they would have likely experienced poor transition outcomes. In hearing about Maverick's, Nolan's, and Irene's experiences with discrimination, it is unjustly stigmatizing that they feel the need to overcompensate this much to be perceived as capable by able-bodied folks in positions to exercise power over them, which, even after this colossal effort, is not a guarantee. This desire to overcompensate to make up for "missing" abilities is important for educators because they need to know that educating the individual is not enough; we must begin working on a societal level to awaken modern society to the ways in which people with VI (and other disabilities) are discriminated against so we can work to alter the perceptions of incapacity and autonomy so deeply engrained in the U.S. subconscious.

This feeling of "having something to prove" is also manifested in the belief some participants with visual impairments hold that it is necessary to be ultra-literate in transportation and driving to prove they are as capable, and as "adult" as their able-bodied counterparts, despite being unable to drive. Although Maverick had typical vision when he took the classroom portion of driver's education at his high school, he maintains that it would have been valuable for him to participate in the course as a person with low vision as well.

If I didn't know [the information taught in] driver's ed today, I would be asking [the driver] all these questions. "Why do you have to do that?" or "why did you have to do that?” And then it just sounds...it just makes me look stupid, I think. (Ma int 2, 5/16/17, lines 375-378)

While the inability to participate in the same rite of passage as his peers and thus achieve this aspect of transition to "adulthood" was emotionally devastating to Maverick, ultimately his biggest concern is not how he will become mobile, but that he will appear "stupid" to others by not knowing what drivers know. He incorporates this need to know specific information into his 
identity and worries how others view him. Maverick's understanding of "smartness" is having knowledge, or cultural capital, that is valued by society. In this case, looking, behaving, and thinking as a sighted person and knowledge of driving rules and the built environment are highly valued. If he does not have the knowledge, then he is not perceived as "smart", and by default, "looks stupid." Hatt (2012) describes "smartness" in her one-year ethnographic study of a kindergarten classroom as a cultural construct used as a tool of control and social positioning, not ability based in biological capacity. "Smartness" is a "cultural practice we use to put meaning in ourselves and others" (Hatt, 2012, p. 457). Smartness is also based on peoples' abilities to meet society's social and behavioral expectations (implicit theory of intelligence), not data (explicit knowledge). Maverick's motivation to maintain the social positioning of a "smart," capable person is what keeps him learning more than his able-bodied peers. One could argue that Maverick's overcompensation demonstrates a dissatisfaction with himself, his abilities, and poor self-efficacy. If this were true, then Maverick would likely have much difficulty transitioning to life after high school and, more recently, the working world. Instead, I maintain Maverick's dedication to learning as much, or more than, his able-bodied peers shows he well understands societal norms and has the wherewithal to arm himself for future obstacles with the tools necessary to overcome them as a resilient young adult.

Some young adults with visual impairments believe that demonstrating capacity by learning more than their able-bodied peers is important and note that as pedestrians, they are also expected and held accountable for knowing what drivers know.

Then, it's also like people who don't drive are still held accountable for knowing at least how to be a good pedestrian or knowing, being accountable for knowing what cars, what 
people who drive have to go through. I don't mind being accountable for knowing that because I think it's good to just be literate. (I int, 5/19/17, lines 1159-1164)

The idea of being responsible for information that may or may not be relevant to a person who is unable to drive exposes the internalization of society's message to people with low vision: not knowing how to do something, regardless of its relevance to your situation, makes one look unintelligent and inferior to able-bodied people and makes people with disabilities stand apart from those deemed able and therefore fully human. Recognizing and understanding a person's limitations and compensating for those limitations is a way in which people can overcome certain challenges to realize their goals. The issue here, however, is that instead of simply compensating for one or two perceived weaknesses (e.g. poor organizational skills or time management skills), young adults with visual impairments attempt to soak in information not just to compensate for the effects of vision loss, but also to combat public perception of incapacity and discrimination because they are repeatedly treated as less-than. Constant focus on the ways in which a person is "damaged" or "lacking" chips away at and threatens to obliterate a young person's self-efficacy, self-worth, and identity as an independent, valued contributor to society. Often, young adults with visual impairments recognize discrimination based on visual ability is inevitable and, as a result, arm themselves with knowledge so they may problem-solve effectively and surround themselves with a support network of people who see them for the qualities and skills they possess, not for what society at large thinks they lack. Maverick, for example, has built confidence in travel and accessing information using his other senses by receiving disability-specific education in areas of the expanded core curriculum, in spite of what could be labeled his "missing" abilities. He has adopted his mother's belief he is not limited to mere survival through government-supported financial assistance, instead, he aims to thrive 
through obtaining competitive employment to support himself, his wife, and future children. Maverick continues to seek opportunities to learn and since completion of this study, has acquired a job as a civilian working with the military. He hopes to continue on his current path, learning and developing skills that will allow him to progress up the ladder in his chosen career field.

As I explain in participant vignettes at the start of this chapter, each participant has either reached or is currently striving to attain self-imposed goals. Various roadblocks have forced participants to change the means or routes in some instances, but nevertheless those individuals push forward with determination and creativity. An upshot to constantly having to overcome adversity is that participants have learned to adapt and to advocate for themselves in order to navigate educational and professional systems by learning their legal rights and how, as a person with a disability, best to meet their needs and desires. While educating oneself does not solve the societal issue of perceived incapacity, it does empower young adults with visual impairments to push back against discrimination and treatment as less-than-for themselves as well as for others. Community support and education have helped to create self-assured and self-determined individuals with low vision who have constructed healthy identities as capable, interdependent adults and who have found their place in a world dominated by sighted, ableist perspectives.

I have worked to evidence powerful claims arising from my data through the use of my theoretical frame, revealing the ways in which society perceives and treats young people with visual impairments and how such attitudes and treatment play a role in developing self-efficacy, self-determination, and identity and how these experiences can affect transition outcomes both positively and negatively. All of this knowledge was generated from a seemingly innocuous research question about the educational programming of youth and young adults with visual 
impairments. In the next section, I present my findings and the implications of my findings not only to the field of visual impairments but to society at large, as well as provide recommendations for practice and directions for future research. 


\section{CHAPTER V: FINDINGS, IMPLICATIONS, \& RECOMMENDATIONS}

I began this journey with the goal of creating a list of concepts that can be taught to adolescents with visual impairments to supplement (or replace) traditional driver's education instruction. During this venture, I became aware of the discrepancy between what we teach as educators to prepare youth with visual impairments to transition successfully and how this does little to help them against public perception of what constitutes "humanity" and their treatment as less-than-human. The practices and plans of advocacy teacher educators and professionals within the field of visual impairments preach as a discipline are not going to change the discrimination of people with visual impairments during their transition to adult and work life. What I now see is a broad gap in our discipline between the pedagogical practices we are using and what we need to help society realize in terms of how hard people with visual impairments are working to be accepted as able and highly competent. As noted earlier, over half the population of noninstitutionalized adults with VI ages 21-64 are unemployed (56.3\%; Erickson, Lee, \& von Schrader, 2017). Clearly there is a disconnect between how we are preparing young adults with LV/B and what they are able to accomplish due to societal norms despite being highly educated, highly trained, and highly competent. In addition to the work being done teaching students to become self-determined, autonomous individuals capable of exercising agency, professionals who work with people with visual impairments must also focus our attention on overcoming the societal roadblocks and attitudes about disability as opposed to attitudes of ability, which is what Kim (2015) is arguing in her theory of unbecoming human.

In this section, I present what my analysis of participant data might mean to the field of visual impairment, some ideas about what this analysis seems to expose about society as a whole and offer what I see as the implications of these findings. Next, I offer recommendations to 
practitioners working with adolescents with $\mathrm{LV} / \mathrm{B}$ and to researchers who look further to enhance the lives of people with visual impairments through their research endeavors. Finally, I close by suggesting future research initiatives that may address driver's education, nondriving, and advocation for individuals with VI.

\section{Findings and Implications}

\section{Perceptions of Incapacity}

Discrimination is not just limited to a single generation, reserved for those who subscribe to pre-Civil Rights era ideals about the capacity of people with disabilities. It is a real and pervasive disease that is indicative of the value placed on ability and capacity by able-bodied people that spans age groups and disciplines. By underestimating the skills, knowledge, and agency of people with visual impairments, the general public is discounting a subsection of the population that has the potential greatly to contribute and enhance society with varied experiences and inclusive perspectives.

The implications of the able-bodied mindset that people with visual impairments lack ability and capacity is important to specialists working with students with visual impairments and society at large because it has the potential to alter the way in which we educate not just people with VI, but other marginalized groups as well. Perceived incapacity of individuals with disabilities is a real and present issue. Young adults with visual impairments were the only population interviewed for this study, however, discrimination and exclusion are not limited to this group. Education about accommodations acts as the prescription for "leveling the playing field" and "rising above" one's situation to experience success as an adult. But, what if special educators and policymakers are wrong? No matter how well educators prepare youth with disabilities to transition from school to adulthood (Test et al., 2012), we can neither account for 
oppressive perspectives and discrimination they will encounter nor expect every student who faces oppression to know how to, have the resources to, or be emotionally able to "push through" those experiences. As a profession, we need deeply to examine the ways in which we prepare youth with disabilities in anticipation of able-bodied people perceiving them as incapable of contributing within shared spaces.

Society as a whole must also be made aware of its own perception of incapacity and the ways in which attitudes have a lasting effect on the self-efficacy, identity construction, independence, and transition outcomes of young adults with visual impairments. As noted previously, there are many parallels between racial discrimination and the discrimination of people with visual impairments. It is alarming and discouraging given their extensive training and education that young adults with VI should be viewed as anything other than highly accomplished and highly competent in an area of which they are highly educated. This perspective is based in privileging some and oppressing others. Unbecoming human (Kim, 2015) as a theory of intervention is a way in which society could begin to remove the privilege and value placed on capacity in order fully to value the contributions of people with visual impairments. We are currently in the most technologically rich moment in human history where access is made available to people with visual impairments with "off the shelf" technology like it has never been in the past. Regardless of this fact, access to this technology is not changing societal perceptions of incapacity and does nothing to challenge oppression and discrimination. Such a lack of change is starkly apparent in participant descriptions of experiences of discrimination emanating from peers and supervisors, in particular, despite access to technology that allows the visually impaired to engage in the same tasks as their sighted peers (e.g. smart phone, tablet). Maverick, for example, continues to feel compelled, if not required, to 
"overachieve" and "overlearn" use of some technological hardware and software just to be considered on the same playing field as his sighted employers and those competing against him for a job. Unbecoming human (Kim, 2015) is important because it brings to light the difference between equity and equality. People with visual impairments have equal access to physical environments, technology, and other social spheres, however, equity cannot and will not be reached until we begin to challenge the oppressive societal systems that are currently in place.

The implications of this research indicate that some members of society continue to hold similar perceptions of the disability characteristics of people with visual impairments as were reported in the 1970s and 1980s (Clayton, 1983; Monbeck, 1973). These attitudes of incapacity based on visual impairment have the potential to create generations of learned helpless adults with visual impairments who struggle to realize independence.

\section{Driving as a Metaphor for Ableism}

Driving as a rite of passage and transition to adulthood is exposed by participants as a metaphor for ableism. Young adults with visual impairments are all too aware of the ways in which some members of society view them. Even though driving is viewed as a right entitled to all, the process to acquire a driving license is selective and exclusionary. People are excluded based on mental, physical, and sensory abilities. The lack of entry into this idealized "club" can magnify feelings of loss and mourning when a person experiences vision loss because they have to change the way in which they engage with the world from a travel perspective as well as their expectations for the kinds of freedom of movement and spontaneity they will experience as adults. Driving and driver's education also challenge how people perceive what it means to be an adult, be independent, and develop self-efficacy. These attitudes work against people with visual 
impairments because driving is viewed as the path by which people achieve independence, responsibility, and adulthood.

Recently I presented at a conference to a room of TVIs and COMS about the ways in which we are educating students with visual impairments about concepts typically taught in driver's education. I gave an informal poll on the existence of nondriving curricula that

specifically addresses the mechanical transportation needs of people with visual impairments and the social-emotional implications that come with being a nondriver (e.g. Finding Wheels, Going Places). Of approximately 30 professionals who work with people with visual impairments, only three were aware the curricula existed, only two of those three used a few components of Finding Wheels (Corn \& Rosenblum, 2000) for instruction, and none had read the available research about the perceptions of driving and nondriving conducted over a decade ago (Corn \& Rosenblum, 2002; Corn \& Sacks, 1994; Rosenblum \& Corn, 2002a, 2002b; Sacks \& Rosenblum, 2006). There are generations of practitioners who do not realize that there exist resources to address alternatives to driving and through exposure to my presentation, there is evidence of the need in the discipline for an update to this curriculum. Through my research study, I seek to inform the discipline and contribute in a way that has not been addressed previously by investigating the experiences of young adults with visual impairments who participated in driver's education courses. I hope that attention to this issue will result in pedagogical changes and an update to the available curricula that address this topic.

\section{School Pushout}

Youth with visual impairments are not just excluded from driving, in some cases, they are excluded from the public education system. I posit that schools may be pushing students with VI to go to residential schools because educating them is expensive and many are unfamiliar with 
the ways to educate this low-incidence area. Oftentimes, services include employing a TVI and a COMS, providing and instructing in the use of assistive technology, and creating/supplying braille/large print materials, to name a few. Based on my participants' responses, I suspect it is possible that by not providing the resources necessary fully to educate a student with a visual impairment, schools may be engaging in "pushing out" these students as a means to preserve resources for able-bodied students. Four participants describe a lack of resources and support available to them in their home school districts which resulted in them electing to attend the residential school for the visually impaired. Eve Tuck, in her 2012 study, examines the relationships between various levels of school policies, the use and over-use of the General Education Development (GED) diploma, and school pushout of students of color in New York City. As with other social theorists used in my analysis, Tuck's (2012) description of "pushout" can be applied to the experiences of young adults with visual impairments. Tuck defines "pushout" as:

...the experiences of those youth who have been compelled to leave school by people or factors inside school such as disrespectful treatment from teachers and other school personnel, violence among students, arbitrary school rules, and the insurmountable presence of high stakes testing. $(2012$, p. 1)

In Tuck's research, she questions whether students of color are being pushed toward an equal or unequal credential. I ask the same about the service delivery model we offer students with visual impairments: is instruction at the residential school for the visually impaired equal to that provided in the public school? The critique of residential schools for the visually impaired is that some lack the academic rigor encountered in public schools to prepare youth for transition to adulthood. If adolescents with visual impairments are not held to same high academic 
expectations as their sighted peers and instead are receiving watered-down instruction in order to avoid accommodations or to obtain a passing grade, this could mean schools are producing unqualified and ill-prepared youth with visual impairments as a result. The other side of this coin is that neoliberalism ("inserting market values into a non-market sector of human activity;" Tuck, 2012, p. 16) is what deprives public schools of needed resources, not a concerted effort to pushout students with disabilities. Regardless of the motivation, residential schools sometimes work as an "escape hatch" from hostile school environments for adolescents with visual impairments. Amelia, Maverick, Nolan, and Tommy all recount that a lack of resources, unsupportive attitudes (administrator and teacher), and maltreatment by staff and students were what motivated them to seek refuge in the residential school for the visually impaired. There, they were equipped with the skills necessary to prepare them for life after secondary school. I cannot speak to the rigor of the academics, but I can speak to the results: four of four graduates are either attending or have graduated from college.

Despite the weight placed on perceived capacity and how perception of humanity may or may not result in pushout of students with visual impairments, I want to highlight the positive outcomes (transition, identity as independent travelers, desires; Tuck, 2009) so as not to portray this population as broken but that society at large is broken and needs intervention for the way it views and marginalizes people with visual impairments. The issue with research, for many practitioners, is that it often lacks application in the real world. I chose not to include Tuck's theoretical concept of "damage-centered research" as a concept within my theoretical frame because, while I feel comfortable drawing an inference, I cannot directly evidence an analytic claim with my current data set. Through my dissertation study, while I have exposed the ways in which people with visual impairments are perceived by the sighted world and the effects of these 
perceptions, I have also learned the most salient aspects of driver's education for youth with visual impairments. In the following sections I outline recommendations for practitioners and directions for future research as this dissertation serves as a jumping off point to a multitude of opportunities.

\section{Recommendations for Practice}

As noted previously, during the dissertation process my focus as a researcher shifted significantly away from evidencing a series of concepts in order to create a concrete list for practitioners, and toward conducting critical, emancipatory research centering systemic oppression. This shift resulted in my looking more critically at the ways in which societal perceptions are changing in regard to people with disabilities. Research investigating employers' attitudes toward people with disabilities (Unger, 2002) and the value of hiring, retaining, and promoting individuals with disabilities (Hartnett, Stuart, Thurman, Loy, \& Batiste, 2011) indicate activists, educators, and other supporters are affecting positive societal change among perceptions of capacity, agency, and independence, and in favor of people with disabilities in the workplace. This societal change is also expanding to higher education, a setting that has historically been available mainly to white, affluent, able-bodied individuals. Colleges and universities are re-evaluating who is deemed "appropriate" for higher education (Eckes \& Ochoa, 2005; Griffin, Summer, McMillan, Day, \& Hodapp, 2012), evidenced by the upcropping of programs to support the unique needs of individuals with intellectual disabilities, learning disabilities, Autism Spectrum Disorder, and many other disability categories (Eckes \& Ochoa, 2005; Griffin, Summer, McMillan, Day, \& Hodapp, 2012). The Institute for Community Inclusion at the University of Massachusetts Boston, among other large universities, has spent the past 50 years researching, educating and providing support to ensure access for people with 
disabilities to health care, education, employment, and community life (Institute for Community Inclusion, 2019). While many researchers have come before me to challenge and inspire change in societal perceptions of people with specific disabilities, my induction into this movement occurred more recently. In the area of LV/B, advocacy groups such as the National Federation of the Blind (Kornbluh, 2011) and the American Council of the Blind (Bentzen, Barlow, Franck, 2000) have worked tirelessly through lobbying efforts on Capitol Hill to ensure people with visual impairments are represented and included in all aspects of society. These organizations' work has laid the legislative foundation for researchers and activists practically to build upon. The National Industries for the Blind and AbilityOne have also worked to change inaccurate and outdated perceptions of capacity and combat the unemployment rate for people with vision loss and other significant disabilities by connecting with various nonprofit agencies to employ people who are blind or visually impaired in stable, government jobs (National Industries for the Blind, 2019; U.S. AbilityOne Commission, 2019). My hope is that my research and recommendations for practice will support and contribute to the common goal of equity for people with LV/B.

\section{TVIs and COMS in PK-12 Schools}

Running up against nondriving and driver's education as an educator for students with visual impairments is inevitable. American society is focused on autonomy and one of the ways in which we exhibit autonomy is through driving. Many school districts require participation in the classroom portion of driver's education as a means to earn credit toward graduation. Instead of acting as a blanket requirement, students with visual impairments should be afforded the opportunity to choose whether they partake in driver's education coursework instead of having it imposed on them. Candid and honest conversations must be had with the student and his family regarding whether driver's education is important to his future life goals. If a decision is made in 
favor of driver's education, the TVI or COMS should act as a conduit between the student/family and the driver's education staff/administration to support the school in providing meaningful instruction to the adolescent with a visual impairment. For those whose vision is close to the cutoff for obtaining a driver's license, taking driver's education may benefit them. Much like an IEP team creates a plan based on the individual's needs, participation in driver's education should be treated with the same consideration but without shaming students if they choose to opt out of the course.

Another solution to the "driver's education requirement" could be to provide an alternative to driver's education where students engage in disability-specific instruction that will aid in positive transition outcomes, including positive identity creation and self-determination. This may include O\&M that incorporates concepts discussed in driver's education but that are relevant to an adolescent with a visual impairment as an independent traveler or passenger in a car, and that focuses on individuals' strengths. My own study is definitional in nature and I make no claim to generalizability, so significant additional research will be required in order to build a new curriculum or build upon a curriculum that already exists. If I were to devise this curriculum, I would utilize the expertise of people like my participants to create a real and meaningful solution and connect to life experiences as they relate to their encounters with discrimination so they may experience empowerment with which to affect positive change. Additionally, I would seek to bridge the gap between educating adolescents with visual impairments, who tend to be "siloed" during one-on-one instruction, and their same-age peers by facilitating understanding through shared experiences and appreciation for differing means of independent travel. One of the ways I would do this is by providing experiential leaning opportunities to sighted adolescents so they may understand what travelers with visual 
impairments learn to make these students authentically a part of the same world. This might include teaching lessons within classrooms where all students learn about listening and the types of clues listening provides to a driver and pedestrian. Another option might be to invite sighted students to participate in an O\&M lesson while blindfolded. Again, much more work will need to be conducted to build an alternative to driver's education for youth with visual impairments which places primary focus on their strengths (Yosso, 2005). Based on participants' responses, such an offering will likely include some of the salient concepts I describe next.

Participants' major takeaways from driver's education coursework were the following: how to identify reckless driving behavior (who to ride with and who not to ride with); pedestrians have the "right of way;" location of controls in the vehicle (e.g. levers, gear shifts); meanings of street signs and markings; and the cognitive load placed on the driver. They did not, however, learn much by way of pedestrian-focused instruction, especially concerning pedestrians with disabilities (e.g. White Cane Law, what to do if you are at an intersection with a white cane or wheelchair user). In terms of the perceived value in taking driver's education, many tried to "make the best of the situation," as it was a requirement for graduation. They were disappointed by scant discussion about pedestrian safety and were surprised that even though they were in the class, no mention was made about the White Cane Law. Participants also did not feel engaging in the driver's education course informed their independent travel in a profound way. Instead, consistent O\&M instruction was a recurring theme as the main source of their proficiency in independent travel.

\section{Researchers of People with Visual Impairments}

The last recommendation I offer is based in social theory. My data analysis raised many more questions than it answered. Should we be educating young people with visual impairments 
about the social issue of having a disability? What is the role of activism in their education and future professional careers? Through education, should we be grooming activists and making them aware of their political power so they can take up the cause of changing hearts and minds to consider public perception of individuals with disabilities? Kim's (2015) idea of unbecoming human is highly theoretical, challenging what humanity is to bring more people into the fold of normalcy. To speak to the ways in which educators and society can apply this theory to change hearts and minds is beyond the scope of the present study. Based on the findings of this study, I better understand the types of questions that would need to be posed in order to come closer to application of this theory. As Kim (2015) prescribes, we cannot just take power from one and give it to another because it reinforces the same inequitable structure. Instead, society should consider what changes would need to occur to make a notion like "unbecoming human" practical. What might incentivize people to relinquish their subjectivities? How do we alter the system without creating new inequities? How can we approach the ideas Kim espouses as a discipline to make them practically meaningful for people with VI and people who live and work with them? These questions are the beginnings of a seismic shift in the way we think about people with LV/B and also how we think about disabilities as a whole.

\section{Directions for Future Research}

\section{For Practitioners}

Much more research is needed with different populations in different states to determine if the recommendations and value placed on certain components of driver's education are meaningful to all people with visual impairments. This could be achieved through development and participation in a Delphi study to reach consensus among certified orientation and mobility specialists and adult travelers with visual impairments. Additionally, folks with visual 
impairments who have not taken driver's education, are unemployed, and in different age groups could be interviewed to better define concepts for practitioners.

To improve practice among TVIs and COMS, I would like to develop online modules that specifically address the independent travel needs of youth and young adults with visual impairments while incorporating relevant O\&M skills and concepts typically taught in driver's education. These actions would also be benefited by the update of existing curricula (Going Places, Finding Wheels, Reclaiming Independence) to reflect changes in technology and the built environment. I seek to contribute a variety of resources to provide students with visual impairments and their families alternatives to participation in driver's education when it is not appropriate that is meaningful and applicable to their transition experiences.

\section{Application of Social Theory}

Moving forward with this line of inquiry, I would like to review this data through an alternative theoretical lens, specifically I am interested in employing Eve Tuck's theory of damage-centered research (2009). Tuck (2009) explores the implications of damage-centered research in an open letter to Native American and urban communities, researchers, and educators. Predicated on a flawed theory of change, Tuck's concept of damage-centered research is based on well-meaning and even critically conscious researchers' attempts to achieve a form of reparations by using their research findings to appeal to the guilt of the oppressors. Rather than working from a strengths-based perspective, such research findings instead paint participants and communities as "broken" or lacking (Tuck, 2009) even when well-meaning researchers seek to obtain resources to "help" that community. The pernicious result of such practice is a community that takes on the identity as "damaged," "broken," and lacking, which in turn affects the way in which the general public views these communities. Damage-centered 
research may, in part, explain why marginalized groups have struggled to change the hearts and minds of their oppressors; if they view themselves as damaged, then it is likely others will view them as damaged and lacking capacity as well. I argue use of such a frame to approach research with the visually impaired this could assist in providing findings upon which I might develop a program of advocacy. A strengths-based approach to research may be used to educate professionals about the dangers of deficit thinking and see the capabilities and identities of young people and see them beyond their disability, and move away from the colonizing effects of "scientifically" naming individuals and communities as "broken."

Additionally, I foresee use of paperson's (2010) theory on urban education and postcolonialism as it is related closely both to my data and to Tuck's theoretical work. In hir (paperson's pronoun of choice) article, paperson (2010) presents facts supporting the continued existence (and pursuit) of colonialism in the United States and dives deeply into the interplay between imperialism and colonialism in U.S. public schools. Most often exemplified in public schools dominated by students of color, colonial schooling is used as a tool to maintain control and oppression of marginalized groups. Imperial education, an overarching, ancient method of control, is the means by which people who have been "othered," but who seek the promise of inclusion, learn to be part of a majority white, able-bodied culture.

paperson (2010) proposes a framework that investigates the ways in which colonial schooling and imperialist education act as forces upon three different aspects of empire: (a) metropole, (b) ghetto, and (c) colony. Shifting abruptly away from the notion of ghetto as place, paperson (2010) defines "ghetto" as a "dislocating procedure of othered people, not a sociological space" (p. 10). The term "ghetto" has historically been used to describe a physical location where people who have been methodically pushed out of majority society are dislocated. 
I am convinced of the utility of such a theoretical concept to my future work in LV/B research because I view strong parallels between the space of dislocation ("ghetto") experienced by people of color and those experienced by people with disabilities. I foresee investigating how imperialist schooling creates docile bodies (Foucault, 1977) and how schooling offers those with visual impairments investment in the larger empire by hard-selling the false promise of inclusion. Once lulled into docility, rather than "inclusion," dislocated people experience instead a methodical "pushing out" of certain ethnic groups; I see a strong parallel to the "pushing out" of people with visual impairments in educational and professional settings.

Finally, I would like to reexamine the rich, family-level participant data I collected. Given the chosen focus of my dissertation analysis and findings, I was unable to include family participants' data in this round of analysis. Reentering the field to conduct additional family member interviews based on data analysis completed through my existing theoretical lens and engaging in another theoretical frame is likely to yield compelling analytic claims and findings that deepen and magnify current data analysis and findings. 


\section{REFERENCES}

Albrecht, G., \& Devlieger, P. (1999). The disability paradox: High quality of life against all odds. Social Science \& Medicine, 48(8), 977-988. doi: 10.1016/S0277-9536(98)00411-0

Albrecht, G. L., Seelman, K., \& Bury, M. (2001). Handbook of disability studies. Thousand Oaks, CA: SAGE. doi: 10.4135/9781412976251

Armstrong, J. (2010). Naturalistic inquiry. In N. J. Salkind (Ed.), Encyclopedia of research design (pp. 881-885). Thousand Oaks, CA: SAGE. doi: 10.4135/9781412961288.n262

Bandura, A. (1977). Self-efficacy: Toward a unifying theory of behavioral change. Psychological Review, 84, 191-215.

Barraga, N. (1983). Visual handicaps and learning (Rev. ed.). Austin, TX: Exceptional Resources.

Bentzen, B., Barlow, J., Franck, L. (2000). Addressing barriers to blind pedestrians at signalized intersections. Institute of Transportation Engineers Journal, 70(9), 32-35.

Bevan-Brown, J., \& Walker, T. (2013). Taking culture into account: A Māori perspective on visual impairment. Journal of Visual Impairment \& Blindness, 107(5), 388-392.

Blackorby, J., \& Wagner, M. (1996). Longitudinal postschool outcomes of youth with disabilities: Findings from the national longitudinal transition study. Exceptional Children, 62, 399-413.

Brooks, J. O., Mossey, M. E., Tyler, P., \& Collins, J. C. (2013). An exploratory investigation: Are driving simulators appropriate to teach pre-driving skills to young adults with intellectual disabilities? British Journal of Learning Disabilities, 42, 204-213. doi: 10.111/bld.12029 
Brune, J. A., \& Wilson, D. J. (2013). Disability and passing: Blurring the lines of identity. Philadelphia, PA: Temple University Press

Campbell, F. A. (2008). Exploring internalized ableism using critical race theory. Disability \& Society, 23(2), 151-162. doi: 10.1080/09687590701841190

Chung, E. L. (1996). Asian Americans. In M. C. Julia (Ed.), Multicultural awareness in the health care professions (pp. 77-110). Boston, MA: Allyn \& Bacon.

Clayton, I. (1983). Career preparation for the visually handicapped. Education of the Visually Handicapped, 14, 115-120.

Corn, A. L., \& Rosenblum, L. P. (2000). Finding wheels: A curriculum for nondrivers with visual impairments for gaining control of transportation needs. Austin, TX: Pro-Ed.

Corn, A. L., \& Rosenblum, L. P. (2002). Experiences of older adults who stopped driving because of their visual impairments: Part 2. Journal of Visual Impairment \& Blindness, $96(7), 485-500$.

Corn, A., \& Sacks, S. (1994). The impact of nondriving on adults with visual impairments. Journal of Visual Impairment \& Blindness, 88(1), 53-69.

Council for Exceptional Children. (2009). What every special educator must know: Ethics, standards, and guidelines ( $6^{\text {th }}$ ed.). Arlington, VA: Council for Exceptional Children.

Crotty, M. (1998). The foundations of social research: Meaning and perspective in the research process. Washington, DC: SAGE.

Dweck, C. S. (1975). The role of expectations and attributions in the alleviation of learned helplessness. Journal of Personal and Social Psychology, 31, 674-685. 
Dweck, C. S., \& Licht, B. G. (1980). Learned helplessness and intellectual achievement. In J. Garber \& M. E. P. Seligman (Eds.), Human helplessness: Theory and applications (pp. 197-222). New York, NY: Academic Press.

Dworkin, A. (1981). Pornography: Men possessing women. New York, NY: G. P. Putnam’s Sons.

Eckes, S., \& Ochoa, T. (2005). Students with disabilities: Transitioning form high school to higher education. American Secondary Education, 33(3), 6-20.

Education for All Handicapped Children Act of 1975, 20 U.S.C. §1401-1420 (2016).

Eisenhelder, S. (1990). The asphalt identikit: Old age and the driver's license. International Journal on Aging and Human Development, 30, 14.

Erikson, E. H. (1950). Childhood and society. New York, NY: W. W. Norton.

Erickson, W., Lee, C., \& von Schrader, S. (2017). Disability statistics from the 2014 American Community Survey (ACS). Ithaca, NY: Cornell University, Yang Tan Institute (YTI). Retrieved from http://disabilitystatistics.org/reports/acs.cfm?statistic $=2$

Fearon, J. (1999). What is identity (As we now use the word?). Unpublished manuscript, Stanford University, Stanford, CA. Retrieved from https:/web.stanford.edu/group/fearon-research/cgi-bin/wordpress/wpcontent/uploads/2013/10/What-is-Identity-as-we-now-use-the-word-.pdf

Fleming, J., \& Ledogar, R. (2008). Resilience, and evolving concept: A review of literature relevant to aboriginal research. Pimatisiwin, 6(2), 7-23.

Forber-Pratt, A. J., \& Zape, M. P. (2016). Disability identity development model: Voices from the ADA-generation. Disability and Health Journal, 10(2), 350-355. 
Fordyce, W. (1971). Behavioral methods in rehabilitation. In W. Neff (Ed.), Rehabilitation psychology. Washington, DC: American Psychological Association.

Foucault, M. (1977). Discipline and punish: The birth of the prison. New York, NY: Vintage.

Freire, P. (2015). Pedagogy of the oppressed. New York, NY: Bloomsbury Academic.

Glaser, B., \& Strauss, A. (2012). The discovery of grounded theory: Strategies for qualitative research. New Brunswick, NJ: Transaction.

Gomez, L. E., Peña, B., Arias, B., \& Verdugo, M. A. (2014). Impact of individual and organization variables on quality of life. Social Indicators Research, 125(2), 1-16. doi: $10.1007 / \mathrm{s} 11205-014-0857-6$

Griffin, M., Summer, A., McMillan, E., Day, T., \& Hodapp, R. (2012). Attitudes toward including students with intellectual disabilities at college. Journal of Policy and Practice in Intellectual Disabilities, 9(4), 234-239.

Hartnett, H., Stuart, H., Thurman, H., Loy, B., \& Batiste, L. (2011). Employers’ perceptions of the benefits of workplace accommodations: Reasons to hire, retain and promote people with disabilities. Journal of Vocational Rehabilitation, 34, 17-23. doi: 10.3233/JVR$2010-0530$

Hasazi, S. B., Gordon, L. R., \& Roe, C. A. (1985). Factors associated with the employment status of handicapped youth exiting high school from 1979 to 1983. Exceptional Children, 51, 455-469.

Hatlen, P. (2000). Historical perspectives. In A. J. Koenig \& M. C. Holbrook (Eds.), Foundations of education: History and theory of teaching children and youths with visual impairments (vol. 1) (2 $2^{\text {nd }}$ ed.) (pp. 1-54). New York, NY: AFB. 
Hatlen, P. (2003, December 4-7). Impact of literacy on the expanded core curriculum. Paper presented at the Getting in Touch with Literacy conference, Vancouver, British Columbia, Canada.

Hatt, B. (2012). Smartness as a cultural practice in schools. American Educational Research Journal, 49(3), 438-460. doi: 10.3102/0002831211415661

Head, D., Pysh, M., Chalfant, J., \& Spencer, R. (1992). Learned helplessness in children with visual handicaps: A pilot study of expectations, persistence, and attributions. Phoenix, AZ: Arizona State Department of Education.

HeavyRunner, I., \& Marshall, K. (2003). Miracle survivors: Promoting resilience in Indian students. Tribal College Journal, 14(4), 14-19.

Helms, J. (1997). Toward a model of White racial identity development. In K. Arnold \& I. King (Eds.), College student development and academic life: Psychological, intellectual, social and moral issues (pp. 49-66). New York, NY: Garland.

Hyun, J. K., \& Fowler, S. A. (1995). Respect, cultural sensitivity, and communication. Teaching Exceptional Children, 28(1), 25-28.

Individuals with Disabilities Education Act of 1990, 20 U.S.C. § 1400 et seq. (2016).

Individuals with Disabilities Education Act of 1997, 20 U.S.C. § 1400 et seq. (2016).

Individuals with Disabilities Education Improvement Act of 2004, 20 U.S.C. § 1400 et seq. (2016).

Institute for Community Inclusion. (2019). About the institute for community inclusion. Retrieved from https://www.communityinclusion.org/project.php?project_id=35 
Jenaro, C., Verdugo, M. A., Caballo, C., Balboni, G., Lachapelle, Y., \& Otrebski, W. (2005). Cross-cultural study of person-centered quality of life domains and indicators. Journal of Intellectual Disability Research, 49, 734-739. doi: 10.1111/j.1365-2788.2005.00742.x

Jones, S. R., \& McEwen, M. K. (2000). A conceptual model of multiple dimensions of identity. Journal of College Student Development, 41(4), 405-414.

Kef, S. (1997). The personal networks and social supports of blind and visually impaired adolescents: Part 1. Journal of Visual Impairment \& Blindness, 91, 236-239.

Kelly, M., \& Millward, L. (2004). Identity and illness. In. D. Kelleher \& G. Leavey (Eds.), Identity and health (pp. 1-18). New York, NY: Routledge.

Kim, E. (2015). Unbecoming human: An ethics of objects. GLQ, 21(2-3), 295-320. doi:10.1215/10642684-2843359

Klausner, S. Z. (1969). Disabled families: A study of a link between the social contributions of the disabled and the retardation of their rehabilitation in the family context. Philadelphia, PA: Center for Research on the Acts of Man.

Koestler, F. A. (1976). The unseen minority: A social history of blindness in the United States. New York, NY: D. McKay Co.

Kornbluh, F. (2011). Disability, antiprofessionalism, and civil rights: The National Federation of the Blind and the "right to organize" in the 1950s. Journal of American History, 97(4), $1023-1047$.

Landmark, L. J., Ju, S., \& Zhang, D. (2010). Substantiated best practices in transition: Fifteenplus years later. Career Development for Exceptional Individuals, 33, 165-176.

Large, T. (1982). The effects of attitudes upon the blind: A reexamination. Journal of Rehabilitation, 6, 33-35. 
Lincoln, Y., \& Guba, E. (1985). Naturalistic inquiry. Beverly Hills, CA: SAGE.

Lindauer, O. (1998). Archaeology of the Phoenix Indian School. Archaeology, 51(2). Retrieved from https://archive.archaeology.org/online/features/phoenix/

Lipkowitz, S., \& Mithaug, D. E. (2003). Assessing self-determination prospects of students with different sensory impairments. In D. E. Mithaug, D. K. Mithaug, M. Agran, J. E. Martin, \& M. L. Wehmeyer (Eds.), Self-determined learning theory: Construction, verification, and evaluation (pp. 104-120). Mahwah, NJ: Lawrence Erlbaum.

Lowenfeld, B. (1973). Psychological considerations. In B. Lowenfeld (Ed.), The visually handicapped child in school (pp. 27-60). New York, NY: John Day.

Luthar, S. (2006). Resilience in development: A synthesis of research across five decades. In D. Cicchetti \& D. Cohen (Eds.), Developmental psychopathology: Risk, disorder, and adaptation (pp. 740-795). New York, NY: Wiley.

Malian, I., \& Nevin, A. A. (2002). Review of self-determination literature: Implications for practitioners. Remedial and Special Education, 23(2), 68-74.

Marcia, J. (1966). Development and validation of ego-identity status. Journal of Personality and Social Psychology, 3(5), 551-558.

Marcia, J. (1980). Identity in adolescence. In J. Adelson (Ed.), Handbook of adolescent psychology (pp. 159-187). New York, NY: Wiley \& Sons.

Marcia, J. (2002). Identity and psychosocial development in adulthood. Identity: An International Journal of Theory and Research, 2(1), 7-28.

Mathison, S. (1988). Why triangulate? Educational Researcher, 17(2), 13-17. 
McDonnall, M. C. (2011). Predictors of employment for youths with visual impairments: Findings from the second National Longitudinal Transition Study. Journal of Visual Impairment \& Blindness, 105, 453-466.

McDonnell, J., Wilcox, B., \& Boles, S. M. (1986). Do we know enough to plan for transition? A national survey of state agencies responsible for services to persons with severe handicaps. Journal of the Association for Persons with Severe Handicaps, 11, 53-60.

McGill, T., \& Vogtle, L. K. (2001). Driver's education for students with physical disabilities. Exceptional Children, 67(4), 455-466.

Meier, B. (1992, May 10). Safety of older drivers: A growing concern for regulators. Austin American-Statesman, 17.

Miyagawa, S. (1999). Journey to excellence: Development of the military and VA blind rehabilitation programs in the $20^{\text {th }}$ century. Lakeville, MN: Galde.

Monbeck, M. (1973). The meaning of blindness. Bloomington, IN: Indiana University Press.

Moore, J. E. (1984). Impact of family attitudes toward blindness/visual impairment on the rehabilitation process. Journal of Visual Impairment \& Blindness, 78(3), 100-106.

Mpofu, E., \& Harley, D. (2006). Racial and disability identity: Implications for the career counseling of African Americans with disabilities. Rehabilitation Counseling Bulletin, 50(1), 14-23. doi: 10.1177/00343552060500010301

National Industries for the Blind. (2019). About National Industries for the Blind. Retrieved from https://www.nib.org/about/ 
Noonan, B., Gallor, S., Hensler-McGinnis, N., Fassinger, R., Wang, S., \& Goodman, J. (2004). Challenge and success: A qualitative study of the career development of highly achieving women with physical and sensory disabilities. Journal of Counseling Psychology, 51(1), $68-80$.

Otto, S. (2007). Beneath and beyond truth: Studying literary narratives to research human phenomena. International Journal of Research and Method in Education, 30(1), 73-87.

Otto, S. (2017). Theoretical perspectives summary sheet. EAF 515: Methods of qualitative inquiry (pp. 1-2). Normal, IL: Illinois State University.

Otto, S., \& Gunzenhauser, M. G. (2006, April). “Disciplined imagination” and other oxymorons: Teaching qualitative data analysis. Paper presented at the annual meeting of the American Educational Research Association, San Francisco, CA.

Otto, S., \& Gunzenhauser, M. G. (2014, April). “Disciplined imagination” and other oxymorons, part II: Exploring the continuing vagaries of teaching qualitative data analysis. Paper presented at the annual meeting of the American Educational Research Association, Philadelphia, PA.

Pang, V. O. (1995). Asian American children: A diverse population. In D. T. Nakanishi \& T. Y. Nishida (Eds.), The Asian American educational experience: A source book for teachers and students (pp. 167-179). New York, NY: Routledge.

paperson, 1. (2010). The postcolonial ghetto: Seeing her shape and his hand. Berkeley Review of Education, 1(1), 5-34.

Patrick, D. L. (1997). Rethinking prevention for people with disabilities part I: A conceptual model for promoting health. American Journal of Health Promotion, 11, 257-260. 
Patton, M. (2002). Qualitative research and evaluation methods ( $3^{\text {rd }}$ ed.). Thousand Oaks, CA: SAGE.

Peterson, K., (1992, December 14). Teens face issues but driver's license comes first. U.S. Today, 1.

Ponchillia, P., \& Ponchillia, S. (1996). Foundations of rehabilitation teaching with persons who are blind or visually impaired. New York, NY: AFB.

Price, L. A., Wolensky, D., \& Mulligan, R. (2002). Self-determination in action in the classroom. Remedial and Special Education, 23(2), 109-115.

Roberts, E. (1977). On disability rights. World Institute on Disability Founders. Retrieved from https://wid.org/resources/wids-founders/

Rosenblum, L. (2004). Going places. Winnetka, IL: Hadley School for the Blind.

Rosenblum, L., \& Corn, A. (2002a). Experiences of older adults who stopped driving because of their visual impairments: Part 1. Journal of Visual Impairment \& Blindness, 96(6), 389398.

Rosenblum, L., \& Corn, A. (2002b). Experiences of older adults who stopped driving because of their visual impairments: Part 3. Journal of Visual Impairment \& Blindness, 96(10), 701710.

Sacks, S. Z., \& Rosenblum, L. P. (2006). Adolescents with low vision: Perceptions of driving and nondriving. Journal of Visual Impairment \& Blindness, 100(4), 212-222.

Sacks, S. Z., Wolffe, K. E., \& Tierney, D. (1998). Lifestyles of students with visual impairments: Preliminary studies of social networks. Exceptional Children, 64, 463-478. 
Sapp, W., \& Hatlen, P. (2010). The expanded core curriculum: Where we have been, where we are going, and how we can get there. Journal of Visual Impairment \& Blindness, 104(6), $338-348$.

Schalock, R. L., \& Verdugo, M. Á. (2002). Handbook on quality of life for human service practitioners. Washington, DC: American Association of Mental Retardation.

Schalock, R., Verdugo, M., Jenaro, C., Wang, M., Wehmeyer, M., Xu, J., \& Lachapelle, Y. (2005). Cross-cultural study of quality of life indicators. American Journal on Mental Retardation, 110(4), 298-311. doi:

10.1352/08958017(2005)110[298:CSOQOL]2.0.CO;2

Schlecter, T. M., \& Gump, P. V. (1983). Car availability and the daily life of the teenage male. Adolescence, 69, 101-113.

Sebald, H. (1983). Life in the subculture. In J. F. Cuber \& A. C. Clarke (Eds.), Adolescence: A sociological analysis (pp. 255-262). New York, NY: Appleton Century-Crofts.

Shaw, A., Gold, D., \& Wolffe, K. (2007). Employment-related experiences of youths who are visually impaired: How are these youths faring? Journal of Visual Impairment \& Blindness, 101(1), 7-21.

Sheffield, R. (2014). Qualitative investigation of the experience of quality of life for young adults who are blind (Unpublished doctoral dissertation). Texas Tech University, Lubbock, TX.

Test, D. W., Mazzotti, V. L., \& Mustian, A. L. (2012). Leadership for transition to postsecondary settings. In J. B. Crockett, B. S. Billingsley, \& M. L. Boscardin (Eds.), Handbook of Leadership and Administration in Special Education (pp. 337-357). New York, NY: Routledge. 
Tringo, J. (1970). The hierarchy of preference toward disability groups. Journal of Special Education, 4, 295-306.

Tuck, E. (2009). Suspending damage: A letter to communities. Harvard Educational Review, 79(3), 409-427.

Tuck, E. (2012). Urban youth and school pushout: Gateways, get-aways, and the GED. New York, NY: Routledge.

Unger, D. (2002). Employers' attitudes toward persons with disabilities in the workforce: Myths or realities? Focus on Autism and Other Developmental Disabilities, 17(1), 2-10.

U.S. AbilityOne Commission. (2019). AbilityOne facts. Retrieved from https://www.abilityone.gov/

Valencia, R. (2010). Dismantling contemporary deficit thinking: Educational thought and practice. New York: NY: Routledge.

Wagner, M., Marder, C., Blackorby, J., Cameto, R., Newman, L., Levine, P., \& Davies-Mercier, E. (with Chorost, M., Garza, N., Guzman, A., \& Sumi, C.). (2003). The achievements of youth with disabilities during secondary school. A report from the national longitudinal transition study-2 (NLTS2). Menlo Park, CA: SRI International.

Wang, M., Schalock, R. L., Verdugo, M. A., \& Jenaro, C. (2010). Examining the factor structure and hierarchical nature of the quality of life construct. American Journal on Intellectual and Developmental Disabilities, 115, 218-233. doi: 10.1352/1944-7558-115.3.218

Welsh, R. L. (1997). The psychosocial dimensions of orientation and mobility. In B. B. Blasch, W. R. Weiner, \& R. L. Welsh (Eds.), Foundations of orientation and mobility ( $2^{\text {nd }}$ ed) (pp. 200-224). New York, NY: AFB. 
White, H. (1985). Tropics of discourse: Essays in cultural criticism. Baltimore, MD: Johns Hopkins University Press.

Will, M. (1984, March-April). Bridges from school to working life. Programs for the Handicapped. Washington, DC: Clearinghouse on the Handicapped.

Wolfensberger, W. (1983). Social role valorization: A proposed new term for the principle of normalization. Mental Retardation, 21(6), 234-239.

Wolffe, K., \& Kelly, S. M. (2011). Instruction in areas of the expanded core curriculum linked to transition outcomes for students with visual impairments. Journal of Visual Impairment \& Blindness, 105, 340-349.

Wolffe, K. E., \& Sacks, S. Z. (1997). The social network pilot project: Lifestyles of students with visual impairments. Journal of Visual Impairment \& Blindness, 91, 245-257.

Worley, V., Otto, S., \& Bailey, L. E. (2010). Discovering the more: Reading Wright's, Cather's, and Collette's texts as philosophy of education. Educational Studies, 46(2), 192-223.

Yosso, T. (2005). Whose culture has capital? A critical race theory discussion of community cultural wealth. Race, Ethnicity and Education, 8(1), 69-91. doi:

$10.1080 / 1361332052000341006$ 


\section{APPENDIX A: LEVEL ONE SOLICITATION EMAIL (TO DIRECTORS OF UNIVERSITY/COMMUNITY COLLEGE DISABILITY SERVICES)}

Dear

My name is Molly Pasley and I am working on my doctorate in Special Education at Illinois State University. I write because I hope you might help me solicit participants for my dissertation study given your role at your institution. I plan to study young adults with visual impairments (low-vision and blind age 18-30) who live in . I want to talk to them about their experiences in Driver's Education courses during high school and how what they learned may influence their mobility, self-efficacy, etc. during young adulthood. I have ready a solicitation letter, approved by the Illinois State University Institutional Review Board, that will provide potential study participants with information on my study as well as contact information should they be interested in participating. Might you forward this information to clients with registered visual impairments at ___ ? If so, I can forward the solicitation letter to you right away. If not, I wonder if you might share recommendations for other ways I could gather potential participants for my study? I appreciate your time and expertise.

Thank you very much for your consideration. I am happy to answer any additional questions you may have and I look forward to hearing from you!

Sincerely

Molly Pasley, M.S.Ed

Illinois State University Doctoral Candidate 


\section{APPENDIX B: PARTICIPANT RECRUITMENT EMAIL}

Dear [Name],

My name is Molly Pasley and I am a doctoral candidate at Illinois State University in the Special Education department. For my dissertation research, I am conducting a study about understanding the perceived impact of driver's education on young adults with visual impairments. The purpose of my study is to examine the role of driver's education in the lives of people with visual impairments, particularly how the course affects independent travel. [Name] at [institution] has forwarded this letter and my information to potential participants. You have received this letter because you are [or are related to] a young adult with a visual impairment. I'm writing to invite you to participate in my study should you meet some additional criteria. Should you be interested in participating, I'd like to speak with you at your earliest convenience about this additional criteria and your availability for interviews.

While you are under no obligation to participate in my study, I would very much value and appreciate your input. My hope is that this research will lead to improvements in transition experiences for people with visual impairments/blindness. If you decide to participate, I will schedule an initial, 45-minute interview sometime between [DATE to be noted after approval] and June $1^{\text {st }}$. After we meet for our interview, I'll make arrangements to observe you completing everyday tasks (e.g. going to work, engaging in leisure activities, etc.). I will also want to interview you again, as a follow-up for another, approximately 45-minute interview. With your permission, I would also like to interview one or more of your family members. If you do not wish for me to speak with your family members, you can still participate in the study.

Some of the risks associated with this study are that you may become shy at the thought of being recorded or uncomfortable sharing information you know is being recorded, or you may 
experience feelings of loss and sadness when talking about your experiences with driver's education and reliving a time when you were unable to share in the same milestone as your peers. If any of these occur, you are welcome to abstain from answering questions that cause undue emotional discomfort, you may take a break, or you may opt out of the research entirely.

An additional risk is loss of confidentiality as your voice may be recognized on the digital audio recording. This risk will be minimized by the researcher using pseudonyms to protect your identity and other identifying information (town, family members). All voice recordings will also be kept in a locked filing cabinet that only the researcher has access to.

The final risk to participants is undue influence or distress in feeling pressured to provide details for a family member to participate. Providing family member information is not required to participate in this study and you can choose to withdraw at any time.

While you will gain insight into what factors led to you traveling independently through vocalizing your experiences and having the opportunity to reflect upon them during the interview process, there is no direct benefit to you associated with participation in this research study.

If you would like to participate in this study or would like additional information, I'd be more than happy to speak with you and answer your questions. Feel free to contact me by replying to this email (․maclese@,ilstu.edu) or calling me at 309-338-3716. Thank you for your time!

Sincerely,

Molly Pasley, M.S.Ed

Illinois State University Doctoral Candidate 


\section{APPENDIX C: PHONE SCRIPT WITH INCLUSIONARY QUESTIONS TO DETERMINE PARTICIPANT ELIGIBILITY}

Hello [Name]. My name is Molly Pasley and I am a doctoral candidate at Illinois State University in the Special Education department. For my dissertation research, I am conducting a study about understanding the perceived impact of driver's education on young adults with visual impairments. Before we go any further, I need ask you a few questions to determine if you meet the criteria to participate.

1. How old are you? (participants must be between 18-30)

2. Do you live in ?

3. What is your visual diagnosis?

4. Are you eligible to receive services through your state's human services department as a result of your visual impairment?

5. Do you currently hold or have you ever earned a driver's license?

6. Does your vision make you unable to drive?

7. Have you ever participated in driver's education? (must have participated in driver's education)

8. Are you comfortable with me contacting a family member to conduct an interview?

9. If you are comfortable with me contacting a family member, what is the individual's contact information? 


\section{If potential participant meets inclusion criteria:}

Fortunately, you are eligible to participate in this study. I want to remind you that participation is entirely voluntary. Do you think you might be interested in participating in this study?

If you do decide to participate in the study, the next phase is for me to meet you for a face-toface interview in a location of your choice that is convenient for you for 45 minutes. What is a day and time that is best for you?

I also would like to schedule an observation at some point following the interview but that does not need to be scheduled at this time.

Thank you very much for your willingness to participate in this research study, I look forward to chatting with you in the near future!

\section{If potential participant DOES NOT meet inclusion criteria:}

Unfortunately, [reason unable to participate] disqualifies you from participation in this study. I greatly appreciate your time in answering my questions and hope you have a wonderful [day, afternoon, evening]. 


\section{APPENDIX D: FAMILY MEMBER RECRUITMENT SCRIPT}

Hello [Name]. My name is Molly Pasley and I am a doctoral candidate at Illinois State University in the Special Education department. For my dissertation research, I am conducting a study about understanding the perceived impact of driver's education on young adults with visual impairments. The purpose of my study is to examine the role of driver's education in the lives of people with visual impairments, particularly how the course affects independent travel. [Family member] is a participant in this study and gave me your contact information. You have received this phone call because you are related to a young adult with a visual impairment. I'm calling to invite you to participate in my study.

While you are under no obligation to participate in my study, I would very much value and appreciate your input. My hope is that this research will lead to improvements in transition experiences for people with visual impairments/blindness. If you decide to participate, I will schedule a single, 45-minute interview sometime between [DATE to be noted after approval] and June $1^{\text {st }}$.

Participation in this study is entirely voluntary. If you choose to participate in this study, you are free to withdraw permission at any time. You may also decline to participate.

Some of the risks associated with this study are that you may become shy at the thought of being recorded or uncomfortable sharing information you know is being recorded, or you may experience feelings of loss and sadness when talking about your child's experiences with driver's education and reliving a time when he or she was unable to share in the same milestone as his/her peers. If any of these occur, you are welcome to abstain from answering questions that cause undue emotional discomfort, you may take a break, or you may opt out of the research entirely. 
An additional risk is loss of confidentiality as your voice may be recognized on the digital audio recording. This risk will be minimized by the researcher using pseudonyms to protect your identity and other identifying information (town, family members). All voice recordings will also be kept in a locked filing cabinet that only the researcher has access to.

The final risk to participants is undue influence or distress in feeling pressured to provide details for a family member to participate. Providing family member information is not required to participate in this study and you can choose to withdraw at any time.

While you will gain insight into what factors led to your family member traveling independently through vocalizing your experiences and having the opportunity to reflect upon them during the interview process, there is no direct benefit to you associated with participation in this research study.

Do you think you might be interested in participating in this study?

\section{If family member responds "yes" to above question:}

If you do decide to participate in the study, the next phase is for me to meet you for a face-toface interview in a location of your choice that is convenient for you for 45 minutes. What is a day and time that is best for you?

Thank you very much for your willingness to participate in this research study, I look forward to chatting with you in the near future! 


\section{If family member responds "no" to above question:}

Thank you for your consideration, I greatly appreciate your time in taking my phone call and hope you have a wonderful [day, afternoon, evening]. 


\section{APPENDIX E: FAMILY MEMBER RECRUITMENT EMAIL}

Dear [Name],

My name is Molly Pasley and I am a doctoral candidate at Illinois State University in the Special Education department. For my dissertation research, I am conducting a study about understanding the perceived impact of driver's education on young adults with visual impairments. The purpose of my study is to examine the role of driver's education in the lives of people with visual impairments, particularly how the course affects independent travel. [Family member] is a participant in this study and gave me your contact information. You have received this letter because you are related to a young adult with a visual impairment. I'm writing to invite you to participate in my study.

While you are under no obligation to participate in my study, I would very much value and appreciate your input. My hope is that this research will lead to improvements in transition experiences for people with visual impairments/blindness. If you decide to participate, I will schedule a single, 45-minute interview sometime between [DATE to be noted after approval] and June $1^{\text {st }}$.

Some of the risks associated with this study are that you may become shy at the thought of being recorded or uncomfortable sharing information you know is being recorded, or you may experience feelings of loss and sadness when talking about your child's experiences with driver's education and reliving a time when he or she was unable to share in the same milestone as his/her peers. If any of these occur, you are welcome to abstain from answering questions that cause undue emotional discomfort, you may take a break, or you may opt out of the research entirely. 
An additional risk is loss of confidentiality as your voice may be recognized on the digital audio recording. This risk will be minimized by the researcher using pseudonyms to protect your identity and other identifying information (town, family members). All voice recordings will also be kept in a locked filing cabinet that only the researcher has access to. While you will gain insight into what factors led to your family member traveling independently through vocalizing your experiences and having the opportunity to reflect upon them during the interview process, there is no direct benefit to you associated with participation in this research study.

If you would like to participate in this study or would like additional information, I'd be more than happy to speak with you and answer your questions. Feel free to contact me by replying to this email (․maclese@ilstu.edu) or calling me at 309-338-3716. Thank you for your time!

Sincerely, Molly Pasley, M.S.Ed

Illinois State University Doctoral Candidate 


\section{APPENDIX F: CONSENT LETTER FOR ADULT PARTICIPANTS WITH}

\section{VISUAL IMPAIRMENTS}

\section{Dear Participant:}

My name is Molly Pasley and I am a doctoral candidate in the Department of Special Education at Illinois State University. For my dissertation research, I am conducting a study about understanding the perceived impact of driver's education on young adults with visual impairments/blindness. The purpose of my study is to examine the role of driver's education in the lives of people with visual impairments, particularly how the course affects independent travel.

I am requesting your participation, which will involve a minimum of two individual interviews with me that will take place at a location convenient for you and last approximately 45 minutes. The interviews will be audio recorded with your permission and is a required component for participation. I will also observe you completing a daily activity. I will observe for one or more hours at a time. During observations, I will be writing down information about the ways in which you engage in independent travel. With your permission, I would also like to interview one or more of your family members. If you do not wish for me to speak with your family members, you can still participate in the study. All of these activities will take place between [DATE to be noted after approval] and June $1^{\text {st }}$.

Some of the risks associated with this study are that you may become shy at the thought of being recorded or uncomfortable sharing information you know is being recorded, or you may experience feelings of loss and sadness when talking about your experiences with driver's education and reliving a time when you were unable to share in the same milestone as your 
peers. If any of these occur, you are welcome to abstain from answering questions that cause undue emotional discomfort, you may take a break, or you may opt out of the research entirely.

An additional risk is loss of confidentiality as your voice may be recognized on the digital audio recording. This risk will be minimized by the researcher using pseudonyms to protect your identity and other identifying information (town, family members). All voice recordings will also be kept in a locked filing cabinet that only the researcher has access to.

The final risk to participants is undue influence or distress in feeling pressured to provide details for a family member to participate. Providing family member information is not required to participate in this study and you can choose to withdraw at any time.

While you will gain insight into what factors led to you traveling independently through vocalizing your experiences and having the opportunity to reflect upon them during the interview process, there is no direct benefit to you associated with participation in this research study.

Participation in this study is entirely voluntary. If you choose to participate in the study, you are free to withdraw permission at any time. You may also decline to participate. You will not be penalized for withdrawing or declining and all data collected up to the withdrawal date will be destroyed. The results of the research study will be published in my doctoral dissertation and may be published or presented, but your name will not be used. I will take all precautions to maintain confidentiality (your name will not be used, and the transcript from our interview will not be shared with anyone). For example, pseudonyms will be used during the interview and my doctoral dissertation.

If at any time during this study you have questions or concerns about this study your rights as a research subject, you may contact me at (309) 338-3716 or maclese@ilstu.edu. You may also contact Dr. Olaya Landa-Vialard at (309) 438-8986 or oalanda@ilstu.edu. 
Sincerely,

Molly Pasley, M.S.Ed

Illinois State University Doctoral Candidate

Please indicate your choice below by marking the statement and signing (or marking) your name.

Please sign both copies of this consent form and keep one copy.

I consent to participate in the study described above and have read this consent form.

I give you permission to contact my family member(s).

I do not give you permission to contact my family member(s).

Signature

Please print your name here

Date

(or verbal affirmation of participation marked by co-PI)

If you have any questions about your rights as a subject/participant in this research, or if you feel you have been placed at risk, you can contact the Research and Ethics \& Compliance Office at Illinois State University at (309) 438-2520 or Dr. Olaya Landa-Vialard at (309) 438-8986 or oalanda@ilstu.edu. 


\section{APPENDIX G: CONSENT LETTER FOR FAMILY MEMBER OF PERSON WITH}

\section{VISUAL IMPAIRMENTS}

\section{Dear Participant:}

My name is Molly Pasley and I am a doctoral candidate in the Department of Special Education at Illinois State University. For my dissertation research, I am conducting a study about understanding the perceived impact of driver's education on young adults with visual impairments/blindness. The purpose of my study is to examine the role of driver's education in the lives of people with visual impairments, particularly how the course affects independent travel.

[Family member] is a participant in this study and gave me your contact information. I am contacting you to gain richer insight into the experiences of young adults with visual impairments in terms of their independence. I am requesting your participation, which will involve a minimum of one individual interview with me that will take place at a location convenient for you and last approximately 45 minutes. The interviews will be audio recorded with your permission and is a required component for participation. Interviews will take place between [DATE to be noted after approval] and June $1^{\text {st }}$.

Some of the risks associated with this study are that you may become shy at the thought of being recorded or uncomfortable sharing information you know is being recorded, or you may experience feelings of loss and sadness when talking about your child's experiences with driver's education and reliving a time when he or she was unable to share in the same milestone as his/her peers. If any of these occur, you are welcome to abstain from answering questions that cause undue emotional discomfort, you may take a break, or you may opt out of the research entirely. 
An additional risk is loss of confidentiality as your voice may be recognized on the digital audio recording. This risk will be minimized by the researcher using pseudonyms to protect your identity and other identifying information (town, family members). All voice recordings will also be kept in a locked filing cabinet that only the researcher has access to.

While you will gain insight into what factors led to your family member traveling independently through vocalizing your experiences and having the opportunity to reflect upon them during the interview process, there is no direct benefit to you associated with participation in this research study.

Participation in this study is entirely voluntary. If you choose to participate in the study, you are free to withdraw permission at any time. You may also decline to participate. You will not be penalized for withdrawing or declining and all data collected up to the withdrawal date will be destroyed. The results of the research study will be published in my doctoral dissertation and may be published or presented, but your name will not be used. I will take all precautions to maintain confidentiality (your name will not be used, and the transcript from our interview will not be shared with anyone). For example, pseudonyms will be used during the interview and my doctoral dissertation.

If at any time during this study you have questions or concerns about this study your rights as a research subject, you may contact me at (309) 338-3716 or maclese@ilstu.edu. You may also contact Dr. Olaya Landa-Vialard at (309) 438-8986 or oalanda@ilstu.edu.

Sincerely, Molly Pasley, M.S.Ed

Illinois State University Doctoral Candidate 
Please indicate your choice below by marking the statement and signing (or marking) your name. Please sign both copies of this consent form and keep one copy.

I consent to participate in the study described above and have read this consent form.

I DO NOT consent to participate in the study described above.

\section{Signature \\ Please print your name here \\ Date}

(or verbal affirmation of participation marked by co-PI)

If you have any questions about your rights as a subject/participant in this research, or if you feel you have been placed at risk, you can contact the Research and Ethics \& Compliance Office at Illinois State University at (309) 438-2520 or Dr. Olaya Landa-Vialard at (309) 438-8986 or oalanda@ilstu.edu. 


\section{APPENDIX H: PARTICIPANT INTERVIEW PROTOCOL}

1. How is it that you came to be visually impaired?

2. Tell me about how you learned your independent travel skills.

a. Through school?

b. Through family?

If participants live independently, ask questions $3 \& 4$ :

3. I'd like to hear about your transition from living at home with your parents to living independently.

a. What changes did you have to make?

b. What issues came up that you didn't expect?

\section{How did your family react to this move away from home and to independent living?}

5. What are your worries about your own mobility or your ability to travel independently?

6. I'd like to hear about your experiences in driver's education.

a. How did this experience effect your self-confidence do you think?

b. What did it feel like being in this class?

c. What kinds of ways did it shape your independent travel?

7. If you were to give advice to other young people with visual impairments about taking driver's education, what would that be?

8. What would you say was the most important thing you learned from driver's education? 
9. In what kinds of ways do you feel driver's education has played into your independent travel now?

10. Is there anything else you'd like to share with me? 


\section{APPENDIX I: FAMILY MEMBER INTERVIEW PROTOCOL}

1. How is it that your [specific relationship to participant] came to be visually impaired?

a. How did you feel when you found out your family member had a visual impairment?

2. What kinds of things have you done, over time, to teach your [family member] about independent travel?

3. How do you feel about your [family member] traveling independently?

4. What kind of reactions have other family members had to [name's] learning independent travel?

5. How did [name's] schooling help with his or her independent travel?

If family member with a visual impairment lives independently, ask questions 6:

6. I'd like to hear about your experience of your family member's transition from living at home to living independently.

a. What changes did you make?

b. What issues came up that you didn't expect?

7. What are your worries about your family member's mobility and his/her ability to travel independently?

8. What kinds of changes have you seen in [name] now that [he or she] has more independent mobility skills?

9. Is there anything else you'd like to share with me? 